Instituto de Física de São Carlos - Universidade de São Paulo

Rafael Sola de Paula de Angelo Calsaverini

\title{
Acoplamento spin-órbita inter-subbanda em heteroestruturas semicondutoras
}


Rafael Sola de Paula de Angelo Calsaverini

\title{
Acoplamento spin-órbita inter-subbanda em heteroestruturas semicondutoras
}

\author{
Dissertação apresentada ao Instituto de \\ Física de São Carlos, da Universidade de São \\ Paulo, para obtenção do título de Mestre em \\ Ciências: Física Básica.
}

Orientador: José Carlos Egues

São Carlos 



\section{Agradecimentos}

Dedico essa dissertação às pessoas que nos legaram o patrimônio mais precioso que tinham: suas idéias. Na Filosofia, nas Artes, na Ciência, na Economia, na Política e na Religião, as grandes idéias animaram a História e influenciaram mais os rumos do mundo do que os grandes exércitos. Às grandes idéias! Às liberdades civis, ao Estado de direito ou à Teoria Atômica, à pintura barroca de Caravaggio ou ao contraponto de Bach, ao poema épico de Homero ou ao modernismo fantástico de Jorge Luis Borges. Não presumo que um dia eu possa alcançar o nível de excelência e genialidade dessas idéias, mas a elas dedico qualquer humilde e ingênua contribuição que eu possa fazer ao arcabouço intelectual da humanidade.

No plano pessoal, agradeço, é claro, a quem me trouxe à existência e a quem a proveu de momentos felizes. Agradeço a toda a minha enorme familia, que represento aqui por meus pais, José e Lourdes, e meus irmãos Carla, Rui, Túlio, minha falecida irmã Thayná, de quem me lembro sempre com carinho e devoção, e meu falecido irmão Thiago, a quem não conheci, mas nunca deixei de contar entre os irmãos.

À minha namorada Paola, à minha amiga e ex-companheira Priscilla, e aos amigos Clorô e Léo, as pessoas mais próximas de mim nos últimos anos, em cuja ausência eu não teria suportado passar por esse período difícil da minha vida pessoal.

Agradeço ao meu orientador, José Carlos Egues, pela paciência, tolerância e ao mesmo tempo pelo rigor e cobrança e pela oportunidade de trabalhar em uma pesquisa relevante. Ao professor Esmerindo Bernardes e aos colegas Gerson e Marco, agradeço pela colaboração e pela amizade. Pelo financiamento ao projeto agradeço ao CNPQ, pelos dois anos de bolsa, e também à CAPES que, através da Comissão de Pós Graduação do IFSC, financiou a compra de material computacional para o trabalho.

Aos moradores da República Asgard, Gerson (de novo?), Ivan, Alexandre, Dênis e Thi- 
ago, pelas palhaçadas constantes, pelos ovos podres em cima da geladeira, pelos momentos de troca de 'elogiosas' brincadeiras. Pelas gargalhadas essenciais à sanidade mental de qualquer pessoa.

Aos colegas das turmas de ingressantes do Bacharelado em Física no IFSC em 2000 e 2001, com quem convivi durante anos. Aos colegas que ainda estão na graduação do IFSC, com quem ainda dou boas risadas e discuto coisas sérias, eu peço: se esforcem para manter na Universidade a mesma racionalidade e seriedade que no passado defendemos e que agora parece novamente ameaçada. Que haja a percepção da necessidade de melhoras, mas que não se use o caos e a arbitrariedade como ferramenta.

Aos professores do IFSC, em particular aos professores Reginaldo Napolitano, Klaus Capelle, Liderio Ioriatti e Djalma Redondo, homens nos quais enxerguei qualidades que um dia pretendo ter.

Aos funcionários gentis, prestativos e eficientes do IFSC, que represento aqui através dos nomes: Edvane, Ana Mara e Beth, com cuja presteza e gentileza pude contar pelos quase 8 anos que passei por essa escola.

No que diz respeito ao plano espiritual, que é que existe aí? Há Deus? O numinoso, a imanência, o primeiro-motor? Que quer que ele seja (ou quem sabe ele não seja) sabe que eu anseio por conhecê-lo, sabe que lá me refugio e que, por maior que seja minha racionalidade e meu ceticismo, sempre tropeço e, até acidentalmente, caio de joelhos diante de algo que me transcende e que não posso apreender, mas que ao mesmo tempo está contido no mais profundo de mim mesmo. E diante desse numinoso e transcendente, não consigo ter outra atitude senão a de profunda reverência.

Sempre fui prolixo!! Não deveria ser diferente aqui, deveria?! Para terminar, eu não poderia nunca deixar de agradecer ao maior nome da música barroca alemã, Johann Gambolputty de von Ausfern Schplenden Schlitter Crasscrenbonfried Digger Dingle Dangledongledungle Burstein von Knackerthrasher Applebanger Horowitz Ticolensic Granderknotty-spelltinkle Grandlich Grumblemeyer Spelterwasser Kurstlich Himbleeisen Bahnwagen Gutenabend Bitteeinnürnburger Bratwustle Gerspurten Mitz-weimache Luberhundsfut Gumberaber Shönedanker Kalbsfleisch Mittler Aucher von Hautkopft of Ulm. 


\section{Alguma sabedoria...}

... para amolecer o coração dos examinadores e fingir erudição. Coisas que li durante a confecção dessa dissertação e que tiveram profundo impacto intelectual e emocional.

"Success is counted sweetest

By those who ne'er succeed.

To comprehend a nectar

Requires sorest need.

Not one of all the purple Host

Who took the Flag today

Can tell the definition

So clear of Victory

As he defeated-dying

On whose forbidden ear

The distant strains of triumph

Burst agonized and clear!"

Success - Emily Dickinson

"[...] Fui até ao campo com grandes propósitos.

Mas lá encontrei só ervas e árvores,

E quando havia gente era igual à outra.

Saio da janela, sento-me numa cadeira. Em que hei de pensar?

Que sei eu do que serei, eu que não sei o que sou?

Ser o que penso? Mas penso tanta coisa!

E há tantos que pensam ser a mesma coisa que não pode haver tantos!"

Tabacaria - Álvaro de Campos,

heterônimo de Fernando Pessoa

"Odi et amo. Quare id faciam fortasse requiris.

Nescio. Sed fieri sentio, et excrucior."

Carmina 85 - Gaius Valerius Catullus

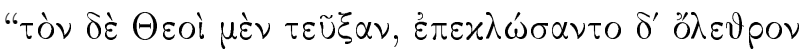

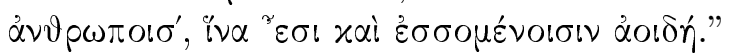

Odisséia de Homero, canto VIII, versos 579-580. 
AND NOW, FOR SOMETHING COMPLETELY DIFFERENT... A MASTER THESIS! 


\section{Resumo}

Neste trabalho apresentamos a determinação autoconsistente da constante de interação spin-órbita em heteroestruturas com duas sub-bandas. Como recentemente proposto [1, 2], ao obter o hamiltoneano de um sistema com duas sub-bandas na aproximação de massa efetiva, constata-se a presença de um acoplamento inter-subbanda que não se anula mesmo em heteroestruturas simétricas. Apresentamos aqui as deduções teóricas que levaram à proposição desse novo acoplamento e mostramos o cálculo autoconsistente da intensidade do acoplamento e a comparamos com a intensidade do acoplamento Rashba, já amplamente estudado. Discutimos o método $\vec{k} \cdot \vec{p}$ e a Aproximação da Função Envelope e mostramos a obtenção do modelo de Kane $8 \times 8$ para semicondutores com estrutura zincblende. Aplicamos o método do "folding down" ao hamiltoneano de Kane isolando o setor correspondente à banda de condução. Escrevemos dessa forma um hamiltoneano efetivo para a banda de condução no contexto de um poço quântico com uma barreira. Através da projeção desse hamiltoneano nos dois primeiros estados da parte orbital verifica-se o surgimento de um acoplamento inter-subbanda. Finalmente escrevemos o hamiltoneano efetivo $4 \times 4$ que descreve as duas primeiras subbandas de um poço quântico e obtivemos seus autoestados e autoenergias. Finalmente fizemos o cálculo autoconsistente das funções de onda e energias de um gás de elétrons em poços quânticos simples e duplos através da aproximação de Hartree e a partir desses resultados determinamos o valor da constante de acoplamento Rashba e da nova constante inter-subbanda. Entre os resultados obtidos destacam-se o controle elétrico da constante de acoplamento inter-subbanda através de um eletrodo externo e um efeito de renormalização da massa efetiva que pode chegar até $5 \%$ em algumas estruturas.

Palavras-Chave: Spintrônica, Acoplamento spin-órbita, Acoplamento inter-subbanda 


\section{Abstract}

In this work we present the self-consistent determination of the spin-orbit coupling constant in heterostructure with two subbands.As recently proposed [1,2], the effective hamiltonian for the conduction band in the effective mass approximation contains an intersubband spin-orbit coupling which is non-zero even for symmetric heterostructures. We present the theoretical derivation which leads to this proposal and show a selfconsistent determination of the coupling constant. We also compare the magnitude of the new coupling constant with the usual Rashba coupling. Starting with a discussion of the $\vec{k} \cdot \vec{p}$ method and the Envelope Function Approximation (EFA) we show the derivation of the $8 \times 8$ Kane model for semiconductors with zincblende structure. We then apply the "folding down" method, isolating the conduction band sector of the EFA hamiltonian. By projecting this hamiltonian in the first two states of the orbital part, we find an effective $4 \times 4$ hamiltonian that contains an inter-subband spin orbit coupling. The eingenvalues and eigenvectors of this hamiltonian are shown and, specializing the model for single and double quantum wells, we self-consistently determine the inter-subband and Rashba coupling constants in the Hartree approximation. The results indicate the possibility of electrical control of the coupling constant and show an effective mass renormalization effect that can be up to $5 \%$ in some cases.

KEYWORDS: Spintronics, Spin-orbit coupling, Inter-subband coupling 


\section{Lista de Figuras}

1 Exemplo de heteroestrutura semicondutora: um poço quântico de GaAs/ $\mathrm{Al}_{\mathrm{x}} \mathrm{Ga}_{1-\mathrm{x}} \mathrm{As}$.

Caso o confinamento seja suficientemente grande, na região do poço quântico há a formação de um gás de elétrons bidimensional (2DEG) com o "congelamento" do movimento dos elétrons na direção de crescimento da amostra. p. 14

2 Estrutura de bandas pictórica de um poço quântico evidênciando os perfis da banda de condução (em vermelho), bandas de buracos leves e pesados (em azul) e banda split-off (em verde) . . . . . . . . . . . . p. 15

3 Spin FET proposto por Datta e Das. Através do ajuste do potencial aplicado no gate pode-se controlar o valor da constante de acoplamento $\alpha$ e, portanto, o estado final do elétron ao atingir o dreno ferromagnético. Dessa forma é possível permitir ou não a passagem de corrente através do controle elétrico do spin. . . . . . . . . . . . . . . . . p. 20

4 GaAs - exemplo de rede de um semicondutor de estrutura zincblende. As esferas azuis representam átomos de gálio e as vermelhas átomos de arsênio. A rede típica do diamante po de ser obtida a partir dessa populando todos os vértices com átomos idênticos, por exemplo, C, Si ou Ge. . . . . . . . . . . . . . p. 29

5 Visão pictórica da formação de orbitais ligantes e antiligantes. Orbitais degenerados de átomos próximos combinam-se entre si. Combinações com alta densidade de carga entre os átomos possuem energia mais baixa e são chamadas orbitais ligantes, pois podem ser interpretadas como o surgimento de um potencial efetivo atrativo entre os íons. Combinações com baixa densidade de carga entre os átomos são denominadas anti-ligantes. . . . . . . . . . . . . p.30

6 Perfis estruturais e off-sets das bandas. Na equação (3.4), os perfis e os off-sets são usados para definir os potenciais efetivos nas diferentes bandas. p. 44 
7 Autoenergias da parte orbital em função de $k_{\text {॥ }}$ mostrando as sub-bandas contínuas $\quad$ p. 47

8 Um poço quântico simples e suas variáveis estruturais importantes: o tamanho do poço $L$ e a profundidade do potencial $V_{o} \ldots \ldots$. . . . . . . . . p. 55

9 Um exemplo de poço quântico duplo e variáveis estruturais relevantes. Além das já presentes no poço simples se incluem o a altura $V_{b}$ e largura $L_{b}$ da barreira. A altura $V_{b}$ pode ser maior ou menor do que a altura da barreira

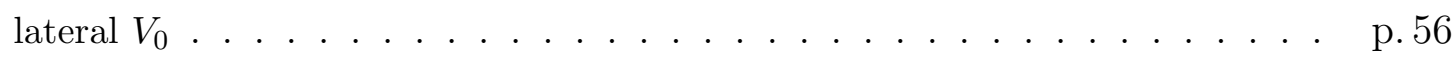

10 Estrutura de banda de um poço duplo mostrando os valores do gap e da energia split-off ao longo da estrutura.

11 Diagrama mostrando a interdependência das variáveis. Uma variável na base de uma seta é necessária para determinar a variável na ponta da mesma seta. A solução para esse problema é um método de aproximações sucessivas conhecido como Cálculo Autoconsistente . . . . . . . . . . . . . . . p. 64

12 Variação de $\eta$ com relação ao tamanho do poço para três poços quânticos duplos e um simples. A legenda indica a composição da barreira central. Nos casos $y=0.1$ e $y=0.2$ temos $V_{b}<V_{0}$, e no caso $y=0.3$ temos $V_{b}=V_{0}$. Para o caso sem barreira central temos um poço simples. Notase a diminuição em módulo da constante de acoplamento inter-subbanda conforme o confinamento é maior. . . . . . . . . . . . . . . p. 69

13 Contribuições a $\eta$ referentes à Fig. 12 devido aos diferentes termos. Notase que a escala de variação do termo oriundo do potencial de Hartree é bem menor do que a do termo estrutural. . . . . . . . . . . . . p p.70

14 Variação de $\eta$ com relação à densidade de portadores. A legenda indica a composição da barreira central e o tamanho do poço. Nota-se a dependência praticamente linear da constante inter-subbanda com relação à densidade areal de elétrons. O aumento de $\eta$ em módulo deve-se ao aumento da amplitude do potencial autoconsistente provocada pelo aumento da quantidade de carga no poço. . . . . . . . . . . . . . . p.71 
15 Contribuições a $\eta$ devido aos diferentes termos para os poços quânticos (duplos e simples) da Fig. 14. O código de cores é o mesmo utilizado naquela figura. Nota-se o comportamento aproximadamente linear da constante de acoplamento inter-subbanda em função da densidade. Notase ainda que a variação do termo associado ao potencial autoconsistente (Hartree+dopantes) é, desta vez, mais sensível que a do termo estrutural com relação à densidade de portadores. . . . . . . . . . . . . . . . p.72

16 Comparação da variação de $\eta$ e o preenchimento das subbandas. No gráfico superior observa-se $\eta$ vs. $n$. As linhas pontilhadas em azul e magenta são as melhores retas via regressão linear para os dois diferentes coeficientes angulares observados no gráfico. A linha vertical pontilhada indica a densidade para qual a segunda sub-banda começa a ser preenchida $\left(n=3.56 \times 10^{11} \mathrm{~cm}^{-2}\right)$. Na parte inferior observa-se a evolução da energia dos estados fundamental e $1^{\circ}$ excitado e também do potencial químico. O ponto que marca a mudança no coeficiente angular no gráfico superior é exatamente o ponto em que $\mu$ alcança a energia da segunda subbanda e esta começa a ser preenchida com elétrons. . . . . . . . . . . . . .

17 Variação de $\eta$ com relação aos parâmetros da barreira: altura e largura. Nota-se que o efeito do alargamento da barreira é tanto maior quanto maior a concentração de $\mathrm{Al}$ e vice-versa. O aumento da altura ou da largura da barreira implica em um aumento de $\eta_{\text {poço }}$ e $\eta_{\text {barreira }}$, contribuições de sinais diferentes. A competição entre essas duas contribuições determina a direção de variação de $\eta$. Nas situações simuladas, a variação de $\eta_{\text {barreira }}$ foi maior. . . . . . . . . . . . . . . . p. 75

18 Perfis de potencial e funções envelope para duas situações idênticas porém com barreiras de larguras diferentes. Nota-se que as amplitudes das funções envelope do estado fundamental e do primeiro excitado têm tendências diferentes. A combinação dessas duas variações em direções diferentes determinará a direção da variação de $\eta_{\text {barreira }} \ldots$. . . . . . . . . . . . p. 76 
19 Variação de $\eta$ com relação à concentração de alumínio no espaçador/altura do poço. A competição entre o aumento de $\eta_{\text {Hartree }}$ e a diminuição de $\eta_{\text {poço }}$ provoca a existência de um ligeiro mínimo em $\eta$. . . . . . . . . p.76

20 Gráficos de $\eta$ vs. altura da barreira central e densidade areal de portadores em poços de GaAs. Nota-se um comportamento similar aos dos poços de InSb, com uma diminuição em fatores de até ordem 100 no valor de $\eta$. . p.77

21 Descrição da posição dos eletrodos relativamente aos poços quânticos simulados. As voltagens aplicadas no front e noback gate foram variadas e foram determinadas a constante inter-subbanda e as constantes de Rashba associadas a cada sub-banda. . . . . . . . . . . . . . p. 78

22 Constantes de Acoplamento em um poço quântico duplo em função do potencial elétrico no front gate para um potencial de back gate de 100 $\mathrm{mV}$. Nota-se, como antes, um comportamento aproximadamente linear das constantes de acoplamento com relação ao potencial dos eletrodo. . p.79

23 Constantes de Acoplamento em um poço quântico simples em função do potencial elétrico no front gate para um potencial de back gate de $100 \mathrm{mV}$ para um poço com uma barreira central.Nota-se que a ordem de grandeza das constantes é similar, e parecidas com as das referências [3, 4]. Nota-se ainda o comportamento aproximadamente linear com relação ao potencial do eletrodo. . . . . . . . . . . . . . . . . . . . . . . . p. 79

24 Razão entre a diferença das massas efetivas nas subbandas e a massa efetiva do material para as simulações das Figs. 22 e 23. A diminuição em $\eta$ observada no gráfico contra a diferença de potencial leva a uma diminuição ainda mais acentuada na diferença entre as massas efetivas que é proporcional à $\eta^{2} \ldots \ldots \ldots$. . . . . . . . . . . . . . . . . . . 80 
25 Cálculo das constantes de acoplamento de Rashba e inter-subbanda e da razão entre as massas das diferentes subbandas para um poço quântico duplo sujeito a um potencial externo. A estrutura em questão não é neutra na situação simétrica e possui uma densidade de doadores na região dopada da ordem de 10 vezes maior do que as simuladas anteriormente graças e uma densidade de elétrons da mesma ordem de grandeza. Nota-se um efeito de renormalização de massa da ordem de $3 \%$ a $4 \%$ na situação simétrica e uma grande faixa de variação para as constantes de acoplamento em função da tensão do gate. . . . . . . . . . . . . . . . . p. p1 


\section{Lista de Tabelas}

1 Base de Estados $u_{m, \mathbf{0}}$ - Base de autoestados da matriz da equação (2.14) que será usada para calcular os elementos de matriz de Kane e as energias correspondentes no ponto $\Gamma \ldots \ldots$. . . . . . . . . . . . . . p. 32

2 Autoenergias e autofunções para o caso simétrico . . . . . . . . . p.50

3 Parâmetros de estrutura de bandas de ligas binárias III-V. . . . . . . . . p.58

4 Parâmetros de abaulamento e Valence Band Offset(VBO) para ligas ternárias p. 58

5 Parâmetros da simulação da figura $12 \ldots \ldots$. . . . . . . . . . . . 69

6 Parâmetros da simulação da Fig. 14 . . . . . . . . . . . . . . . . . . p.71 


\section{Sumário}

1 Introdução $\quad$ p. 13

1.1 Heteroestruturas semicondutoras . . . . . . . . . . . . p. 13

1.2 Acoplamento spin-órbita em semicondutores . . . . . . . . . . . p. 16

1.3 Exemplo de dispositivo spintrônico: Datta-Das Spin FET . . . . . . . . p. 18

1.4 Objeção de Ando . . . . . . . . . . . . . . . . . . p. 20

1.5 Referências Adicionais . . . . . . . . . . . . . . . p. 22

1.6 Objetivo do trabalho e outline . . . . . . . . . . . . p. 22

1.7 Resumo dos resultados . . . . . . . . . . . . . . . p. 23

2 O Método $\vec{k} \cdot \vec{p} \quad$ p. 25

2.1 Formulação . . . . . . . . . . . . . . . . . . . p. 25

2.2 Estrutura dos materiais de interesse e escolha da base . . . . . . . . . . p. 29

2.2.1 Considerações sobre a Estrutura Cristalina . . . . . . . . . . . p. 29

2.2.2 Efeito do Acoplamento Spin-Órbita sobre a base . . . . . . . . . p. 31

2.3 Matriz de Kane . . . . . . . . . . . . . . . . . . p. . . . . . . .

2.4 EFA - Aproximação da Função Envelope . . . . . . . . . . . . . . p. 35

2.5 Matriz de Kane em Heteroestruturas . . . . . . . . . . . . . p. 38

2.6 Aproximação de Hartree . . . . . . . . . . . . . . . . . . . . . . p. 39

3 Acoplamento Spin-Órbita Inter Sub-banda p. 42 
3.1 Introdução . . . . . . . . . . . . . . . . . . . . . . p. . 42

3.2 Folding Down . . . . . . . . . . . . . . . . . . p. 42

3.2 .1 Massa Efetiva . . . . . . . . . . . . . . . p. 45

3.3 Solução da parte orbital . . . . . . . . . . . . . . . . p. 46

3.4 Sistemas com duas sub-bandas . . . . . . . . . . . . . p. 47

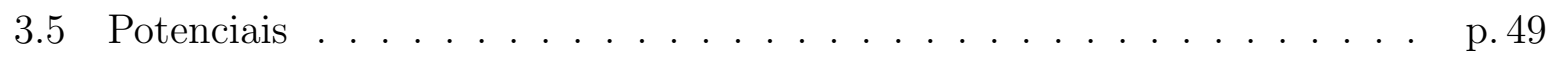

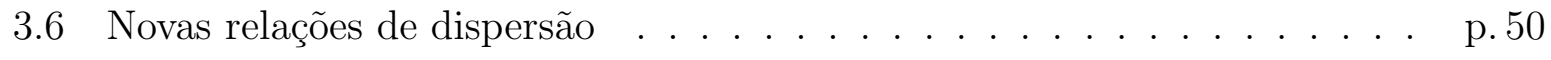

3.6.1 Autofunções e Autoenergias: estruturas simétricas . . . . . . . . . p. 50

3.6.2 Pequenos valores de $\eta$ : renormalização da massa . . . . . . . . p. p.51

3.6.3 Relação de dispersão na presença de assimetria . . . . . . . . . . p.51

4 Escolha dos Materiais e Estruturas p. 54

4.1 Sistemas a Serem Simulados . . . . . . . . . . . . . . p. . p4

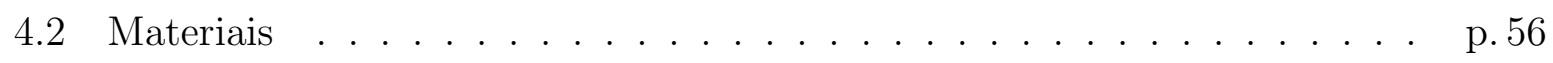

4.2.1 Parâmetros de Estrutura de Banda . . . . . . . . . . p. 57

5 Cálculo Autoconsistente e Aspectos Computacionais p. 60

5.1 Formulação do Problema . . . . . . . . . . . . . . . . . p. 60

5.1.1 Condições de Contorno na Equação de Poisson . . . . . . . . . . . p. 61

5.2 Potencial Químico e Densidade Eletrônica . . . . . . . . . . . . . p. 62

5.2 .1 Modelo de reservatório . . . . . . . . . . . . . p. 62

5.2.2 Modelo da Carga total constante . . . . . . . . . . . p. 62

5.3 Cálculo Autoconsistente . . . . . . . . . . . . . . p. 63

5.4 Métodos Numéricos . . . . . . . . . . . . . . . p. 64 
5.4.1 Equação de Schrödinger - Método de Numerov . . . . . . . . . . . p. 65

5.4.2 Equação de Poisson - Método de Numerov adaptado . . . . . . . p. 66

5.4 .3 Equação para o potencial químico . . . . . . . . . . . . p. 67

6 Resultados $\quad$ p. 68

6.1 Poços simétricos simples e duplos . . . . . . . . . . . . . p. 68

6.1.1 $\eta$ vs. tamanho do poço quântico . . . . . . . . . . . . p. 68

6.1.2 $\eta v s$. densidade bidimensional de portadores . . . . . . . . p. 70

6.1.3 $\eta$ vs. offset estrutural na banda de condução, largura da barreira e altura da barreira . . . . . . . . . . . . . . p. 74

6.1.4 Variando o Material do Poço . . . . . . . . . . . . . p. 77

6.2 Poços não simétricos e constante de Rashba . . . . . . . . . . . . . p.77

6.3 Massas efetivas . . . . . . . . . . . . . . . . p. 80

7 Conclusões $\quad$ p. 82

$\begin{array}{ll}\text { Referências } & \text { p. } 84\end{array}$

Apêndice A - Digressão sobre integrais de produtos de funções que $\begin{array}{lr}\text { variam em escalas diferentes } & \text { p. } 89\end{array}$

A.1 Cálculos dos elementos de matriz da Eq.(2.22) . . . . . . . . . . . p.91 


\section{Introdução}

\subsection{Heteroestruturas semicondutoras}

O desenvolvimento de técnicas para o crescimento de cristais de alto grau de pureza, deposição de camadas átomo a átomo, nanolitografia de precisão, microscopia com resolução atômica e criação de interfaces abruptas entre materiais revolucionou a tecnologia de dispositivos semicondutores. Poços quânticos, bicamadas e super-redes de alta mobilidade são hoje produzidas com extrema precisão e reprodutibilidade. Além dos benefícios tecnológicos, esses dispositivos permitiram acesso a uma grande gama de novos fenômenos físicos fundamentais, desde o Efeito Hall Quântico, medido em 1980 por von Klitzing[5] e o Efeito Hall Quântico Fracionário, encontrado por Tsui et al em 1982 [6], até recentes resultados como o Efeito Hall de Spin [7] e o Spin Coulomb Drag [8].

Uma heteroestrutura semicondutora é um arranjo espacial de diferentes materiais semicondutores. As diferenças na estrutura de bandas dos diferentes materiais permitem confinar elétrons em um movimento bidimensional (Two Dimensional Electron Gases 2DEG) e unidimensional (Quantum Wires) ou ainda mantê-los confinados nas 3 direções nos chamados pontos quânticos (Quantum Dots). Diversas outras geometrias podem ainda ser construídas através da deposição controlada de certos materiais e aplicação de diferenças de potencial controladas em eletrodos externos.

Na figura 1 temos um exemplo de heteroestrutura semicondutora típica: um poço quântico. Na figura, a composição da região chamada de "poço quântico" é indicada como sendo a liga semicondutora binária arseneto de gálio (GaAs), enquanto a composição região externa, chamada de "espaçador" é indicada como sendo a liga semicondutora ternária arseneto de gálio-alumínio $\left(\mathrm{Al}_{\mathrm{x}} \mathrm{Ga}_{1-\mathrm{x}} \mathrm{As}\right)$. Essa liga é obtida substituindo aleatóriamente 


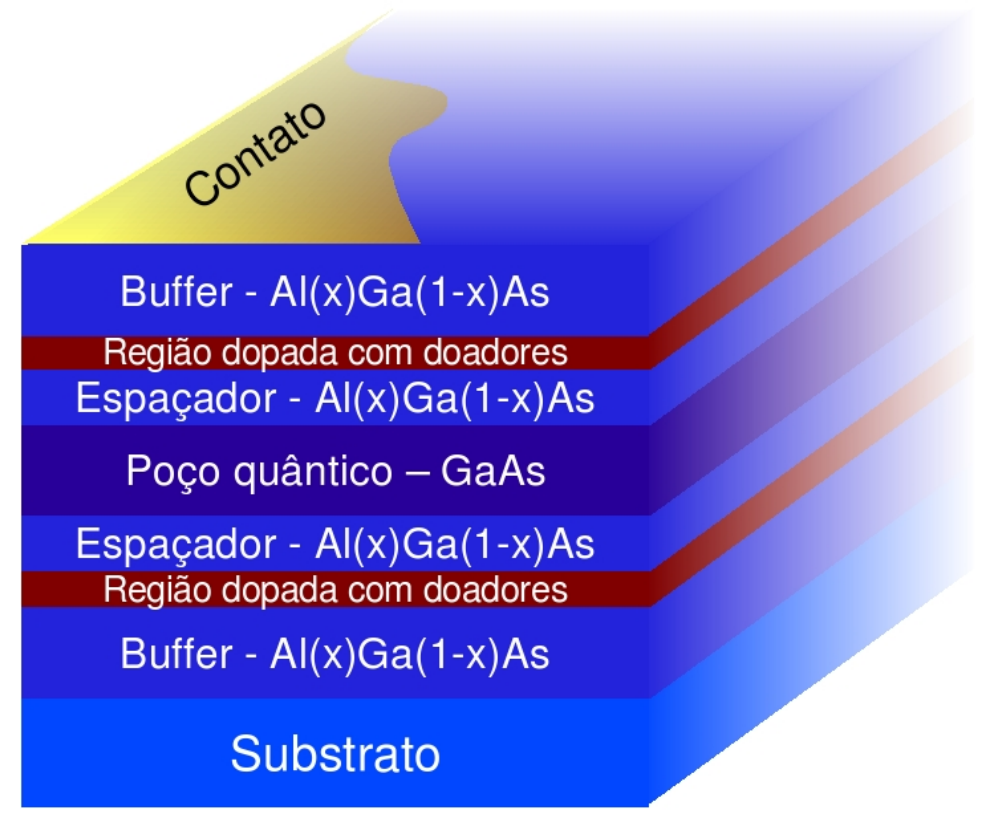

Figura 1: Exemplo de heteroestrutura semicondutora: um poço quântico de GaAs/ $\mathrm{Al}_{\mathrm{x}} \mathrm{Ga}_{1-\mathrm{x}} \mathrm{As}$. Caso o confinamento seja suficientemente grande, na região do poço quântico há a formação de um gás de elétrons bidimensional (2DEG) com o "congelamento" do movimento dos elétrons na direção de crescimento da amostra.

átomos de gálio $(\mathrm{Ga})$ por átomos de alumínio $(\mathrm{Al})$ de forma que a razão entre o número total de átomos de $\mathrm{Al}$ e o número total de cátions ( $\mathrm{Ga}$ ou $\mathrm{Al}$ ) na rede seja $x$. Ambos os elementos, Ga e Al, possuem camadas de valência terminadas nos orbitais $s^{2} p^{1}$, possuindo portanto propriedades químicas muito próximas. Ambos fornecem o mesmo número de elétrons para a ocupação das bandas de valência do cristal, o que implica que o material ainda é um semicondutor com a banda de valência cheia ${ }^{1}$. Como a substituição é aleatória, a periodicidade do cristal é quebrada. Entretanto é comum fazer uma aproximação conhecida como Aproximação do Cristal Virtual, em que o novo potencial atomístico não periódico é trocado por uma média ponderada na cela unitária entre os potenciais dos diferentes cátions usando as concentrações relativas como peso. O efeito da substituição é a alteração suave da estrutura de bandas do material e de fatores geométricos da rede - como o parâmetro de rede. No caso do $\mathrm{Al}_{\mathrm{x}} \mathrm{Ga}_{1-\mathrm{x}} \mathrm{As}$, o efeito da introdução do alumínio é o aumento do gap de energia no ponto $\Gamma$ e alteração da massa efetiva - tanto maior quanto maior a concentração $x$. O aumento do gap é distribuido entre as bandas de valência e condução em razões

\footnotetext{
${ }^{1}$ Não há introdução de portadores extra - sejam elétrons ou buracos.
} 


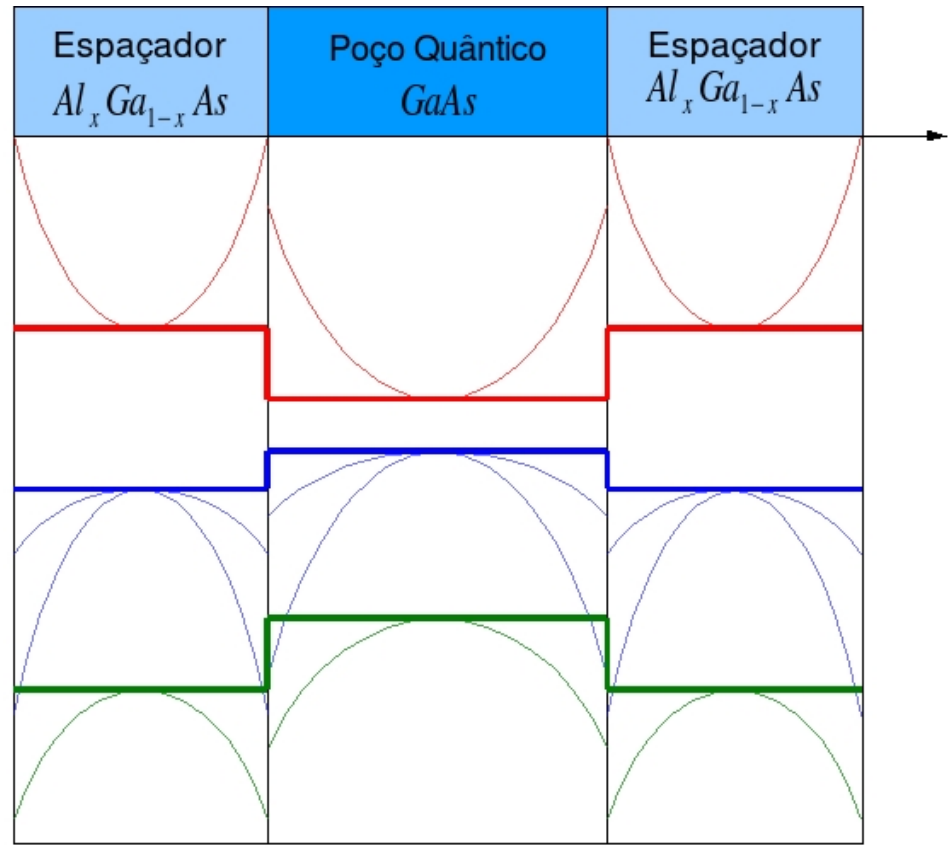

Figura 2: Estrutura de bandas pictórica de um poço quântico evidênciando os perfis da banda de condução (em vermelho), bandas de buracos leves e pesados (em azul) e banda split-off (em verde).

desiguais que variam para cada liga diferente. Em uma imagem pictórica, podemos dizer que se forma então, na heteroestrutura, uma estrutura de bandas dependente da posição, representada na figura 2. Para um elétron que eventualmente esteja no fundo da banda de condução, surge uma descontinuidade, um offset na energia do fundo da banda, que atua efetivamente como uma barreira de potencial, como mostrado em vermelho na figura 2 . Formam-se então níveis de energia confinados na direção de crescimento e livres no plano perpendicular à essa direção.

A região indicada em vermelho na figura 1 é uma região dopada com uma impureza substitucional doadora de elétrons. Pode-se substituir aleatóriamente átomos de gálio (ou alumínio) por átomos do grupo IV (silício, carbono, ...). Cada uma dessas impurezas tem suas camadas de valência terminadas em $s^{2} p^{2}$, fornecendo dessa forma um elétron extra com relação ao cátion original. O elétron extra passa então a popular a banda de condução tornando o material levemente condutor. Os elétrons fornecidos pelas impurezas doadoras se acumulam na região do poço, ocupando os estados quantizados na direção de crescimento. O movimento desses elétrons passa então a ser livre apenas no plano do poço, 
formando um gás de elétrons bidimensional (2DEG).

\subsection{Acoplamento spin-órbita em semicondutores}

A equação que rege o movimento dos elétrons em um sólido é a equação de Dirac, acrescida do potencial periódico associado aos íons da rede cristalina. O limite não relativístico dessa equação pode ser obtido, em potências de $\frac{E}{m c^{2}}$, onde $E$ é energia do elétron ${ }^{2}$. A primeira correção com relação à equação de Schrödinger para um elétron em um potencial $V(\vec{x})$ é o chamado acoplamento spin-órbita:

$$
H_{S O}=\frac{\hbar}{4 m^{2} c^{2}}[\vec{\sigma} \times \nabla V(\vec{x})] \cdot \vec{p}
$$

Sendo $\sigma_{x}$, sigma $a_{y}$ e $\sigma_{z}$ matrizes de Pauli. Em átomos esfericamente simétricos, o potencial depende apenas da distância ao núcleo $r$, de forma que o acoplamento spin-órbita se torna:

$$
H_{S O}=\frac{\hbar}{4 m^{2} c^{2}} \frac{\partial V}{\partial r}(\vec{\sigma} \times \hat{r}) \cdot \vec{p}=\frac{\hbar}{4 m^{2} c^{2}} \frac{1}{r} \frac{\partial V}{\partial r} \vec{\sigma} \cdot(\vec{r} \times \vec{p})=\frac{1}{2 m^{2} c^{2}} \frac{1}{r} \frac{\partial V}{\partial r} \vec{L} \cdot \vec{S} .
$$

Este é o famoso acoplamento $\vec{L} \cdot \vec{S}$, importante na determinação do espectro atômico. Uma visão pictórica da origem do acoplamento spin-órbita pode ser obtida através de uma mudança de referêncial - no referencial do elétron, o campo elétrico, proveniente do potencial a que o elétron está sujeito, transforma-se em um campo magnético que pode se acoplar com o spin do elétron através do acoplamento Zeeman. Esta dedução é imprecisa e ignora o fato de que o referencial do elétron é acelerado pois está em rotação. O tratamento adequado desse termo leva a um fator $\frac{1}{2}$ extra. ${ }^{3}$. Outra forma de deduzir corretamente o termo de interação spin-órbita é isolar no hamiltoniano de Dirac o setor correspondente às chamadas "componentes grandes" do spinor e realizar uma expansão em séries de $v^{2} / c^{2}[10$ $12]$.

Na determinação da estrutura de bandas de semicondutores o acoplamento spin-órbita pode se tornar importante. O efeito imediato é o de quebrar a degenerescência das bandas

\footnotetext{
${ }^{2}$ Em essência essa é uma expansão da equação de Dirac para velocidades muito baixas se comparadas com a velocidade da luz

${ }^{3} \mathrm{O}$ primeiro a deduzir a equação (1.1) corretamente a partir de uma mudança de referencial foi Llewellyn Thomas, em 1926, obtendo o fator $\frac{1}{2}$ originário do fenômeno que ficou conhecido como precessão de Thomas.[9]
} 
de valência mais altas no ponto $\Gamma$, gerando as bandas Split-off. Em redes cristalinas em que não há simetria de inversão, surge um termo na estrutura de bandas originário do termo spin-órbita denominado acoplamento de Dresselhaus[13, 14], cuja forma linearizada em poços quânticos é dada pela expressão:

$$
H_{D}=\beta\left(\sigma_{x} k_{x}-\sigma_{y} k_{y}\right)+\mathcal{O}\left(k^{3}\right)
$$

onde a constante $\beta$ depende de parâmetros do material e da geometria da estrutura. Este acoplamento é também conhecido como BIA - Bulk Inversion Asymmetry (assimetria de inversão em volume) - pois tem origem na ausência de simetria de inversão da rede cristalina e está presente mesmo em semicondutores na fase bulk ${ }^{4}$.

Em heteroestruturas pode se quebrar a simetria de inversão através de perfis assimétricos na modulação da concentração de dopantes, através de estruturas assimétricas (variação não simétrica da concentração de alumínio, heterojunções simples,...) ou através da aplicação de campos elétricos externos. Essa quebra da simetria de inversão dá origem a um termo conhecido como acoplamento spin-órbita de Rashba[15-18] ou SIA - Structural Inversion Asymmetry (assimetria estrutural de inversão). O termo associado a esse acoplamento é dado por $^{5,6}$ :

$$
H_{R}=\hbar \alpha \vec{\sigma} \cdot(\hat{z} \times \vec{k})
$$

onde a constante $\alpha$ depende do material e do grau de assimetria estrutural ${ }^{7}$. Através da aplicação de um campo elétrico na direção de crescimento pode-se aumentar ou diminuir essa assimetria, controlando assim o valor da constante $\alpha$ e a intensidade do acoplamento spin-órbita.

Recentemente foi proposto por Bernardes et al. $[1,2]$ um novo tipo de acoplamento spin-órbita em estruturas semicondutoras com mais de um estado confinado na direção de

\footnotetext{
${ }^{4}$ Segundo a nomenclatura difundida na área, a palavra bulk - do inglês, 'volume' - denota um material uniforme, de tamanho macroscópico, sem interfaces.

${ }^{5}$ Escolheu-se $\hat{z}$ como sendo a direção de crescimento do cristal.

${ }^{6}$ As matrizes $\sigma_{i}, i=x, y, z$, são as matrizes de Pauli descrevendo o grau de liberdade de spin.

${ }^{7}$ Uma expressão para $\alpha$ será apresentada no capítulo 3
} 
crescimento. O hamiltoniano associado a esse acoplamento inter-subbanda é dado por ${ }^{8}$ :

$$
H_{I}=\eta \tau^{x} \otimes \vec{\sigma} \cdot(\hat{z} \times \vec{k})
$$

A constante de acoplamento $\eta$ depende do material e de características da heteroestrutura. Esse novo acoplamento é similar ao de Rashba, porém não decorre de assimetria de inversão mas da diferença de paridade dos estados das diferentes sub-bandas. Portanto, a constante de acoplamento pode ser não-nula mesmo em heteroestruturas simétricas. Este termo encontrado acopla estados de spins opostos em diferentes subbandas e, apesar de não quebrar a degenerescência de spin, gera uma renormalização das massas efetivas nesses estados. Esse novo acoplamento também pode influenciar o Efeito Hall de Spin[19] e causar um Zitterbewegung ${ }^{9}$ não usual[1, 2]

\subsection{Exemplo de dispositivo spintrônico: Datta-Das Spin FET}

Uma importante proposta de dispositivo baseado na interação spin-órbita é o Spin Field Effect Transistor (transistor de efeito de campo de spin), proposto por Datta e Das [21]. Neste dispositivo, a possibilidade de se controlar a constante de acoplamento spin-órbita de Rashba permite realizar o controle elétrico do spin de um elétron.

O hamiltoniano de Rashba da Eq. (1.4), pode ser interpretado como um acoplamento do tipo Zeeman, com um campo magnético efetivo dependente de $\vec{k}$ :

$$
H_{R}=\frac{g}{2} \mu_{B} \vec{\sigma} \cdot \vec{B}(\vec{k})
$$

onde o campo efetivo $\vec{B}(\vec{k})$ é dado por

$$
\vec{B}(\vec{k})=\frac{2 \alpha \hbar}{g \mu_{B}}(\hat{z} \times \vec{k})
$$

Onde $g$ é o fator giromagnético, $m u_{B}$ é o magneton de Bohr e $\alpha$ a constante de acoplamento

\footnotetext{
${ }^{8}$ As matrizes $\tau_{i}, i=x, y, z$, são as matrizes de Pauli descrevendo o grau de liberdade de pseudo-spin associado à subbanda.

${ }^{9}$ Zitterbewegung é um movimento oscilatório previsto em mecânica quântica relativística para uma partícula de Dirac. Para mais detalhes ver [20].
} 
Rashba. Vamos considerar um elétron com velocidade na direção $\hat{x}^{10}$ inicialmente com spin up nesta direção, percorrendo uma distância $L$ em uma região com acoplamento spin órbita do tipo Rashba. O campo magnético efetivo é:

$$
\vec{B}(\vec{k})=\frac{2 \alpha \hbar k}{g \mu_{B}} \hat{y}
$$

O hamiltoniano da Eq.(1.6) será dado por:

$$
H_{R}=\alpha \hbar k \sigma_{y}
$$

O operador de evolução temporal associado ao hamiltoniano da Eq.(1.6) é11:

$$
U_{R}(t)=e^{-\frac{i H_{R} t}{\hbar}}=e^{-i \alpha k t \sigma_{y}}=\cos (\alpha k t) \mathcal{I}-i \sin (\alpha k t) \sigma_{y}
$$

No tempo t, o estado de spin do elétron será:

$$
|\psi(t)\rangle=U_{R}(t)|+\rangle_{x}=\cos (\alpha k t)|+\rangle+\sin (\alpha k t)|-\rangle
$$

Após um tempo $t=\frac{\pi}{2 \alpha k}$, o estado de spin será exatamente $|-\rangle$. Após um tempo $t=\frac{\pi}{\alpha k}$, o estado será exatamente $|+\rangle^{12}$. Uma vez que $L=v t=\frac{\hbar k}{m} t$, sendo $\mathrm{m}$ a massa do elétron no vácuo e $v$ a velocidade de propagação do elétron, escolhendo adequadamente o valor da constante $\alpha$ pode-se conseguir:

- Estado final $|+\rangle: \alpha=\frac{\pi \hbar}{2 m L}$.

- Estado final $|-\rangle: \alpha=\frac{\pi \hbar}{m L}$

Ao introduzir, como visto na Fig. 3, um emissor e um coletor ferromagnéticos com magnetização orientada em sentidos paralelos (ou antiparalelos), pode se injetar elétrons com uma certa direção de spin na região semicondutora e, dependendo do estado final do elétron, permitir ou não sua entrada no coletor. Uma vez que se pode controlar eletricamente o valor da constante de acoplamento $\alpha$, é possível controlar a passagem ou não de corrente através do controle de sua polarização de spin.

\footnotetext{
${ }^{10}$ Escolhemos $k_{y}=0$ e $k_{x}=k$

${ }^{11} \mathrm{O}$ símbolo $\mathcal{I}$ indica a matriz identidade.

${ }^{12}$ Ignorando um fator de fase global
} 


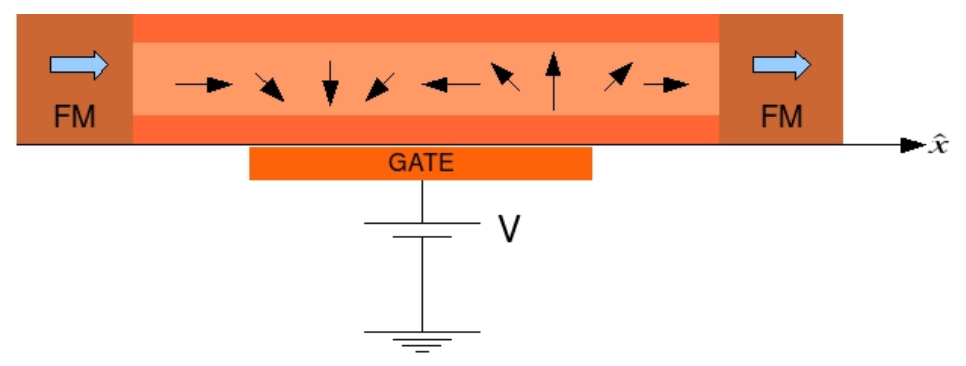

Figura 3: Spin FET proposto por Datta e Das. Através do ajuste do potencial aplicado no gate pode-se controlar o valor da constante de acoplamento $\alpha$ e, portanto, o estado final do elétron ao atingir o dreno ferromagnético. Dessa forma é possível permitir ou não a passagem de corrente através do controle elétrico do spin.

Até o momento não existe uma realização experimental do transístor Datta-Das. Existem dificuldades experimentais com a injeção de portadores polarizados, com os tempos de coerência de spin, com a detecção de portadores polarizados no dreno, a qualidade das interfaces, mecanismos de spin-flip, etc. Entretanto esse dispositivo continua motivando pesquisas experimentais e teóricas em spintrônica. Novas propostas modificadas de Spin-FET's foram feitas recentemente[22]. Diversos outros dispositivos spintrônicos para aplicação tecnológica e como sistemas modelo para pesquisa fundamental têm propostas e realizações continuamente reportadas na literatura. Pode-se citar por exemplo os Interferômetros de Spin [3] com realização experimental descrita em [23], beam-splitters (divisores de feixe) de elétrons com dependência de spin [24] que podem agir como detectores de correntes polarizadas, filtros de spin [25] e outros[26].

\subsection{Objeção de Ando}

Em 1976, Ando et al, apresentaram[27] uma objeção à existência de interação spinórbita na banda de condução. Baseando-se na dedução pictórica apresentada na seção 1.2, justifica-se a existência da interação spin-órbita em elétrons de condução através de uma mudança para o referêncial de repouso do elétron, em que ele deveria estar submetido a um campo magnético que se acopla com o spin do elétron através do hamiltoniano ${ }^{13}$ :

$$
H=\frac{\hbar}{4 m^{2} c^{2}}[\nabla V(\vec{x}) \times \vec{p}] \cdot \vec{\sigma}
$$

\footnotetext{
${ }^{13}$ Já incluindo o fator 2 proveniente da precessão de Thomas
} 
Onde $V(\vec{x})$ é o potencial estrutural causado pelos offsets nas bandas dos diferentes materiais que compõem uma heteroestrutura. No caso de um confinamento bidimensional, o potencial depende apenas de $z$ e, portanto:

$$
H=\frac{\hbar}{4 m^{2} c^{2}} \frac{\partial V(z)}{\partial z}[\hat{z} \times \vec{p}] \cdot \vec{\sigma}=\frac{\hbar}{4 m^{2} c^{2}} \frac{\partial V(z)}{\partial z}\left[p_{x} \sigma_{y}-p_{y} \sigma_{x}\right]
$$

A primeira correção em teoria de perturbação que esse termo faz na energia de um estado estacionário do poço quântico é obtida através do cálculo:

$$
\Delta E=\frac{\hbar^{2}}{4 m^{2} c^{2}}\left\langle\psi(z)\left|\frac{d V(z)}{d z}\right| \psi(z)\right\rangle\left[k_{x} \sigma_{y}-k_{y} \sigma_{x}\right]
$$

Onde $|\psi(z)\rangle$ é a parte orbital da função de onda na direção z. Agrupando as constantes ficamos com:

$$
\Delta E=\alpha\left[k_{x} \sigma_{y}-k_{y} \sigma_{x}\right]
$$

Essa correção têm a forma da interação de Rashba e a constante de acoplamento é dada por:

$$
\alpha=\frac{\hbar^{2}}{4 m^{2} c^{2}}\left\langle\psi(z)\left|\frac{d V(z)}{d z}\right| \psi(z)\right\rangle .
$$

Entretanto é um resultado da mecânica quântica básica que o valor médio da força $\left(\frac{d V(z)}{d z}\right)$ em um estado ligado é nulo. Esse problema foi tratado nas referências [28, 29], notando que em heteroestruturas a massa efetiva varia ao longo de z e que isso torna o valor médio de $\frac{d V(z)}{d z}$ não nulo. Entretanto a dedução acima apresentada do hamiltoniano de Rashba é imprecisa. No capítulo 3 faremos a dedução correta do hamiltoniano spin-órbita em heteroestruturas à partir da Aproximação da Função Envelope, isolando o setor correspondente à banda de condução através de um procedimento chamado Folding Down. Nessa dedução ficará claro que o potencial $\mathrm{V}(\mathrm{z})$ na equação 1.12 não é o perfil da banda de condução mas uma combinação dos perfis das bandas de valência. Dessa forma não há razão para supor que o valor médio da derivada desse potencial é nulo uma vez que não é o potencial que causa o confinamento[30, 31]. Essa discussão vale também para o acoplamento inter-subbanda: os campos elétricos importantes para os acoplamentos inter-subbanda e de Rashba são provenientes da banda de valência e não da banda de condução, além de eventuais potenciais externos aplicados através de eletrodos. 


\subsection{Referências Adicionais}

Na referência [32] há uma extensa introdução aos métodos de cálculo de estrutura de bandas e uma longa discussão da origem da interação spin-órbita. Uma outra dedução do hamiltoniano de Rashba é apresentada (Quasi-degenerate perturbation theory- teoria de perturbação quase-degenerada), equivalente às transformações de Foldy-Wouthuysen[11, 12] e ao método usado no presente trabalho, o Folding Down[1, 2]. O efeito da interação de Rashba leva a um movimento oscilatório similar ao Zitterbewegung de elétrons relativísticos [33]. O acoplamento spin-órbita inter-subbanda também apresenta um efeito similar segundo [1].As referências [17, 32, 34-38] fazem uso da abordagem $\vec{k} \cdot \vec{p}$ para o estudo da interação spin-órbita em semicondutores. A referência [39] estuda o acoplamento Rashba em nitretos com estrutura do tipo Wurtzita e apresenta o modelo da aproximação da função envelope nesses sistemas. A referência [40] trata dos efeitos do acoplamento de Rashba no espectro de minibandas de superredes assimétricas e [41] trata do efeito do acoplamento Rashba em quantum wires. As referências $[42,43]$ tratam do problema do controle da constante de Rashba através do controle do grau de assimetria das estruturas através de um campo externo e demonstram essa possibilidade experimentalmente medindo a constante de Rashba através de padrões de batimento nas oscilações de Schubnikov-de Haas. As referências $[44,45]$ tratam da medida da constante de Rashba através da Weak Antilocalization Analysis (análise de antilocalização fraca). A referência [28] discute o problema das forças elétricas médias em estados estacionários. Os autores mostram que diferenças nas massas efetivas nas diversas regiões podem levar a um valor não nulo do campo elétrico médio em estados ligados. O mesmo problema também é discutido num extenso artigo de revisão dos mesmos autores [29], que trata também de outros aspectos experimentais e teóricos. O cálculo autoconsistente de subbandas em heteroestruturas é descrito por [46-48].

\subsection{Objetivo do trabalho e outline}

Neste trabalho é feito um cálculo autoconsistente da nova constante de acoplamento spin-órbita inter-subbanda definida na equação (1.5) e da constante de Rashba (1.4) para 
uma série de heteroestruturas semicondutoras com duas subbandas. O cálculo é baseado no método $\vec{k} \cdot \vec{p}$ e na aproximação de Hartree para a interação entre os elétrons.

No capítulo 2 são introduzidos o método $\vec{k} \cdot \vec{p}$ e a Aproximação da Função Envelope na aproximação de 8 bandas (modelo de Kane), o modelo teórico que norteia todo o desenvolvimento posterior. No final do capítulo é introduzida a aproximação de Hartree. No capítulo 3 é feito o procedimento de folding down para encontrar o hamiltoniano efetivo que descreve a interação spin-órbita na banda de condução. As novas relações de dispersão são calculadas e é mostrado o efeito de renormalização da massa. No capítulo 4 é feita uma discussão sobre a escolha dos materiais e estruturas para o cálculo da constante de acoplamento inter-subbanda. No capítulo 5 discutimos sobre o cálculo autoconsistente dos níveis de energia e funções de onda de poços quânticos e sobre os métodos numéricos que foram utilizados nesse cálculo. No capítulo 6 são mostrados os resultados para o valor das constantes de acoplamento e como eles variam com relação a uma gama de parâmetros estruturais e físicos para poços quânticos duplos e simples.

\subsection{Resumo dos resultados}

Fizemos o cálculo da constante intersub-banda e da constante de Rashba para 2DEG confinados em poços quânticos duplos e simples, com e sem a aplicação de potenciais elétricos externos. Os cálculos foram realizados autoconsistentemente através da aproximação de Hartree e implementamos dois modelos para o cálculo do potencial químico do gás de elétrons: um modelo de reservatório e um modelo de densidade total constante. Dois tipos de poços foram simulados: poços duplos e poços simples.

O resultado principal desse trabalho se refere à intensidade relativa entre a constante de Rashba e a constante inter-subbanda: elas variam na mesma escala de grandeza, além do fato de que ambas podem ser controladas através da aplicação de potenciais elétricos externos. A presença da interação inter-subbanda gera um efeito de renormalização das massas efetivas das diferentes subbandas. Os valores obtidos para essa renormalização são pequenos, menores de que 1\%, para a maior parte das estruturas simuladas. Entretanto pode chegar até $5 \%$ em estruturas em que a diferença de energia entre os fundos das subbandas é pequena $(\approx 1 m e V)$. Variações dessa ordem de grandeza da massa efetiva 
podem ser observados em experimentos de ressonância de cíclotron. 


\section{O Método $\vec{k} \cdot \vec{p}$}

\subsection{Formulação}

O método $\vec{k} \cdot \vec{p}$ consiste em uma forma de adquirir informação sobre a estrutura de bandas de um material conhecendo informações apenas em um certo ponto do espaço recíproco $\overrightarrow{k_{0}}$. Esse método, quando aliado à Aproximação da Função Envelope (EFA - Envelope Function Approximation), torna possível obter, por exemplo, propriedades de heteroestruturas apenas com informações de propriedades do material em bulk. Essa abordagem permite também determinar o comportamento do material sob a ação de campos externos como campos elétricos e magnéticos. O objetivo desse capítulo é introduzir o Método $\vec{k} \cdot \vec{p}$ e a EFA e mostrar o modelo sobre o qual o presente trabalho foi desenvolvido.

Um elétron em um sólido cristalino está submetido ao potencial dos íons que formam uma rede cristalina periódica ${ }^{1}$. A equação que descreve o movimento de um elétron em um potencial eletrostático ${ }^{2} V(\vec{x})$ é a equação de $\operatorname{Dirac}^{3}$ :

$$
\left(\alpha_{0} m c^{2}+c \vec{\alpha} \cdot \vec{p}\right) \chi+V(\vec{x}) \chi=E \chi
$$

A função $\chi(\vec{x})$ é um espinor de quatro componentes e os símbolos $\alpha_{\nu}(\nu=0,1,2,3)$ são

\footnotetext{
${ }^{1}$ Ignorando-se possíveis defeitos e a agitação térmica dos íons, o que é uma excelente hipótese considerando experimentos feitos em baixas temperaturas e o nível de pureza alcançado em certos processos de preparação de heteroestruturas como a Epitaxia de Feixe Molecular. Pode-se ainda se valer da aproximação do cristal virtual mesmo na presença de impurezas, como no caso das ligas ternárias (AlGaAs e outras).

${ }^{2} \mathrm{Na}$ ausência de campos magnéticos

${ }^{3}$ Note que esta é a forma independente do tempo. Já foi feita a hipótese de que a função de onda é do tipo $e^{i E t / \hbar} \chi(\vec{x})$ pois o potencial $V(\vec{x})$ é independente do tempo.
} 
matrizes satisfazendo as relações de anti-comutação:

$$
\alpha_{\nu} \alpha_{\mu}+\alpha_{\mu} \alpha_{\nu}=0
$$

Para energias muito menores que a energia de repouso $m c^{2}$, uma expansão em série de potências da equação de Dirac na presença de um potencial $V(\vec{x})$ nos fornece uma equação similar à de Schrödinger com a adição de uma série de termos corretivos. Entre os termos de mais baixa ordem está o conhecido como acoplamento spin-órbita ${ }^{4}$ :

$$
\left[\frac{p^{2}}{2 m}+V(\vec{x})+\frac{\hbar}{4 m^{2} c^{2}}(\vec{\sigma} \times \nabla V) \cdot \vec{p}\right] \Psi_{n, \vec{k}}=H_{0} \Psi_{n, \vec{k}}=E_{n, \vec{k}} \Psi_{n, \vec{k}} .
$$

O potencial $V(\vec{x})$ é a soma do potencial devido aos íons da rede e à densidade de carga eletrônica. Uma vez que o potencial é periódico, o hamiltoniano assim definido $H_{0}$ é invariante por translações espaciais associadas a vetores da Rede de Bravais. Essa invariância resulta no chamado Teorema de Bloch ${ }^{5}$, um resultado central da física do estado sólido. Segundo esse teorema, as autofunções de $H_{0}$ podem ser escritas na forma:

$$
\Psi_{n, \vec{k}}(\vec{x})=e^{i \vec{k} \cdot \vec{x}} u_{n, \vec{k}}(\vec{x})
$$

em que as funções $u_{n, \vec{k}}(\vec{x})$ têm a mesma periodicidade do potencial $V(\vec{x})$. As funções de onda $\Psi_{n, \vec{k}}$ são conhecidas como funções de Bloch. Parte da importância desse resultado reside na periodicidade das funções $u_{n, \vec{k}}(\vec{x})$ - uma vez que elas são periódicas, basta que a equação de Schrödinger seja resolvida dentro de uma cela unitária com condições periódicas de contorno ${ }^{6}$ e não em todo o volume do cristal (que, em princípio, é infinito). Substituindo a Eq.(2.4) na equação de Schrödinger encontramos uma equação para as funções periódicas $u_{n, \vec{k}}\left(\right.$ definindo $\left.\epsilon_{n, \vec{k}}=E_{n, \vec{k}}-\frac{\hbar^{2} k^{2}}{2 m}\right)$ :

$$
\left[\frac{p^{2}}{2 m}+V(\vec{x})+\frac{\hbar}{4 m^{2} c^{2}}(\vec{\sigma} \times \nabla V) \cdot \vec{p}+\frac{\hbar}{m} \vec{k} \cdot\left(\vec{p}+\frac{\hbar}{4 m c^{2}} \vec{\sigma} \times \nabla V\right)\right] u_{n, \vec{k}}(\vec{x})=\epsilon_{n, \vec{k}} u_{n, \vec{k}}(\vec{x}) .
$$

Essa equação pode ser entendida como uma equação similar à equação de Schrödinger com

\footnotetext{
${ }^{4} \mathrm{O}$ termo de Darwin, a primeira correção relativística à energia cinética e outros termos estão sendo negligenciados. Uma derivação completa dessa equação pode ser vista em [12]

${ }^{5}$ Para uma definição de Rede de Bravais e uma prova do Teorema de Bloch procurar a referência [49]

${ }^{6} u_{n, \vec{k}}(\vec{x})=u_{n, \vec{k}}(\vec{x}+\vec{R})$ para todo vetor $\vec{R}$ pertencente à Rede Direta - ver referência [49].
} 
um hamiltoniano dependente de $\vec{k}$ dado por:

$$
H(\vec{k})=H_{0}+\frac{\hbar}{m} \vec{k} \cdot\left(\vec{p}+\frac{\hbar}{4 m c^{2}} \vec{\sigma} \times \nabla V\right)
$$

, sendo as funções $u_{n, \vec{k}}(\vec{x})$ suas autofunções ${ }^{7}$. Para explicitar a dependência em $\vec{k}$ pode-se reescrever a Eq.(2.5) na forma:

$$
\left[H_{0}+\frac{\hbar}{m} \vec{k} \cdot \vec{\pi}\right]|n, \vec{k}\rangle=\epsilon_{n, \vec{k}}|n, \vec{k}\rangle,
$$

em que o ket $|n, \vec{k}\rangle$ é tal que $\langle\vec{x} \mid n, \vec{k}\rangle=u_{n, \vec{k}}(\vec{x})$ e o vetor $\vec{\pi}$ é uma abreviação para $\vec{p}+$ $\frac{\hbar}{4 m c^{2}} \vec{\sigma} \times \nabla V$. Podemos encarar o hamiltoniano $H(\vec{k})$ como a soma do operador $H_{0}$ e uma perturbação proporcional a $\vec{k}$.

As funções $u_{n, \vec{k}}$ para um certo $\vec{k}$ são o conjunto das autofunções do operador hermitiano $H(\vec{k})$ com condições de contorno periódicas na cela unitária. Portanto, para um certo va lor do vetor de Bloch, digamos $\vec{k}_{0}$, o conjunto das funções $u_{n, \vec{k}_{0}}$ é completo no espaço vetorial das funções periódicas na cela unitária. Dessa forma, podemos expandir qualquer dos autovetores $|n, \vec{k}\rangle$ em uma combinação linear dos autovetores $\left|l, \vec{k}_{0}\right\rangle$,

$$
|n, \vec{k}\rangle=\sum_{l} C_{l}\left(\vec{k}, \vec{k}_{0}\right)\left|l, \vec{k}_{0}\right\rangle
$$

Os materiais que interessam para o presente trabalho são semicondutores de gap direto ${ }^{8}$. Assim, faremos a expansão acima em torno do ponto $\Gamma$, ou seja, o ponto $\vec{k}_{0}=\mathbf{0}$. Denotando por $|l\rangle$ o autovetor $|l, \mathbf{0}\rangle$, temos:

$$
|n, \vec{k}\rangle=\sum_{l} C_{l}(\vec{k}, \mathbf{0})|l\rangle=\sum_{l} C_{l}(\vec{k})|l\rangle
$$

Note que os estados $|l\rangle$ satisfazem a Eq.(2.6) com $\vec{k}=\mathbf{0}$ :

$$
\left[\frac{p^{2}}{2 m}+V(\vec{x})+\frac{\hbar}{4 m^{2} c^{2}}(\vec{\sigma} \times \nabla V) \cdot \vec{p}\right]|l\rangle=E_{l}^{0}|l\rangle .
$$

O estado $|l\rangle$ é, portanto, autovetor de $H_{0}$ e o seu autovalor $E_{l}^{0}=E_{l, \mathbf{0}}$. O próximo passo é

\footnotetext{
${ }^{7} \mathrm{Na}$ ausência de interação spin-órbita, o termo proporcional a $\vec{k}$ em $H(\vec{k})$ torna-se $\frac{\hbar}{m} \vec{k} \cdot \vec{p}$, derivando-se daí o nome do método.

${ }^{8}$ Materiais em que o fundo da banda de condução encontra-se no ponto $\Gamma$, alinhado com o topo da banda de valência.
} 
substituir na Eq.(2.6) a expansão feita na Eq.(2.7):

$$
\begin{aligned}
{\left[H_{0}+\frac{\hbar}{m} \vec{k} \cdot \vec{\pi}\right]|n, \vec{k}\rangle } & =\epsilon_{n, \vec{k}}|n, \vec{k}\rangle, \\
{\left[H_{0}+\frac{\hbar}{m} \vec{k} \cdot \vec{\pi}\right] \sum_{l} C_{l}(\vec{k})|l\rangle } & =\epsilon_{n, \vec{k}} \sum_{l} C_{l}(\vec{k})|l\rangle, \\
\sum_{l} C_{l}(\vec{k})\left[E_{l}^{0}+\frac{\hbar}{m} \vec{k} \cdot \vec{\pi}\right]|l\rangle & =\epsilon_{n, \vec{k}} \sum_{l} C_{l}(\vec{k})|l\rangle .
\end{aligned}
$$

Multiplicando a Eq.(2.9) pelo bra $\langle s|$ temos:

$$
\left\langle s\left|\sum_{l} C_{l}(\vec{k})\left[E_{l}^{0}+\frac{\hbar}{m} \vec{k} \cdot \vec{\pi}\right]\right| l\right\rangle=\left\langle s\left|\epsilon_{n, \vec{k}} \sum_{l} C_{l}(\vec{k})\right| l\right\rangle .
$$

O que, finalmente, leva a:

$$
\left(E_{s}^{0}-\epsilon_{n, \vec{k}}\right) C_{s}(\vec{k})+\frac{\hbar}{m} \vec{k} \cdot \sum_{l}\langle s|\vec{\pi}| l\rangle C_{l}(\vec{k})=0
$$

A Eq.(2.10) pode ser colocada na forma de uma equação matricial:

$$
\left(\begin{array}{ccc}
\left(E_{1}^{0}-\epsilon_{n, \vec{k}}\right)+\frac{\hbar}{m} \vec{k} \cdot \vec{\pi}_{11} & \frac{\hbar}{m} \vec{k} \cdot \vec{\pi}_{12} & \ldots \\
\frac{\hbar}{m} \vec{k} \cdot \vec{\pi}_{21} & \left(E_{2}^{0}-\epsilon_{n, \vec{k}}\right)+\frac{\hbar}{m} \vec{k} \cdot \vec{\pi}_{22} & \ldots \\
\vdots & \vdots & \ddots
\end{array}\right)\left(\begin{array}{c}
C_{1}(\vec{k}) \\
C_{2}(\vec{k}) \\
\vdots
\end{array}\right)=0 .
$$

Resolvendo esse problema de autovalores, as energias $\epsilon_{n, \vec{k}}$ são obtidas em função de parâmetros do material que só dependem do ponto $\vec{k}=0$ : os elementos de matriz $\vec{\pi}_{m n}$ e das energias $E_{l}^{0}$. Esses parâmetros podem ser obtidos a partir de experimentos, tornando o método $\vec{k} \cdot \vec{p}$ uma abordagem semi-empírica. 


\subsection{Estrutura dos materiais de interesse e escolha da base}

\subsubsection{Considerações sobre a Estrutura Cristalina}

Os materiais que serão tratados no presente trabalho são semicondutores do grupo III $-V^{9}$ com estrutura cristalina zincblende, exemplificada na Fig.4. Nessa estrutura, os átomos possuem em suas camadas de valência orbitais do tipo $s$ e do tipo $p$ que se hibridizam em ligações tetragonais. Assim, cada átomo liga-se a quatro vizinhos. Conforme os

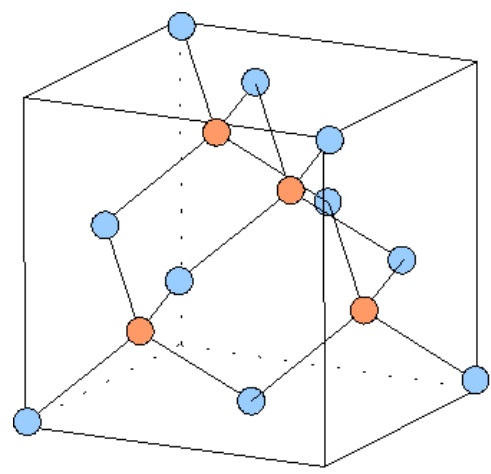

Figura 4: GaAs - exemplo de rede de um semicondutor de estrutura zincblende. As esferas azuis representam átomos de gálio e as vermelhas átomos de arsênio. A rede típica do diamante po de ser obtida a partir dessa populando todos os vértices com átomos idênticos, por exemplo, $\mathrm{C}, \mathrm{Si}$ ou Ge.

átomos se aproximam para formar a rede cristalina, combinam-se de orbitais de diferentes átomos para que se formem as ligações químicas. Os orbitais híbridos $s p^{3}$ de átomos vizinhos se combinam para formar orbitais moleculares ligantes e anti-ligantes, como se vê na Fig.5. No limite em que os átomos se arranjam em uma rede infinita, cada um desses estados dá origem a uma banda de energias de valência (estado ligante) e de condução (estado anti-ligante). Entre a banda de valência mais energética (tipo $p$ ) e a banda de condução menos energética (tipo $s$ ), forma-se um gap de energias não permitidas. Esses estados acoplam-se através dos elementos de matriz $\vec{\pi}_{m n}$, como se pode ver na Eq.2.11. Esses elementos são, ignorando por um momento o termo que surge da interação spin-órbita, elementos de matriz do operador momentum das funções de Bloch no ponto $\Gamma$. Consi-

\footnotetext{
${ }^{9} \mathrm{O}$ modelo apresentado também é válido para semicondutores II-VI com estrutura zincblende
} 


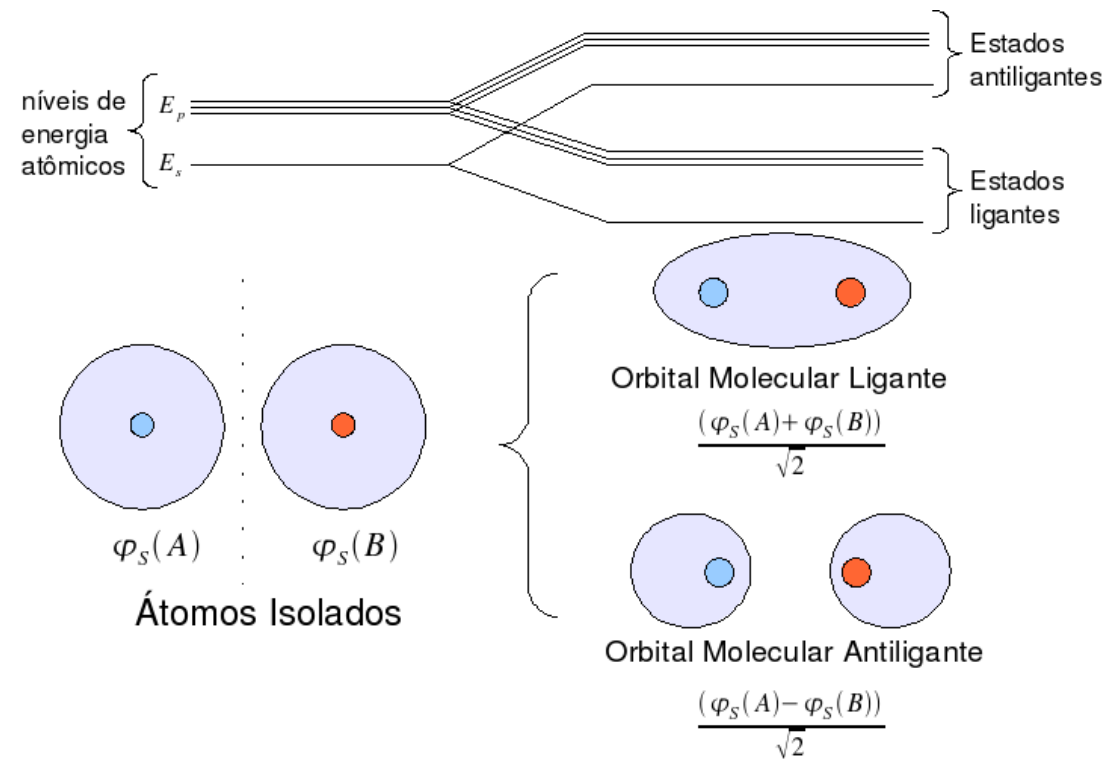

Figura 5: Visão pictórica da formação de orbitais ligantes e antiligantes. Orbitais degenerados de átomos próximos combinam-se entre si. Combinações com alta densidade de carga entre os átomos possuem energia mais baixa e são chamadas orbitais ligantes, pois podem ser interpretadas como o surgimento de um potencial efetivo atrativo entre os íons. Combinações com baixa densidade de carga entre os átomos são denominadas anti-ligantes.

derações de simetria mostram ${ }^{10}$ que esses elementos acoplam estados com simetria do tipo $\mathrm{s}$ apenas com estados com simetria do tipo p. O acoplamento mais relevante desse tipo é o dos estados que darão origem à banda de condução em questão com os que formarão as bandas de valência mais altas. Por essa razão, para o que se segue, será mantido na matriz da Eq.(2.11) apenas o bloco 8x8 contendo os estados que darão origem à banda de condução e às três bandas de valência mais altas, representados pela base:

$$
|s\rangle| \pm\rangle \quad\left|p_{i}\right\rangle| \pm\rangle
$$

com $i=x, y, z$. Esse é o chamado modelo de Kane para estrutura de bandas.

\footnotetext{
${ }^{10}$ Essa discussão pode ser encontrada nas seções 2.6 e 2.7 da referência [50]
} 


\subsubsection{Efeito do Acoplamento Spin-Órbita sobre a base}

A base de estados para calcular os elementos de matriz $\vec{\pi}_{m n}$ deve ser, como mostra a Eq.(2.8), uma base de autoestados do operador ${ }^{11}$ :

$$
H(\vec{k}=\mathbf{0})=\frac{p^{2}}{2 m}+V(\vec{x})+\frac{\hbar}{4 m^{2} c^{2}}(\vec{\sigma} \times \nabla V) \cdot \vec{p}
$$

Esse operador possui um termo associado ao movimento orbital:

$$
H_{o r b}=\frac{p^{2}}{2 m}+V(\vec{x})
$$

e um temo associado à interação spin-órbita:

$$
H_{S O}=\frac{\hbar}{4 m^{2} c^{2}}(\vec{\sigma} \times \nabla V) \cdot \vec{p}
$$

Os estados da Eq.(2.12) são autoestados da parte orbital apenas. Os estados tipo $s$ são autoestados de $H_{\text {orb }}$ com certo autovalor $E_{s}$ e os do tipo $p$ são todos degenerados e têm certo autovalor $E_{p}<E_{s}$. Podemos usar esses estados para encontrar uma forma matricial para o operador da Eq.(2.13) e diagonalizá-lo. Novamente argumentos de simetria mostram que os únicos elementos de matriz não nulos de $H_{S O}$ são aqueles que contêm os termos:

$$
\frac{3 \hbar^{2}}{4 c^{2} m^{2}}\left\langle p_{x}\left|\frac{\partial V}{\partial y} \frac{\partial}{\partial x}-\frac{\partial V}{\partial x} \frac{\partial}{\partial y}\right| p_{y}\right\rangle \equiv \Delta
$$

e similares. O parâmetro $\Delta$ é denominado energia de desdobramento spin-órbita, ou energia de split-off. A matriz que representa o operador na Eq.(2.13) fica, portanto, com a seguinte forma bloco diagonal:

\footnotetext{
${ }^{11}$ Auto-funções periódicas dentro da cela primitiva
} 


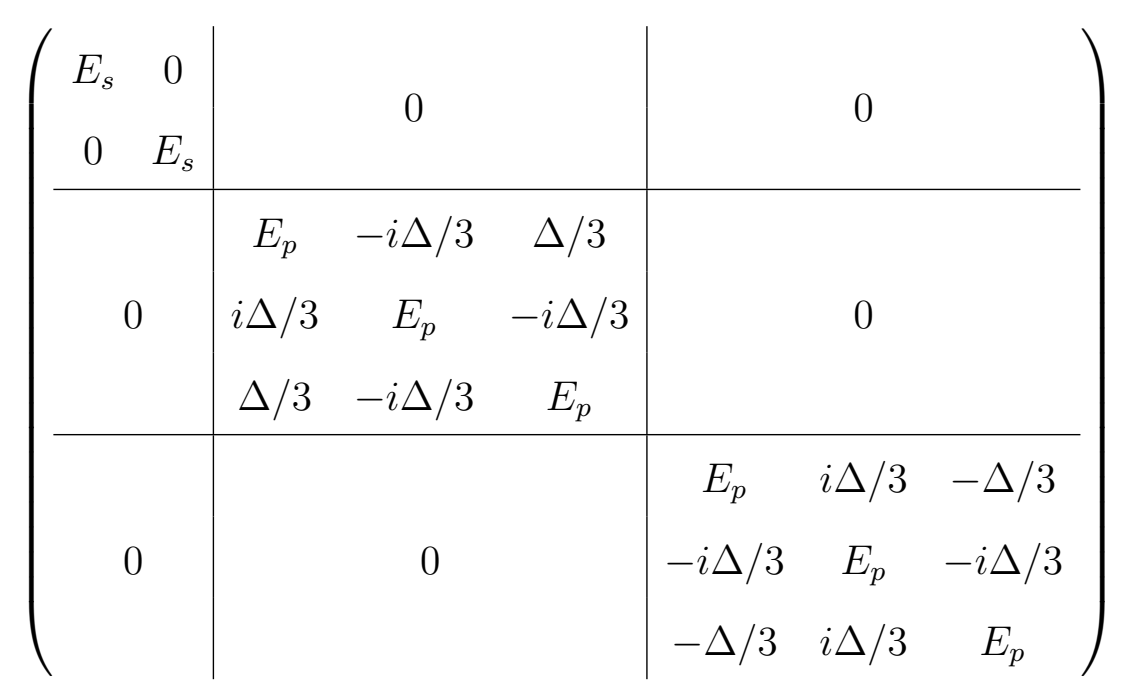

Diagonalizando essa matriz, encontramos a base de autovetores com as respectivas energias para $\vec{k}=0$, apresentados na tabela 1 . Note que as fases escolhidas são as mesmas da referência [51], para que os elementos de matriz sejam todos reais. Podemos redefinir essas

Tabela 1: Base de Estados $u_{m, \mathbf{0}}$ - Base de autoestados da matriz da equação (2.14) que será usada para calcular os elementos de matriz de Kane e as energias correspondentes no ponto $\Gamma$.

\begin{tabular}{|c|c|c|}
\hline & Energ & \\
\hline \multicolumn{3}{|c|}{ Estados com $l=0, \sigma=\frac{1}{2} \Longrightarrow j=l+\sigma=\frac{1}{2}$} \\
\hline$|1\rangle=i|s\rangle|+\rangle$ & $E_{1}^{0}=E_{s}$ & $\left|\frac{1}{2}, \frac{1}{2}\right\rangle$ \\
\hline$|2\rangle=i|s\rangle|-\rangle$ & $E_{2}^{0}=E_{s}$ & \\
\hline \multicolumn{3}{|c|}{ Estados com $l=1, \sigma=\frac{1}{2} \Longrightarrow j=l+\sigma=\frac{3}{2}$} \\
\hline$|3\rangle=\frac{1}{\sqrt{2}}(|x\rangle-i|y\rangle)|+\rangle$ & $E_{3}^{0}=E_{p}+\frac{\Delta}{3}$ & $\left|\frac{3}{2}, \frac{3}{2}\right\rangle$ \\
\hline$|4\rangle=-\frac{1}{\sqrt{6}}(|x\rangle+i|y\rangle)|-\rangle+\sqrt{\frac{2}{3}}|z\rangle|+\rangle$ & $E_{4}^{0}=E_{p}+\frac{\Delta}{3}$ & $\left|\frac{3}{2}, \frac{1}{2}\right\rangle$ \\
\hline$|5\rangle=\frac{1}{\sqrt{6}}(|x\rangle-i|y\rangle)|+\rangle+\sqrt{\frac{2}{3}}|z\rangle|-\rangle$ & $E_{5}^{0}=E_{p}+\frac{\Delta}{3}$ & $\left|\frac{3}{2},-\frac{1}{2}\right\rangle$ \\
\hline$|6\rangle=-\frac{1}{\sqrt{2}}(|x\rangle+i|y\rangle)|-\rangle$ & $E_{6}^{0}=E_{p}+\frac{\Delta}{3}$ & $\left|\frac{3}{2},-\frac{3}{2}\right\rangle$ \\
\hline \multicolumn{3}{|c|}{ Estados com $l=1, \sigma=\frac{1}{2} \Longrightarrow j=l-\sigma=\frac{1}{2}$} \\
\hline$|7\rangle=-\frac{1}{\sqrt{3}}(|x\rangle+i|y\rangle)|-\rangle-\frac{1}{\sqrt{3}}|z\rangle|+\rangle$ & $E_{7}^{0}=E_{p}-\frac{2 \Delta}{3}$ & $\left|\frac{1}{2}, \frac{1}{2}\right\rangle$ \\
\hline$|8\rangle=-\frac{1}{\sqrt{3}}(|x\rangle-i|y\rangle)|+\rangle+\frac{1}{\sqrt{3}}|z\rangle|-\rangle$ & $E_{8}^{0}=E_{p}-\frac{2 \Delta}{3}$ & $\left|\frac{1}{2},-\frac{1}{2}\right\rangle$ \\
\hline
\end{tabular}


energias da seguinte forma:

$$
\begin{aligned}
E_{p}+\frac{\Delta}{3} & =E_{s}-E_{g} \\
E_{p}-\frac{2 \Delta}{3} & =E_{s}-E_{g}-\Delta
\end{aligned}
$$

Com $E_{g}=E_{s}-E_{p}-\frac{\Delta}{3}$. Assim, ficamos com três grupos de estados degenerados em $\vec{k}=0$ : dois com energia $E_{s}$, quatro com energia $E_{s}-E_{g}$ e dois com energia $E_{s}-E g-\Delta$. Esses estados descritos acima nada mais são que autoestados dos operadores $j^{2}$ e $j_{z}$, sendo $\vec{j}=\frac{\hbar}{2} \vec{\sigma}+\vec{L}$ o operador momento angular total. Os autovetores de $j^{2}$ e $j_{z}$ para a soma de um momento angular $l=1$ e um spin $s=1 / 2$ estão explicitamente calculados na referência [52] ${ }^{12}$. Ilustrativamente vamos calcular a ação de $j_{z}$ sobre o estado $|3\rangle$. Note que, na notação que usamos aqui:

$$
\begin{aligned}
& \left|p_{x}\right\rangle=-\frac{1}{\sqrt{2}}(|l=1, m=1\rangle-|l=1, m=-1\rangle) \\
& \left|p_{y}\right\rangle=i \frac{1}{\sqrt{2}}(|l=1, m=1\rangle+|l=1, m=-1\rangle)
\end{aligned}
$$

Logo:

$$
|3\rangle=|l=1, m=1\rangle|+\rangle
$$

Aplicando o operador $j_{z}=l_{z}+\frac{1}{2} \sigma_{z}$ sobre o estado $|3\rangle$ :

$$
j_{z}|3\rangle=\left(l_{z}|l=1, m=1\rangle\right)|+\rangle+\frac{1}{2}|l=1, m=1\rangle \sigma_{z}|+\rangle=(1)|3\rangle+\left(\frac{1}{2}\right)|3\rangle=\frac{3}{2}|3\rangle
$$

Na terceira coluna da tabela 1 mostramos os estados classificados de acordo com números quânticos $j$ e $m$.

\subsection{Matriz de Kane}

De posse da base definida na tabela 1 , pode-se calcular os elementos de matriz $\vec{\pi}_{n m}$ e determinar as energias para $\vec{k} \neq 0$. Esses elementos de matriz envolvem duas partes: o elemento de matriz do operador momentum $\left(\vec{p}_{n m}=\langle n|\vec{p}| m\rangle\right)$ e o elemento de matriz do termo oriundo do acoplamento spin-órbita $\left(\left\langle n\left|\frac{\hbar}{4 m^{2} c^{2}} \vec{\sigma} \times \nabla V\right| m\right\rangle\right)$. A interação spin-

\footnotetext{
${ }^{12}$ capítulo $\mathrm{X}$ do volume II, complemento $A_{X}$
} 
órbita já foi incluída em primeira aproximação nesse cálculo através da energia de split-off. Desprezaremos, assim, por simplicidade, o segundo termo mantendo apenas.

$$
\vec{\pi}_{m n}=\vec{p}_{m n}
$$

Os únicos elementos de matriz não nulos serão aqueles que contém termos do tipo $\left\langle s\left|p_{k}\right| x_{k}\right\rangle$, com $x_{k}=x, y, z$. Esses termos têm todos o mesmo valor, que denotaremos por:

$$
P=-\frac{-i \hbar}{m}\left\langle s\left|p_{x}\right| x\right\rangle
$$

P é chamado elemento de matriz de Kane, que é uma quantidade real. Com essa definição, os elementos de matriz podem ser todos calculados e a matriz da Eq.(2.11) pode ser escrita de forma explícita. A matriz final se divide em blocos, sendo dada por:

$$
\left(\begin{array}{cc}
H_{c} & W_{c v} \\
W_{c v} & H_{v}
\end{array}\right)
$$

Onde os blocos $H_{c}$ e $H_{v}$ são diagonais e dados por:

$$
\begin{aligned}
& H_{c}=E_{s} \mathcal{I}_{2 \times 2} \\
& H_{v}=\left(\begin{array}{cc}
\left(E_{s}-E_{g}\right) \mathcal{I}_{4 \times 4} & 0 \\
0 & \left(E_{s}-E_{g}-\Delta\right) \mathcal{I}_{2 \times 2}
\end{array}\right)
\end{aligned}
$$

Onde as $\mathcal{I}_{2 \times 2}$ e $\mathcal{I}_{4 \times 4}$ são, respectivamente as matrizes identidade $2 \times 2$ e $4 \times 4$. O termo que acopla esses dois blocos é dado por (usando a notação $k_{ \pm}=k_{x} \pm i k_{y}$ ):

$$
W_{c v}=\left(\begin{array}{cccccc}
-\frac{1}{\sqrt{2}} P k_{+} & \sqrt{\frac{2}{3}} P k_{z} & \frac{1}{\sqrt{6}} P k_{-} & 0 & -\frac{1}{\sqrt{3}} P k_{z} & -\frac{1}{\sqrt{3}} P k_{-} \\
0 & -\frac{1}{\sqrt{6}} P k_{+} & \sqrt{\frac{2}{3}} P k_{z} & \frac{1}{\sqrt{2}} P k_{-} & -\frac{1}{\sqrt{3}} P k_{+} & \frac{1}{\sqrt{3}} P k_{z}
\end{array}\right)
$$

Por questão de hermiticidade do hamiltoniano, temos $W_{v c}=W_{c v}^{\dagger}$. Diagonalizando a matriz 2.17, é obtida a estrutura de bandas do material em questão através de seus autovalores $\epsilon_{n, \vec{k}}$. A partir das expressões analíticas das bandas podem-se obter as massas efetivas das respectivas bandas:

$$
\frac{1}{m_{i j}^{*}}=\frac{1}{\hbar^{2}} \frac{\partial \epsilon_{n, \vec{k}}}{\partial k_{i} \partial k_{j}}
$$


Comparando com resultados experimentais para as massas efetivas, pode ser extraído o valor dos elementos de matriz $P$ e $\Delta$. O modelo apresentado aqui, entretanto, não fornece os valores corretos para as massas efetivas, apresentando inclusive os sinais errados para as massas das bandas de valência. Para fazer ajustes experimentais e determinar o valor dos parâmetros $P$ e $\Delta$ é necessário usar modelos mais precisos com a inclusão da interação com bandas remotas. Para descrever a banda de condução, que é o que nos interessa no presente trabalho, o modelo é satisfatório[53].

O método $\vec{k} \cdot \vec{p}$ vale apenas para materiais em bulk. Em heteroestruturas há uma quebra da periodicidade da rede e uma generalização desse método, denominada Aproximação da Função Envelope, deve ser aplicada para tratar esses casos, o que será feito na próxima seção. O fato que pode ser antecipado é que, para esses casos, um hamiltoneano muito parecido com 2.17 é obtido, e os parâmetros dos materiais importantes na determinação dos elementos de matriz são os mesmos do caso bulk.

\subsection{EFA - Aproximação da Função Envelope}

Os cálculos feitos nas seções anteriores assumem que o material em questão é um cristal perfeito (e, portanto, infinito) e absolutamente periódico. Essas são excelentes hipóteses, caso estejamos lidando com material em bulk com o alto nível de pureza e ausência de defeitos que se pode conseguir com a tecnologia atual em semicondutores. Entretanto, a fabricação de dispositivos envolve a criação de interfaces entre diferentes materiais e a introdução de impurezas. Também a caracterização e medida de efeitos nesses dispositivos envolve aplicação de campos eletromagnéticos. Dessa forma, quebra-se a periodicidade desses sistemas, o que impede o uso do teorema de Bloch Eq. (2.4). Com o fim de tratar esse tipo de sistema, foi desenvolvida a Aproximação da Função Envelope (EFA - Envelope Function Approximation). As hipótese básicas para desenvolver essa aproximação são

- assume-se que as funções de onda na região do material $i$, possuem a forma:

$$
\Psi_{n, \vec{k}}^{i}=\sum_{n} F_{n}^{i}(\vec{x}) u_{n, \mathbf{0}}^{i}(\vec{x})
$$

onde as funções $u_{n, \mathbf{0}}^{i}$ são idênticas ao caso bulk e $F_{n}^{i}(\vec{x})$ são denominadas funções 
envelope, a serem determinadas,

- os campos externos variam em uma escala de distância muito maior que o parâmetro de rede do material, podendo ser considerados aproximadamente constantes dentro de cada cela unitária,

- as funções $u_{n, \mathbf{0}}^{i}(\vec{x})$ no ponto $\Gamma$ são idênticas para todos os materiais presentes na heteroestrutura.

Vamos então supor que à Eq.(2.3) seja adicionado um potencial não periódico $\phi(\vec{r})$ que varie lentamente e assumir o seguinte ansatz para a função de onda:

$$
|\Psi\rangle=\sum_{n} F_{n}(\vec{x})|n\rangle
$$

onde, dessa vez, os coeficientes de expanção $F_{n}$ não são mais constantes, mas operadores dados por funções do operador posição $\vec{x}$. Assumimos que esses coeficientes, a que denominaremos funções envelope, variam também lentamente como o potencial $\phi(\vec{x})$. Vamos substituir a Eq.(2.20) na equação de Schrödinger:

$$
[\mathcal{H}+\phi(\vec{x})]|\Psi\rangle=E|\Psi\rangle
$$

Sendo $\mathcal{H}=\frac{p^{2}}{2 m}+V(\vec{x})$ o hamiltoniano cristalino original ${ }^{13}$. Substituindo a Eq.(2.20):

$$
[\mathcal{H}+\phi(\vec{x})] \sum_{n} F_{n}(\vec{x})|n\rangle=\sum_{n} F_{n}(\vec{x})|n\rangle
$$

Fazendo o produto pela esquerda com $\langle m| F_{m}(\vec{x})$, temos:

$$
\sum_{n}\left\langle m\left|F_{m}(\vec{x})[\mathcal{H}+\phi(\vec{x})] F_{n}(\vec{x})\right| n\right\rangle=\sum_{n}\left\langle m\left|F_{m}(\vec{x}) F_{n}(\vec{x})\right| n\right\rangle
$$

Usando agora o resultado obtido no apêndice A, na Eq.(A.7), os seguintes elementos de matriz podem ser facilmente obtidos ${ }^{14}$, lembrando que as funções $u_{m, \mathbf{0}}(\vec{x})=\langle\vec{x} \mid m\rangle$ são

\footnotetext{
${ }^{13}$ Note novamente que a interação spin-órbita já está sendo considerada em primeira ordem através da escolha da base e das energias no ponto $\Gamma$.

${ }^{14}$ Realizamos o cálculo explicitamente no apêndice A, seção A.1.
} 
ortogonais:

$$
\begin{aligned}
\left\langle m\left|F_{m}(\vec{x}) F_{n}(\vec{x})\right| n\right\rangle & =\frac{\delta_{n m}}{\Omega} \int F_{m}(\vec{x}) F_{n}(\vec{x}) d^{3} x \\
\left\langle m\left|F_{m}(\vec{x}) \phi(\vec{x}) F_{n}(\vec{x})\right| n\right\rangle & =\frac{\delta_{n m}}{\Omega} \int F_{m}(\vec{x}) \phi(\vec{x}) F_{n}(\vec{x}) d^{3} x
\end{aligned}
$$

O elemento de matriz restante pode ser calculado notando-se que:

$$
\left[\mathcal{H}, F_{n}(\vec{x})\right]=\left[\frac{p^{2}}{2 m}, F_{n}(\vec{x})\right]=-\frac{\hbar^{2}}{2 m} \nabla^{2} F_{n}(\vec{x})-i \frac{\hbar}{m} \nabla F_{n}(\vec{x}) \cdot \vec{p}
$$

e portanto:

$$
\left\langle m\left|F_{m} \mathcal{H} F_{n}\right| n\right\rangle=\left\langle m\left|F_{m} F_{n} \mathcal{H}\right| n\right\rangle-\frac{\hbar^{2}}{2 m}\left\langle m\left|F_{m} \nabla^{2} F_{n}(\vec{x})\right| n\right\rangle-i \frac{\hbar}{m}\left\langle m\left|F_{m} \nabla F_{n}(\vec{x}) \cdot \vec{p}\right| n\right\rangle
$$

Uma nova aplicação do resultado da Eq.(A.7) e o uso do fato de que $|n\rangle$ é auto-estado de $\mathcal{H}$ fornece $^{15}$ :

$$
\left\langle m\left|F_{m} \mathcal{H} F_{n}\right| n\right\rangle=\frac{1}{\Omega} \int F_{m}(\vec{x})\left[\left(-\frac{\hbar^{2}}{2 m} \nabla^{2} F_{n}(\vec{x})+E_{n}^{0} F_{n}(\vec{x})\right) \delta_{m n}-\frac{i \hbar}{m} \nabla F_{n}(\vec{x}) \cdot \vec{p}_{m n}\right] d^{3} x
$$

onde os $\vec{p}_{m n}=\langle m|\vec{p}| n\rangle$ são os mesmos elementos de matriz que aparecem no caso bulk. Coletando todos os termos:

$$
\frac{1}{\Omega} \sum_{n} \int F_{m}(\vec{x})\left\{\left[-\frac{\hbar^{2}}{2 m} \nabla^{2} F_{n}(\vec{x})+\left(E_{n}^{0}-E+\phi(\vec{x})\right) F_{n}(\vec{x})\right] \delta_{m n}-\frac{i \hbar}{m} \nabla F_{n}(\vec{x}) \cdot \vec{p}_{m n}\right\}=0
$$

Dessa equação se obtém, postulando-se que os $F_{n}(\vec{x})$ sejam funções linearmente independentes:

$$
\left[-\frac{\hbar^{2}}{2 m} \nabla^{2} F_{m}(\vec{x})+\phi(\vec{x}) F_{m}(\vec{x})\right]-\frac{i \hbar}{m} \sum_{n} \vec{p}_{m n} \cdot \nabla F_{n}(\vec{x})=\left(E-E_{m}^{0}\right) F_{m}(\vec{x})
$$

Essa equação é muito similar à Eq.(2.11) se fizermos a correspondência $\vec{k} \rightarrow \frac{1}{i} \nabla$ e adicionarmos o termo relativo ao potencial $\phi(\vec{x})$ na diagonal.

\footnotetext{
${ }^{15}$ Cálculo explicitamente realizado na seção A.1 do apêndice A
} 


\subsection{Matriz de Kane em Heteroestruturas}

No caso de heteroestruturas, o potencial $\phi$ que deve aparecer nas equações acima está associado às descontinuidades nas bandas de valência e de condução. Dessa forma, esse potencial deve ter um perfil diferente em cada uma das bandas, associado aos diferentes offsets. Além disso, esse potencial estrutural deve variar apenas na direção de crescimento do cristal (que escolheremos na direção do eixo cartesiano z). Com isso, podemos fazer o seguinte ansatz para as funções envelope:

$$
F_{n}(\vec{x})=\frac{1}{\sqrt{A}} e^{i \vec{k}_{\|} \cdot \vec{x}_{\|}} f_{n}(z)
$$

onde $\vec{x}_{\| 1}$ denota a posição no plano xoy e $\vec{k}_{\| 1}=k_{x} \hat{x}+k_{y} \hat{y}$. Ficamos, finalmente, com uma equação muito similar ao caso bulk:

$$
\left(\begin{array}{cc}
H_{c} & W_{c v} \\
W_{c v} & H_{v}
\end{array}\right)\left(\begin{array}{c}
f_{c} \\
f_{v}
\end{array}\right)=E\left(\begin{array}{c}
f_{c} \\
f_{v}
\end{array}\right)
$$

onde, dessa vez, fazendo a associação:

$$
\hat{k}_{z}=\frac{\hbar}{i} \frac{d}{d z}
$$

temos:

$$
\begin{aligned}
& H_{c}=\left[\frac{\hbar^{2} \hat{k}_{z}^{2}}{2 m}+\frac{\hbar^{2} k_{\|}^{2}}{2 m}+V_{c}(z)\right] \mathcal{I}_{2 \times 2} \\
& H_{v}=\left(\begin{array}{cc}
\left(\frac{\hbar^{2} \hat{k}_{z}^{2}}{2 m}+\frac{\hbar^{2} k_{\|}^{2}}{2 m}+V_{v}(z)-E_{g}\right) \mathcal{I}_{4 \times 4} & 0 \\
0 & \left(\frac{\hbar^{2} \hat{k}_{z}^{2}}{2 m}+\frac{\hbar^{2} k_{\|}^{2}}{2 m}+V_{\Delta}(z)-E_{g}-\Delta\right) \mathcal{I}_{2 \times 2}
\end{array}\right)
\end{aligned}
$$

e o bloco $W_{c v}$ tem a mesma forma da Eq.(2.18) trocando $k_{z}$ por $\hat{k}_{z}$ e associando a parte do vetor $\vec{k}$ perpendicular ao eixo z com o vetor $\vec{k}_{\|}$definido acima. $f_{C}$ e $f_{V}$ são vetores coluna de 2 e 6 componentes respectivamente contendo as funções envelope referentes às bandas de condução e valência. Os potenciais $V_{c}(z), V_{v}(z)$ e $V_{\Delta}(z)$ são, respectivamente, os potenciais devido aos offsets estruturais das bandas de condução, bandas de buracos 
leves e pesados ${ }^{16}$ e bandas split-off.

\subsection{Aproximação de Hartree}

As equações do capítulo acima foram escritas assumindo que os elétrons no material são independentes, ou seja, que a função de onda de muitas partículas que descreve o sistema pode ser decomposta em um produto de funções de onda de uma partícula. Em casos em que a interação elétron-elétron é suficientemente importante para que efeitos de troca e correlação sejam observados essa suposição não é mais válida. Em casos em que apenas nos interessamos pelos primeiros efeitos da interação entre os elétrons podemos ainda fazer essa aproximação. A equação de Schrödinger original de muitas partículas ${ }^{17}$ é:

$$
\begin{aligned}
H \Psi\left(\vec{x}_{1}, \vec{x}_{2}, \ldots\right) & =\sum_{i}\left[\frac{p_{i}^{2}}{2 m}+u\left(\vec{x}_{i}\right)+\sum_{j} V\left(\vec{x}_{i}, \vec{x}_{j}\right)\right] \Psi\left(\vec{x}_{1}, \vec{x}_{2}, \ldots\right) \\
& =E \Psi\left(\vec{x}_{1}, \vec{x}_{2}, \ldots\right)
\end{aligned}
$$

onde os índices $i$ e $j$ variam de 1 até o número de elétrons, N, $u(\vec{x})$ é o potencial de interação com os íons da rede cristalina e $V\left(\vec{x}_{1}, \vec{x}_{2}\right)$ é o potencial de interação entre dois elétrons. Para determinar as energias desse sistema, faremos uma aproximação de elétrons independentes, ou seja, escreveremos a função de onda de muitas partículas como um produto de funções de uma única partícula. Neste ponto estamos ignorando efeitos de troca e correlação ${ }^{18}$. Essa hipótese pode ser escrita na forma:

$$
\Psi\left(\vec{x}_{1}, \vec{x}_{2}, \ldots\right)=\psi_{1}\left(\vec{x}_{1}\right) \psi_{2}\left(\vec{x}_{2}\right) \ldots \psi_{N}\left(\vec{x}_{N}\right)
$$

\footnotetext{
${ }^{16}$ Estamos assumindo que os offsets são iguais para buracos leves e pesados

${ }^{17}$ Note que aqui já foi aplicada a aproximação de Born-Oppenheimer.

${ }^{18}$ Estamos ignorando explicitamente o princípio de Pauli pois a função escolhida não é antissimétrica por troca de duas partículas.
} 
Usando essa função de onda, vamos construir o seguinte funcional:

$$
\begin{aligned}
\mathcal{E}=\langle\Psi|H| \Psi\rangle-E\langle\Psi \mid \Psi\rangle & =\sum_{i} \int \psi_{i}^{*}\left(\vec{x}_{i}\right)\left(\frac{p_{i}^{2}}{2 m}+u\left(\vec{x}_{i}\right)\right) \psi_{i}\left(\vec{x}_{i}\right) d^{3} x_{i}+ \\
& +\sum_{i j} \iint \psi_{i}^{*}\left(\vec{x}_{i}\right) \psi_{j}^{*}\left(\vec{x}_{j}\right) V\left(\vec{x}_{i}, \vec{x}_{j}\right) \psi_{i}\left(\vec{x}_{i}\right) \psi_{j}\left(\vec{x}_{j}\right) d^{3} x_{i} d^{3} x_{j}- \\
& -\sum_{i} E_{i} \int \psi_{i}^{*}\left(\vec{x}_{i}\right) \psi_{i}\left(\vec{x}_{i}\right) d^{3} x_{i}
\end{aligned}
$$

Aplicando o método variacional no funcional acima temos uma nova equação de Schrödinger:

$$
\frac{\delta \mathcal{E}}{\delta \psi_{k}^{*}(\vec{x})}=\left[\frac{p^{2}}{2 m}+u(\vec{x})+V_{\text {Hartree }}(\vec{x})\right] \psi_{k}(\vec{x})-E_{k} \psi_{k}(\vec{x})=0
$$

onde o potencial $V_{\text {Hartree }}(\vec{x})$, denominado potencial de Hartree, é dado por:

$$
V_{\text {Hartree }}(\vec{x})=\sum_{i} \int\left|\psi_{i}\left(\vec{x}^{\prime}\right)\right|^{2} V\left(\vec{x}, \vec{x}^{\prime}\right) d^{3} x^{\prime}
$$

No caso da interação de Coulomb, esse potencial será escrito como:

$$
V_{\text {Hartree }}(\vec{x})=\frac{e^{2}}{\kappa} \sum_{i} \int \frac{\left|\psi_{i}\left(\vec{x}^{\prime}\right)\right|^{2}}{\left|\vec{x}-\vec{x}^{\prime}\right|} d^{3} x^{\prime}
$$

Onde $\kappa$ é a constante dielétrica do material. Outra maneira de representar esse potencial é usar o fato de que ele é solução da equação de Poisson:

$$
\nabla^{2} V_{\text {Hartree }}(\vec{x})=-\frac{4 \pi e}{k} \rho(\vec{x})
$$

onde $\rho=-e \sum_{i}\left|\psi_{i}\right|^{2}$ é a densidade de carga eletrônica. Dessa forma, uma outra maneira de interpretar a aproximação de Hartree consiste em considerar que cada elétron interage com a densidade de carga eletrônica total por meio de um potencial elétrostático. No caso em que o gás de elétrons tem uma temperatura definida a densidade de carga deve ser calculada segundo:

$$
\rho(\vec{x})=-e \sum_{i} \frac{\left|\psi_{i}(\vec{x})\right|^{2}}{1+e^{\beta\left(\mu-E_{i}\right)}}
$$

Para heteroestruturas, vamos assumir que o potencial de Hartree pode ser separado em duas contribuições. A primeira varia na escala do parâmetro de rede e provém da variação nessa escala da densidade de cargas. Essa contribuição assumiremos estar somada ao 
potencial periódico da rede cujos efeitos já foram incluídos nos coeficientes $P, \Delta$ e $E_{g}$. A segunda contribuição varia em uma escala de distâncias muito maior, associada à escala das funções envelope. Esta contribuição será somada aos potenciais das bandas, transformando a Eq.(2.26) em:

$$
\begin{aligned}
& H_{c}=\left[\frac{\hbar^{2} \hat{k}_{z}^{2}}{2 m}+\frac{\hbar^{2} k_{\|}^{2}}{2 m}+V_{c}(z)+V(z)\right] \mathcal{I}_{2 \times 2} \\
& H_{v}=\left(\begin{array}{cc}
\left(\frac{\hbar^{2} \hat{k}_{z}^{2}}{2 m}+\frac{\hbar^{2} k_{\|}^{2}}{2 m}+V_{v}(z)+V(z)-E_{g}\right) \mathcal{I}_{4 \times 4} & 0 \\
0 & \left(\frac{\hbar^{2} \hat{k}_{z}^{2}}{2 m}+\frac{\hbar^{2} k_{\|}^{2}}{2 m}+V_{\Delta}(z)+V(z)-E_{g}-\Delta\right) \mathcal{I}_{2 \times 2}
\end{array}\right)
\end{aligned}
$$

A expressão correta para o potencial $V(z)$ será dada no capítulo 5 . 


\section{Acoplamento Spin-Órbita Inter Sub-banda}

\subsection{Introdução}

Neste capítulo aplicaremos o procedimento de "folding down" para isolar o setor do hamiltoniano da Eq. (2.25) correspondente à banda de condução[54]. Este procedimento é o mesmo usado na referência [10] para obter a aproximação não-relativística da equação de Dirac. Depois identificamos no hamiltoneano a parte correspondente à interação spinórbita na banda de condução e a parte correspondente ao movimento orbital e projetando tal hamiltoniano nas duas soluções de mais baixa energia da parte orbital encontramos uma matriz $4 \times 4$ que contém a interação spin-órbita de Rashba e um termo inter-subbanda que não se anula mesmo em estruturas simétricas. Obtemos também os autovetores e as autoenergias do sistema em estruturas simétricas e assimétricas.

\subsection{Folding Down}

O hamiltoniano apresentado na Eq.(2.25) pode ser transformado em um hamiltoniano 2x2 que descreve apenas a banda de condução por meio do procedimento denominado folding down. A equação citada pode ser escrita da seguinte forma:

$$
\begin{aligned}
& H_{c} f_{c}+W_{c v} f_{v}=E f_{c} \\
& H_{v} f_{v}+W_{v c} f_{c}=E f_{v}
\end{aligned}
$$


Podemos isolar $f_{v}$ em 3.2 e substituir em 3.1 eliminando assim essa componente:

$$
\begin{gathered}
f_{v}=\left(E-H_{v}\right)^{-1} W_{v c} f_{c} \\
{\left[H_{c}+W_{c v}\left(E-H_{v}\right)^{-1} W_{v c}\right] f_{c}=E f_{v}}
\end{gathered}
$$

A Eq.(3.3) é uma espécie de equação de Schrödinger para a banda de condução onde aparece um hamiltoniano efetivo $H_{e f}=H_{c}+W_{c v}\left(E-H_{v}\right)^{-1} W_{v c}$. No entanto essa equação não é um problema de autovalores comum pois o operador $H_{e f}$ depende da energia. Para tratar esse problema vamos considerar que as energias envolvidas são todas bem menores que o gap e que a energia de split-off ${ }^{1}$ e expandir o hamiltoniano efetivo $H_{\text {ef }}$ nesse limite. $\mathrm{O}$ operador $\left(H_{v}-E\right)^{-1}$ é dado por:

$$
\left(\begin{array}{cc}
\frac{1}{\eta_{1}} & 0 \\
0 & \frac{1}{\eta_{2}}
\end{array}\right)
$$

onde:

$$
\begin{aligned}
& \eta_{1}=E-\frac{\hbar^{2} \hat{k}_{z}^{2}}{2 m}-\frac{\hbar^{2} k_{\|}^{2}}{2 m}-V_{v}(z)-V(z)+E_{g} \\
& \eta_{2}=E-\frac{\hbar^{2} \hat{k}_{z}^{2}}{2 m}-\frac{\hbar^{2} k_{\|}^{2}}{2 m}-V_{\Delta}(z)-V(z)+E_{g}+\Delta
\end{aligned}
$$

Os potenciais $V_{i}(z)$ definem os perfis das bandas ao longo da estrutura. No caso de um poço quântico com uma barreira, eles são definidos por $^{2,3}$ :

$$
\begin{aligned}
V_{c}(z) & =\delta_{c} h(z)+\delta_{c}^{b} h_{b}(z) \\
V_{v}(z) & =-\delta_{v} h(z)-\delta_{v}^{b} h_{b}(z) \\
V_{\Delta}(z) & =-\delta_{\Delta} h(z)-\delta_{\Delta}^{b} h_{b}(z)
\end{aligned}
$$

As constantes $\delta_{j}^{i}$ são os off-sets entre as bandas dos diferentes materiais e estão definidas na figura 6(b). Voltaremos a essa discussão na seção 3.5.

\footnotetext{
${ }^{1} \mathrm{O}$ que equivale a manter o vetor $\vec{k}$ perto do ponto $\Gamma$

${ }^{2}$ Foi tomado como zero de energia o fundo da banda de condução do material de menor gap.

${ }^{3}$ Note que um poço quântico duplo pode ser tratado como um poço simples com uma barreira quadrada no centro.
} 


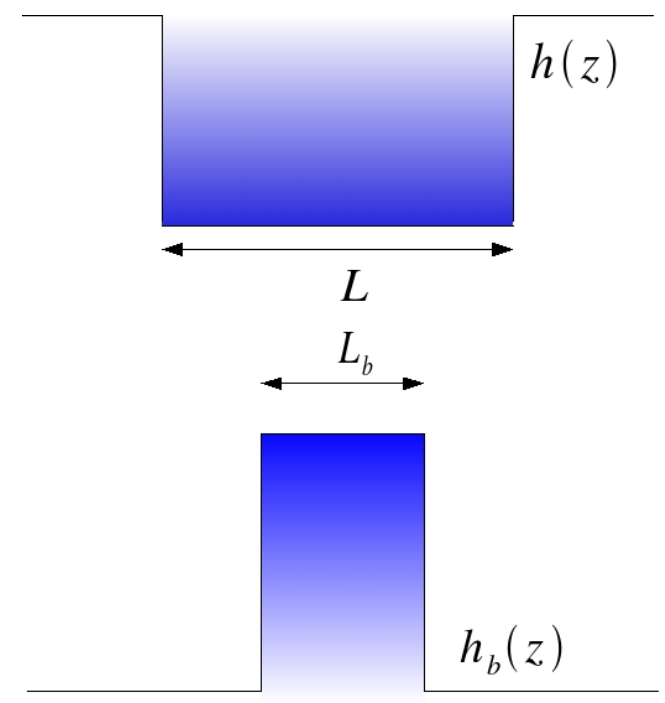

(a) Perfis $h(z)$ e $h_{b}(z)$. Os perfis são funções degrau adimensionais, com altura 1 e largura igual à largura do poço e da barreira respectivamente.

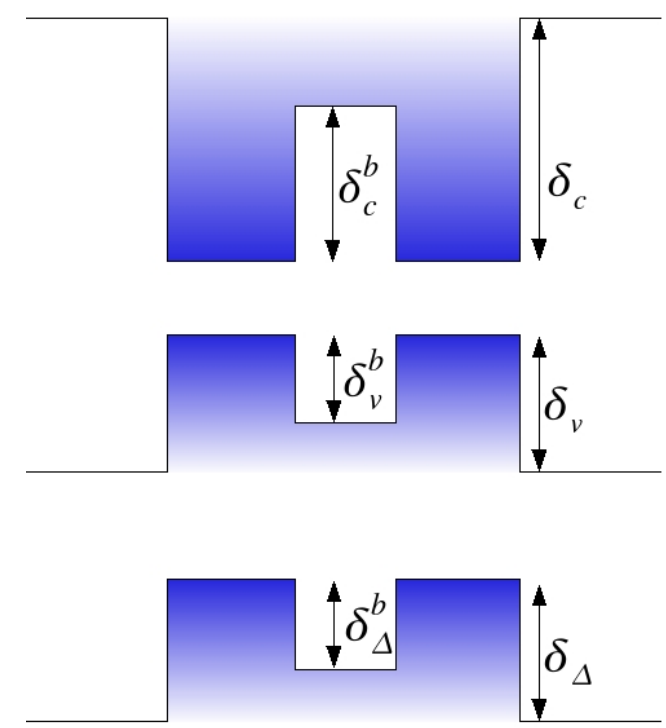

(b) Definição das energias $\delta_{i}^{j}$ correspondentes aos off-sets das diferentes bandas nos materiais que compõe o poço e as barreiras.

Figura 6: Perfis estruturais e off-sets das bandas. Na equação (3.4), os perfis e os off-sets são usados para definir os potenciais efetivos nas diferentes bandas.

Fazendo o produto $M=W_{c v}\left(H_{v}-E\right)^{-1} W_{v c}$ obtemos $^{4,5}$ :

$$
\begin{gathered}
M=\left\{\frac{P^{2} k_{\|}^{2}}{3}\left(\frac{2}{\eta_{1}}+\frac{1}{\eta_{2}}\right)+\frac{1}{3} k_{z}\left(\frac{2}{\eta_{1}}+\frac{1}{\eta_{2}}\right) k_{z}\right\} \mathcal{I}_{2 \times 2} \\
+\frac{P^{2}}{3} \frac{d}{d z}\left[\frac{1}{\eta_{1}}-\frac{1}{\eta_{2}}\right] \hat{z} \cdot\left(\vec{\sigma} \times \vec{k}_{\| 1}\right)
\end{gathered}
$$

sendo $\sigma_{x, y \text { ou } z}$ as matrizes de Pauli. Como dito antes, vamos expandir essa expressão para $E_{g}$ e $\Delta$ muito maiores que as outras escalas de energia. Para tal notemos que:

$$
\begin{aligned}
\frac{1}{\eta_{1}} & =\frac{1}{E-\frac{\hbar^{2} \hat{k}_{z}^{2}}{2 m}-\frac{\hbar^{2} k_{\|}^{2}}{2 m}-V_{v}(z)-V(z)+E_{g}} \\
\frac{1}{\eta_{1}} & =\frac{1}{E_{g}}\left[1-\frac{E-\frac{\hbar^{2} \hat{k}_{z}^{2}}{2 m}-\frac{\hbar^{2} k_{\|}^{2}}{2 m}-V_{v}(z)-V(z)}{E_{g}}+\ldots\right]
\end{aligned}
$$

\footnotetext{
${ }^{4}$ Aqui foi usado o fato de que $k_{ \pm}$são números e comutam com todas as outras variáveis e que $k_{+} k_{-}=k_{\|}^{2}$. Note também que o operador $k_{z}$ não comuta com $\eta_{1}$ e $\eta_{2}$

${ }^{5} \mathrm{O}$ símbolo $\mathcal{I}_{2 \times 2}$, como antes, representa a matriz identidade $2 \times 2$
} 
De maneira similar:

$$
\frac{1}{\eta_{2}}=\frac{1}{E_{g}+\Delta}\left[1-\frac{E-\frac{\hbar^{2} \hat{k}_{z}^{2}}{2 m}-\frac{\hbar^{2} k_{\|}^{2}}{2 m}-V_{\Delta}(z)-V(z)}{E_{g}+\Delta}+\ldots\right]
$$

Vamos manter em cada termo da Eq.(3.5) a ordem mais baixa não nula em $\frac{1}{E_{g}}$ e ${\frac{1}{E_{g}+\Delta}}^{6}$. Para o termo proporcional à identidade temos:

$$
\frac{2}{\eta_{1}}+\frac{1}{\eta_{2}}=\frac{2}{E_{g}}+\frac{1}{E_{g}+\Delta}
$$

Para o termo fora da diagonal temos ${ }^{7}$ :

$$
\frac{d}{d z}\left[\frac{1}{\eta_{1}}-\frac{1}{\eta_{2}}\right]=\left(\frac{V_{v}^{\prime}(z)}{E_{g}^{2}}-\frac{V_{\Delta}^{\prime}(z)}{\left(E_{g}+\Delta\right)^{2}}\right)+\left(\frac{1}{E_{g}^{2}}-\frac{1}{\left(E_{g}+\Delta\right)^{2}}\right) \frac{d V}{d z}
$$

\subsubsection{Massa Efetiva}

O hamiltoniano efetivo final possui uma parte diagonal dada por:

$$
\frac{\hbar^{2} \hat{k}_{z}^{2}}{2 m}+\frac{\hbar^{2} k_{\|}^{2}}{2 m}+V_{c}(z)+V(z)+\frac{P^{2}}{3}\left(k_{\|}^{2}+k_{z}^{2}\right)\left(\frac{2}{E_{g}}+\frac{1}{E_{g}+\Delta}\right)
$$

Todos os termos quadráticos no vetor de onda podem ser agrupados se definirmos uma massa efetiva:

$$
\frac{1}{m^{*}}=\frac{1}{m}+\frac{2 P^{2}}{3 \hbar^{2}}\left(\frac{2}{E_{g}}+\frac{1}{E_{g}+\Delta}\right)
$$

e escrevermos a parte diagonal do hamiltoniano como:

$$
\frac{\hbar^{2} \hat{k}_{z}^{2}}{2 m^{*}}+\frac{\hbar^{2} k_{\|}^{2}}{2 m^{*}}+V_{c}(z)+V(z)
$$

Esse procedimento ilustra o surgimento de uma massa efetiva em semicondutores. Massas efetivas surgem também no cálculo de propriedades de materiais em bulk. Entretanto, a definição de massa efetiva dada em (3.6) é inacurada pois ignora efeitos de acoplamento com bandas mais remotas que tem um efeito considerável sobre o seu valor. Para efeito de comparação, a razão $\frac{m_{*}}{m}$ para o GaAs é em torno de 0,067, enquanto a expressão acima fornece $\frac{m_{*}}{m}=0,053$. No presente trabalho será usado o valor experimental da massa efetiva

\footnotetext{
${ }^{6}$ Note que isso equivale a manter-se próximo ao ponto $\Gamma$.

${ }^{7} \mathrm{O}$ símbolo $f^{\prime}(z)$ aqui indica derivada da função $f(z)$ com relação à variável $z$.
} 
em todos os cálculos. Com essa definição, a expressão final para $H_{e f}$ é (omitindo a matriz identidade):

$$
H_{e f}=\frac{\hbar^{2} \hat{k}_{z}^{2}}{2 m^{*}}+\frac{\hbar^{2} k_{\Perp}^{2}}{2 m^{*}}+\Phi_{c}(z)+\eta(z) \hat{z} \cdot\left(\vec{\sigma} \times \vec{k}_{\| 1}\right)
$$

Onde definimos as funções:

$$
\eta(z)=\frac{P^{2}}{3}\left(\frac{\Phi_{v}^{\prime}(z)}{E_{g}^{2}}-\frac{\Phi_{\Delta}^{\prime}(z)}{\left(E_{g}+\Delta\right)^{2}}\right)
$$

e:

$$
\begin{aligned}
\Phi_{c}(z) & =V_{c}(z)+V(z) \\
\Phi_{v}(z) & =V_{v}(z)+V(z) \\
\Phi_{\Delta}(z) & =V_{\Delta}(z)+V(z)
\end{aligned}
$$

para simplificar a notação.

\subsection{Solução da parte orbital}

O hamiltoniano da Eq.(3.7) possui duas partes distintas: a parte proporcional à identidade, a que vamos nos referir como parte orbital, e o termo $\eta(z) \hat{z} \cdot\left(\vec{\sigma} \times \vec{k}_{\| 1}\right)$, que contém a interação spin-órbita.Podemos tratar esse problema separadamente: primeiro encontrar uma base de autoestados da parte orbital do hamiltoniano da Eq.(3.7) e depois encontrar uma representação matricial para esse mesmo hamiltoniano nessa base ${ }^{8}$. As autofunções orbitais devem satisfazer uma equação de Schrödinger:

$$
-\frac{\hbar^{2}}{2 m^{*}} \frac{d^{2} \psi_{n}(z)}{d z^{2}}+\Phi_{c}(z) \psi_{n}(z)=E_{n} \psi_{n}(z)=\left(\epsilon_{n, \vec{k}_{\|}}-\frac{\hbar^{2} k_{\Perp}^{2}}{2 m^{*}}\right) \psi_{n}(z)
$$

Essa equação fornece uma série de níveis discretos na direção de confinamento z que se soma ao espectro contínuo proveninente do movimento livre no plano xy. Assim se formam

\footnotetext{
${ }^{8}$ Note que esta é uma maneira aproximada de encarar o problema pois as condições de contorno do problema original são dependentes de spin. Cálculos semelhantes foram feitos nas referências [55, 56] comparando os resultados com e sem a inclusão de condições de contorno dependentes de spin. As diferenças são suficientemente pequenas para justificar a aproximação. Entretanto, o efeito dessas pequenas diferenças em cálculos que incluem o termo de troca e correlação não pode ser prevista e talvez as condições de contorno dependentes de spin afete propriedades de sistemas como os estudados nas referências [57, 58] onde efeitos coletivos como o ferromagnetismo de efeito Hall são importantes.
} 
uma seqüência de sub-bandas contínuas como mostra a Fig. $(7)^{9}$

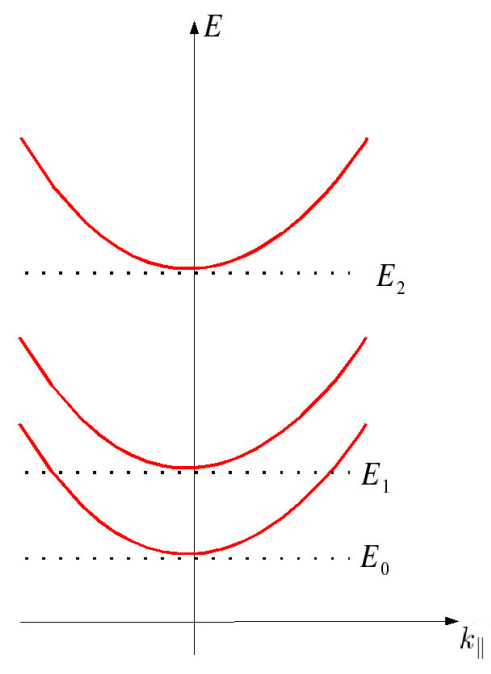

Figura 7: Autoenergias da parte orbital em função de $k_{\|}$mostrando as sub-bandas contínuas

\subsection{Sistemas com duas sub-bandas}

Com uma engenharia adequada do potencial estrutural pode-se conseguir os dois primeiros níveis de energia da parte orbital bem próximos entre si e distantes de outros eventuais níveis que possam existir. Nessa situaçao podemos ignorar o acoplamento entre os estados mais distantes e escrever a matriz que representa o hamiltoniano total na base $\{|0,+\rangle,|0,-\rangle,|1,+\rangle,|1,-\rangle\}$ obtendo um hamiltoneano $4 \mathrm{x} 4$. Para calcular os elementos de matriz, notemos que:

$$
\left\langle j, \sigma_{1}\left|\eta(z) \hat{z} \cdot\left(\vec{\sigma} \times \vec{k}_{\text {II }}\right)\right| l, \sigma_{2}\right\rangle=\langle j|\eta(z)| l\rangle\left\langle\sigma_{1}\left|\hat{z} \cdot\left(\vec{\sigma} \times \vec{k}_{\| 1}\right)\right| \sigma_{2}\right\rangle
$$

Se definirmos:

$$
\begin{aligned}
\alpha_{0} & =\langle 0|\eta(z)| 0\rangle \\
\alpha_{1} & =\langle 1|\eta(z)| 1\rangle \\
\eta & =\langle 0|\eta(z)| 1\rangle \\
k_{ \pm} & =k_{x} \pm i k_{y}
\end{aligned}
$$

\footnotetext{
${ }^{9}$ Cada sub-banda é duplamente degenerada devido ao spin
} 
e notarmos que:

$$
\begin{aligned}
& \left\langle \pm\left|\hat{z} \cdot\left(\vec{\sigma} \times \vec{k}_{11}\right)\right| \pm\right\rangle=0 \\
& \left\langle\mp\left|\hat{z} \cdot\left(\vec{\sigma} \times \vec{k}_{\| 1}\right)\right| \pm\right\rangle=\mp i k_{ \pm}
\end{aligned}
$$

A representação matricial de $H_{e f}$ fica:

$$
H_{e f}=\left(\begin{array}{cccc}
\epsilon_{0}+\frac{\hbar^{2} k_{\|}^{2}}{2 m^{*}} & i k_{-} \alpha_{0} & 0 & i k_{-} \eta \\
-i k_{+} \alpha_{0} & \epsilon_{0}+\frac{\hbar^{2} k_{\|}^{2}}{2 m^{*}} & -i k_{+} \eta & 0 \\
0 & i k_{-} \eta & \epsilon_{1}+\frac{\hbar^{2} k_{1}^{2}}{2 m^{*}} & i k_{-} \alpha_{1} \\
-i k_{+} \eta & 0 & -i k_{+} \alpha_{1} & \epsilon_{1}+\frac{\hbar^{2} k_{\|}^{2}}{2 m^{*}}
\end{array}\right)
$$

Caso o sistema tenha simetria de inversão com relação ao eixo z, as autofunções $\langle z \mid 0\rangle$ e $\langle z \mid 1\rangle$ da parte orbital têm paridades opostas. A de menor autovalor deve ser par, e nesse caso indicaremos a função por $\psi_{e}(z)=\langle z \mid e\rangle^{10}$ e o primeiro excitado deve ser ímpar, sendo indicado por $\psi_{o}(z)=\langle z \mid o\rangle$. Neste caso todos os potenciais devem ser pares e suas derivadas ímpares. Dessa forma o integrando na expressão que define $\alpha_{0}$ e $\alpha_{1}$ é ímpar e portanto ambas as constantes são nulas. Nesse caso é possível tornar o hamiltoniano bloco-diagonal se escolhermos a base na ordem $|e+\rangle,|o-\rangle,|o+\rangle,|e-\rangle$. Com esse reordenamento da base temos:

$$
H_{e f}=\left(\begin{array}{cc|cc}
\epsilon_{0}+\frac{\hbar^{2} k_{\|}^{2}}{2 m^{*}} & i k_{-} \eta & \multicolumn{2}{|c}{0} \\
-i k_{+} \eta & \epsilon_{1}+\frac{\hbar^{2} k_{\|}^{2}}{2 m^{*}} & & \\
\hline 0 & \epsilon_{1}+\frac{\hbar^{2} k_{\|}^{2}}{2 m^{*}} & i k_{-} \eta \\
& -i k_{+} \eta & \epsilon_{0}+\frac{\hbar^{2} k_{n}^{2}}{2 m^{*}}
\end{array}\right)
$$

A constante $\eta$ determina portanto a intensidade de um acoplamento spin-órbita entre estados pertencentes a sub-bandas diferentes. O objetivo final desse trabalho é determinar autoconsistentemente o valor da constante $\eta$ para várias estruturas para fornecer uma parametrização adequada para o hamiltoneano 3.11.

\footnotetext{
${ }^{10}$ Notação induzida pelas palavras inglesas even e odd
} 


\subsection{Potenciais}

Os potenciais $\Phi_{v}(z)$ e $\Phi_{\Delta}(z)$ que aparecem na Eq.(3.8) possuem duas partes: a originária das descontinuidades na estrutura de banda ao longo do sistema e o potencial de Hartree, como descrito na seção (2.6). Neste trabalho estaremos interessados em poços quânticos simples e duplos. Para uma descrição unificada destes dois tipos de sistemas, é interessante notar que o potencial de um poço duplo pode ser escrito simplesmente como o de um poço simples mais uma barreira quadrada. Quando a altura da barreira quadrada é nula, temos um poço simples.

Assim, em termos dos off-sets (ver figura 6(b)) das diferentes bandas, os potenciais podem ser escritos como:

$$
\begin{aligned}
\Phi_{c}(z) & =V(z)+\delta_{c} h(z)+\delta_{c}^{b} h_{b}(z) \\
\Phi_{v}(z) & =V(z)-\delta_{v} h(z)-\delta_{v}^{b} h_{b}(z) \\
\Phi_{\Delta}(z) & =V(z)-\delta_{\Delta} h(z)-\delta_{\Delta}^{b} h_{b}(z)
\end{aligned}
$$

Sendo $h(z)$ e $h_{b}(z)$ perfis adimensionais que descrevem o poço e a barreira respectivamente, como indicado na figural 6(a). Substituindo esses potenciais na Eq.(3.8) ficamos com:

$\eta(z)=\frac{P^{2}}{3}\left[\left(\frac{1}{E_{g}^{2}}-\frac{1}{\left(E_{g}+\Delta\right)^{2}}\right) V^{\prime}(z)-\left(\frac{\delta_{v}}{E_{g}^{2}}-\frac{\delta_{\Delta}}{\left(E_{g}+\Delta\right)^{2}}\right) h^{\prime}(z)-\left(\frac{\delta_{v}^{b}}{E_{g}^{2}}-\frac{\delta_{\Delta}^{b}}{\left(E_{g}+\Delta\right)^{2}}\right) h_{b}^{\prime}(z)\right]$

Portanto a constante de acoplamento $\eta$ pode ser escrita como consistindo de 3 contribuições provientes respectivamente do potencial de Hartree, do perfil que descreve o poço e do perfil que descreve a barreira ${ }^{11}$ :

$$
\begin{aligned}
\eta_{\text {poço }} & =-\frac{P^{2}}{3}\left(\frac{\delta_{v}}{E_{g}^{2}}-\frac{\delta_{\Delta}}{\left(E_{g}+\Delta\right)^{2}}\right)\left\langle e\left|h^{\prime}(z)\right| o\right\rangle \\
\eta_{\text {barreira }} & =-\frac{P^{2}}{3}\left(\frac{\delta_{v}^{b}}{E_{g}^{2}}-\frac{\delta_{\Delta}^{b}}{\left(E_{g}+\Delta\right)^{2}}\right)\left\langle e\left|h_{b}^{\prime}(z)\right| o\right\rangle \\
\eta_{\text {Hartree }} & =\frac{P^{2}}{3}\left(\frac{1}{E_{g}^{2}}-\frac{1}{\left(E_{g}+\Delta\right)^{2}}\right)\left\langle e\left|V^{\prime}(z)\right| o\right\rangle
\end{aligned}
$$

As fórmulas para $\eta_{\text {poço }}$ e $\eta_{\text {barreira }}$ podem ser ainda simplificadas se notarmos que as derivadas

\footnotetext{
${ }^{11}$ As formulas da equação (3.13) valem apenas para o caso de um poço quântico com uma barreira central (poço quântico duplo).
} 
dos perfis $h(z)$ e $h_{b}(z)$ são conjuntos de funções delta. Na situação simétrica ficamos com:

$$
\left\langle e\left|h^{\prime}(z)\right| o\right\rangle=\int \psi_{e}(z)[-\delta(z+L / 2)+\delta(z-L / 2)] \psi_{o}(z) d z=2 \psi_{e}\left(\frac{L}{2}\right) \psi_{o}\left(\frac{L}{2}\right)
$$

Similarmente:

$$
\left\langle e\left|h_{b}^{\prime}(z)\right| o\right\rangle=\int \psi_{e}(z)\left[\delta\left(z+L_{b} / 2\right)-\delta\left(z-L_{b} / 2\right)\right] \psi_{o}(z) d z=-2 \psi_{e}\left(\frac{L_{b}}{2}\right) \psi_{o}\left(\frac{L_{b}}{2}\right)
$$

Sobre o potencial de Hartree $V_{H}(z)$ acima é preciso dizer que, no tratamento numérico realizado, foi usada uma definição ligeiramente diferente da implementada na seção 2.6. Incluimos neste potencial, além do efeito de interação entre os elétrons, os potenciais elétricos devido a regiões dopadas com doadores de carga e potenciais externos aplicados através de eletrodos.

\subsection{Novas relações de dispersão}

\subsubsection{Autofunções e Autoenergias: estruturas simétricas}

$\mathrm{Na}$ ausência de assimetria, os dois blocos do hamiltoniano dado acima em (3.11) são trivialmente diagonalizados possuindo os mesmos autovalores. O conjunto de autovalores e autovetores é dado na tabela 2 , usando as definições: $\epsilon_{ \pm}=\frac{\epsilon_{1} \pm \epsilon_{0}}{2}, \theta=\arctan \left(\frac{\eta k_{\| 1}}{\epsilon_{-}}\right) \mathrm{e}$ $\phi=\arctan \left(\frac{k_{x}}{k_{y}}\right)$.

Tabela 2: Autoenergias e autofunções para o caso simétrico

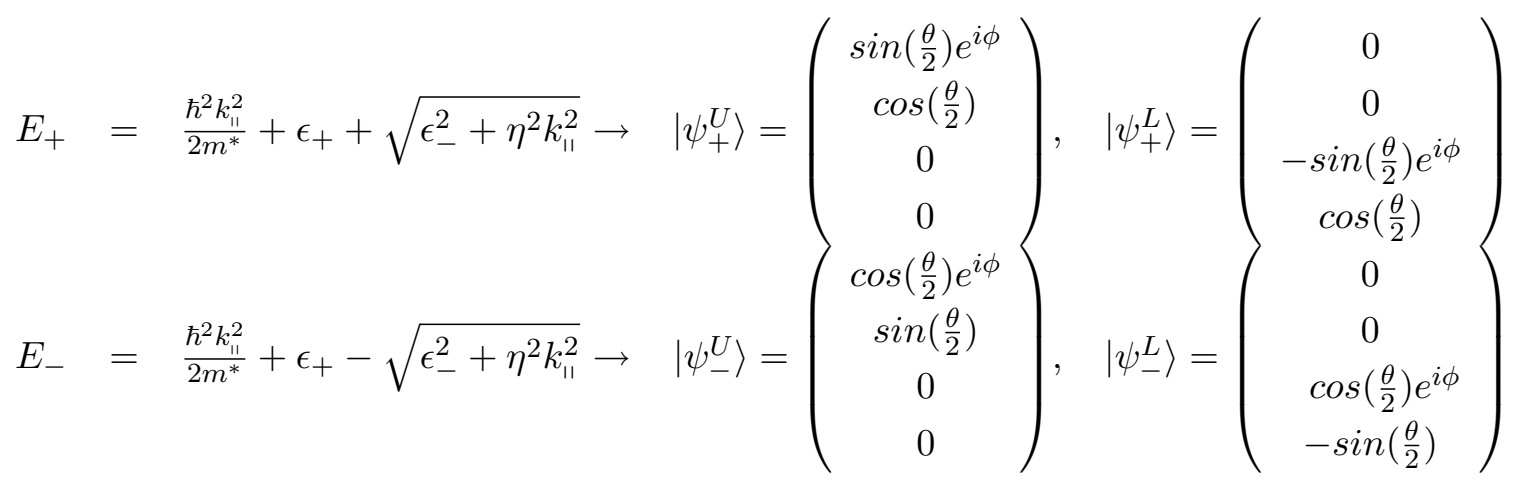




\subsubsection{Pequenos valores de $\eta$ : renormalização da massa}

Para pequenos valores de $\eta^{12}$ a expressão para as energias pode ser expandida em segunda ordem nessa grandeza resultando na expressão:

$$
E_{p m}=\left(\epsilon_{+} \pm \epsilon_{-}\right)+\frac{\hbar^{2} k_{\Perp 1}^{2}}{2 m_{ \pm}^{*}}
$$

onde as novas massa efetivas $m_{ \pm}^{*}$ são dadas por:

$$
m_{ \pm}^{*}=m^{*}\left(1 \mp \frac{2 \epsilon_{s o}}{\epsilon_{-}}\right)
$$

e o parâmetro $\epsilon_{s o}$ tem unidade de energia e é dado por:

$$
\epsilon_{s o}=\frac{1}{2} m^{*}\left(\frac{\eta}{\hbar}\right)^{2}
$$

\subsubsection{Relação de dispersão na presença de assimetria}

O hamiltoneano da Eq.(3.10) pode ser simplificado escolhendo-se uma base adequada para o spin. Tomando da Eq.(3.7), podemos usar a propriedade cíclica do produto triplo ${ }^{13}$ e escrever o hamiltoneano efetivo como:

$$
H_{e f}=\frac{\hbar^{2} \hat{k}_{z}^{2}}{2 m^{*}}+\frac{\hbar^{2} k_{\|}^{2}}{2 m^{*}}+\Phi_{c}(z)+\eta(z) \vec{\sigma} \cdot\left(\vec{k}_{\|} \times \hat{z}\right)=\frac{\hbar^{2}}{2 m^{*}}\left(\hat{k}_{z}^{2}+k_{\|}^{2}\right)+\Phi_{c}(z)+\vec{\sigma} \cdot \overrightarrow{\mathcal{B}}\left(z, \vec{k}_{\|}\right)
$$

Com a equação nesta forma, o acoplamento spin órbita pode ser interpretado como uma espécie de efeito Zeeman devido a um campo magnético no plano, $\overrightarrow{\mathcal{B}}\left(z, \vec{k}_{11}\right)$, dependente de $z$ e $\vec{k}$. Esse termo "Zeeman" pode ser escrito como:

$$
\begin{aligned}
\eta(z) \vec{\sigma} \cdot\left(\vec{k}_{\|} \times \hat{z}\right) & =\eta(z) \vec{\sigma} \cdot\left(-k_{x} \hat{y}+k_{y} \hat{x}\right) \\
& =k_{\Perp} \eta(z) \vec{\sigma} \cdot \hat{r}=k_{\Perp} \eta(z) \sigma_{r}
\end{aligned}
$$

Onde o versor $\hat{r}$ é dado por:

$$
\hat{r}=-k_{x} / k_{\|} \hat{y}+k_{y} / k_{\|} \hat{x}
$$

\footnotetext{
${ }^{12}$ pequenos valores da razão $\frac{\eta^{2} k_{\|}^{2}}{\epsilon_{-}}$, ou seja, para a região próxima do ponto $\vec{k}=0$.

${ }^{13}$ Uma vez que $\vec{\sigma}, \vec{k}$ e $\hat{z}$ comutam entre si.
} 
O operador $\sigma_{r}$ é dado por:

$$
\sigma_{r}=\vec{\sigma} \cdot \hat{r}=\left(\begin{array}{cc}
0 & \frac{k_{y}+i k_{x}}{k_{\|}} \\
\frac{k_{y}-i k_{x}}{k_{\|}} & 0
\end{array}\right) \equiv\left(\begin{array}{cc}
0 & e^{i \theta} \\
e^{-i \theta} & 0
\end{array}\right)
$$

Onde definimos $e^{i \theta}=\frac{k_{y}+i k_{x}}{k_{\|}}$. Os autovalores desse operador são \pm 1 e seus autovetores são dados por:

$$
| \pm\rangle_{r}=\frac{1}{\sqrt{2}}\left(\begin{array}{c}
0 \\
\pm e^{i \theta}
\end{array}\right)
$$

Usando $| \pm\rangle_{r}$ como como base para o spin, a matriz que representa o hamiltoneano 3.17 é:

$$
H_{e f}=\left(\begin{array}{cc}
\frac{\hbar^{2}}{2 m^{*}}\left(\hat{k}_{z}^{2}+k_{\|}^{2}\right)+\Phi_{c}(z)+k_{\|} \eta(z) & 0 \\
0 & \frac{\hbar^{2}}{2 m^{*}}\left(\hat{k}_{z}^{2}+k_{\|}^{2}\right)+\Phi_{c}(z)-k_{\|} \eta(z)
\end{array}\right)
$$

Podemos projetar essa matriz nas duas primeiras subbandas como feito anteriormente e obter:

$$
H_{e f}=\left(\begin{array}{c|cc}
\epsilon_{0}+\frac{\hbar^{2} k_{\|}^{2}}{2 m^{*}}+k_{\|} \alpha_{0} & k_{\|} \eta & 0 \\
k_{\|} \eta & \epsilon_{1}+\frac{\hbar^{2} k_{\| 1}^{2}}{2 m^{*}}+k_{\|} \alpha_{1} & \\
\hline 0 & \epsilon_{0}+\frac{\hbar^{2} k_{\|}^{2}}{2 m^{*}}-k_{\|} \alpha_{0} & -k_{\| 1} \eta \\
-k_{\| 1} \eta & \epsilon_{1}+\frac{\hbar^{2} k_{\|}^{2}}{2 m^{*}}-k_{\|} \alpha_{1}
\end{array}\right)
$$

Da matriz da Eq.(3.21) pode ser extraída a relação de dispersão:

$$
E_{\sigma, \lambda}(\vec{k})=\frac{\hbar^{2} k_{\|}^{2}}{2 m}+\epsilon_{+}+\sigma \alpha_{+} k_{\|}+\lambda \sqrt{\eta^{2} k_{\|}^{2}+\left(\epsilon_{-}-\sigma \alpha_{-} k_{\|}\right)^{2}}
$$

Os números quânticos $\sigma$ e $\lambda$ podem ser \pm 1 e representam, respectivamente, o graus de liberdade de spin na direção ${ }^{14} \hat{r}$ e uma espécie de pseudo-spin. As constantes de acoplamento de Rashba dão origem às constantes $\alpha_{ \pm}=\frac{\alpha_{0} \pm \alpha_{1}}{2}$. Escolhendo $\alpha_{i}=0$ essa relação de dispersão recupera as expressões do caso simétrico (equações da tabela 2), como esperado. Os autovetores serão dados por:

$$
|\sigma, \lambda\rangle=| \pm\rangle_{r} \otimes|\sigma, \lambda\rangle_{2}
$$

\footnotetext{
${ }^{14}$ Note que a direção $\hat{r}$ é dependente do vetor de onda $k_{\|}$
} 
onde:

$$
|\sigma, \lambda\rangle_{2}=\frac{1}{\sqrt{2\left(1+\lambda \sin \left(\phi_{\sigma}\right)\right)}}\left(\begin{array}{c}
\cos \phi_{\sigma} \\
\sin \phi_{\sigma}+\lambda
\end{array}\right)
$$

e:

$$
\cos \phi_{\sigma}=\frac{\sigma k_{\|} \eta}{\sqrt{k_{\|}^{2} \eta^{2}+\left(\epsilon_{-}+\sigma \alpha_{-} k_{\|}\right)}}
$$

Substituindo as expressões 3.25 e 3.19, na expressão 3.23 temos:

$$
|\sigma, \lambda\rangle=\frac{1}{2} \frac{1}{\sqrt{1+\lambda \sin \left(\phi_{\sigma}\right)}}\left(\begin{array}{c}
\cos \phi_{\sigma} \\
\sin \phi_{\sigma}+\lambda \\
\sigma \cos \phi_{\sigma} e^{i \theta} \\
\sigma\left(\sin \phi_{\sigma}+\lambda\right) e^{i \theta}
\end{array}\right)
$$




\section{Escolha dos Materiais e Estruturas}

O objetivo deste trabalho é a determinação autoconsistente da constante de acoplamento spin-órbita inter-subbanda e da constante de Rashba para diversas estruturas e diferentes materiais. Neste capítulo são apresentados os critérios para a escolha dos sistemas e materiais para os quais o cálculo foi realizado.

\subsection{Sistemas a Serem Simulados}

O modelo apresentado até agora vale para gases de elétrons quasi-bidimensionais (2DEG - 2 Dimensional Electron Gases) confinados em heteroestruturas. O sistema mais simples que se pode imaginar que apresenta essas caracteristicas é o poço quântico apresentado na Fig. 8. O material $\mathrm{BC}$ é um composto binário semicondutor e, nas regiões em azul, o elemento A entra como uma impureza substitucional com a mesma valência do elemento $\mathrm{B}$, mantendo o material neutro. O exemplo mais tradicional e mais bem caracterizado experimentalmente de sistema desse tipo são as heteroestruturas do tipo $\mathrm{Al}_{\mathrm{x}} \mathrm{Ga}_{1-\mathrm{x}} \mathrm{As} / \mathrm{GaAs} / \mathrm{Al}_{\mathrm{x}} \mathrm{Ga}_{1-\mathrm{x}} \mathrm{As}$. Esse poço tem um número finito ${ }^{1}$ de estados ligados determinado pelos valores de $V_{0}$ e $L$ que podem ser classificados com um número quântico $n$, em ordem crescente de energia. Conforme $n$ aumenta, os estados ficam cada vez mais espaçados. Escolhendo adequadamente os valores de $V_{0}$ e $L$ pode-se conseguir uma situação compatível com o modelo apresentado: um poço com apenas dois níveis de energia confinados no melhor dos casos, ou dois níveis confinados próximos e um terceiro bem mais

\footnotetext{
${ }^{1}$ A solução exata de um poço simples para o caso de elétrons não interagentes pode ser encontrada na referência [59], no capítulo X
} 


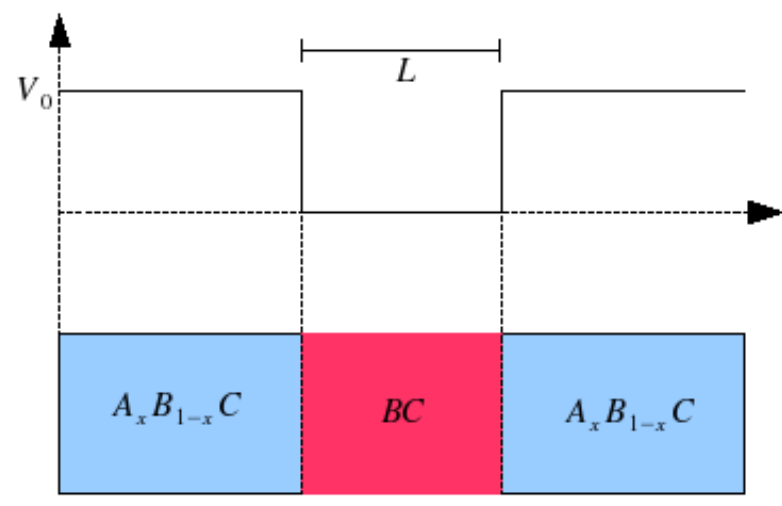

Figura 8: Um poço quântico simples e suas variáveis estruturais importantes: o tamanho do poço $L$ e a profundidade do potencial $V_{o}$

distante.

Pode-se aproximar bastante em energia os dos primeiros níveis e afastá-los do terceiro incluindo uma barreira central e construindo um poço duplo (DQW - Double Quantum Well). Numa visão de "tight-binding" pode-se imaginar que os dois primeiros níveis de energia desse sistema serão uma combinação simétrica e uma anti-simétrica das autofunções de cada poço separadamente ${ }^{2}$. A separação entre esses níveis de energia será proporcional ao overlap (sobreposição) entre essas funções de onda localizadas em cada poço e será tanto menor quanto mais alta ou mais larga for a barreira. Um eventual terceiro nível surgiria, nessa visão, de uma combinações de estados com energia mais alta e em geral estará muito mais distante do primeiro par de níveis em comparação a distância entre estes. Dessa forma, por uma questão de coerência com o modelo apresentado no capítulo anterior e pela possibilidade de minimizar a diferença de energia entre os dois níveis (maximizando assim o efeito da interação spin-órbita intersubbanda sobre a massa efetiva), os poços quânticos duplos parecem ser os candidatos mais promissores para a medida desse efeito.

Os materiais considerados nesse trabalho são semicondutores intrínsecos e, portanto, isolantes a temperatura 0K. Possuem uma banda de valência totalmente preenchida e uma banda de condução vazia. Para popular a banda de condução com elétrons nos sistemas aqui considerados introduz-se regiões dopadas com doadores de elétrons simetricamente adjacentes ao poço quântico ${ }^{3}$. A Fig.(9) ilustra um poço quantico duplo com regiões de

\footnotetext{
${ }^{2}$ Similar ao caso de orbitais ligantes e anti-ligantes discutido na página 29

${ }^{3}$ Uma substituição do elemento do grupo III por um elemento do grupo IV no caso de semicondutores
} 
doadores simetricamente posicionadas nas barreiras externas. Vamos supor ao longo desse trabalho que o poço quântico é suficientemente profundo para que todos os doadores sejam ionizados e a camada seja completamente depletada formando duas regiões com densidade de carga positiva ao redor do poço.

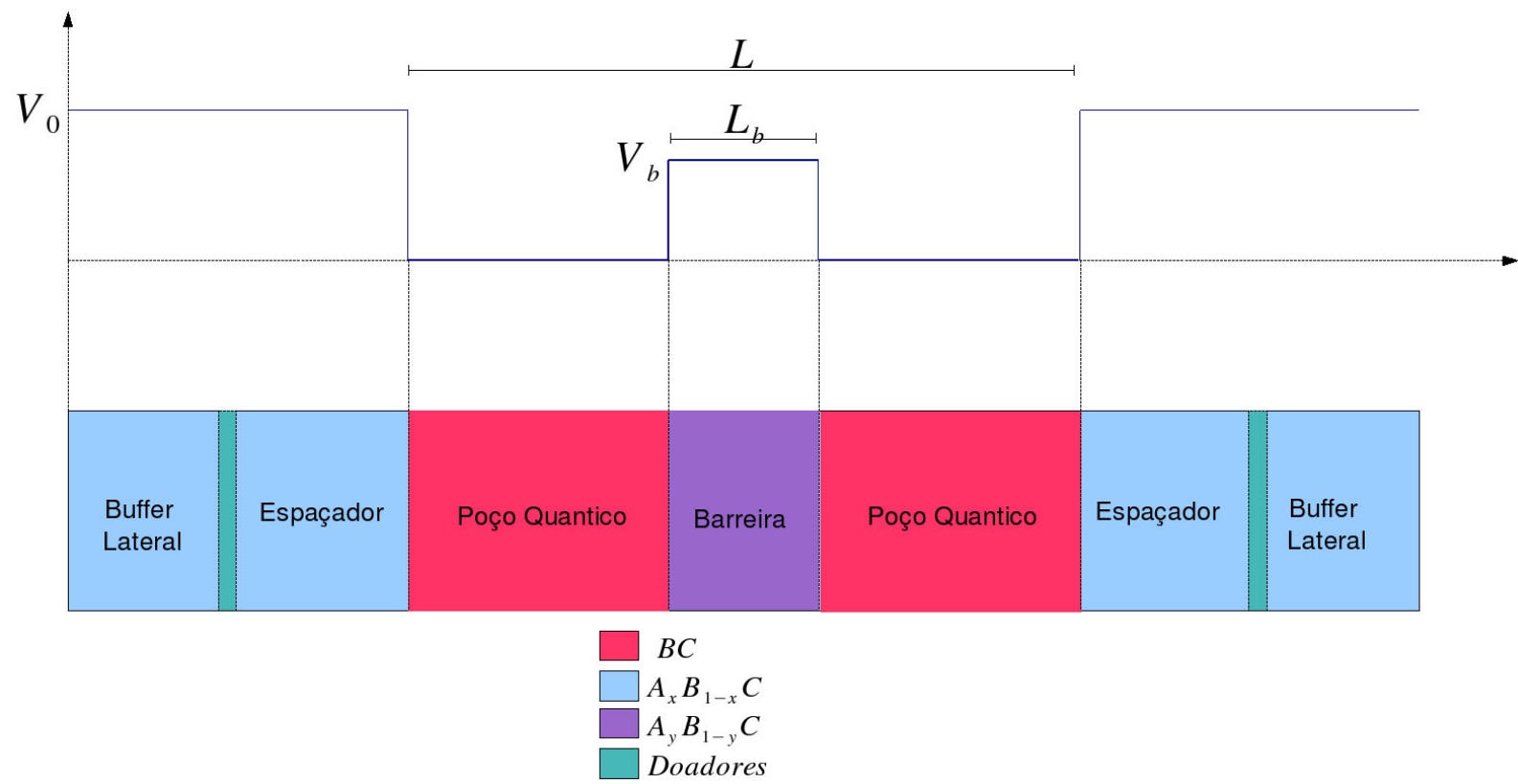

Figura 9: Um exemplo de poço quântico duplo e variáveis estruturais relevantes. Além das já presentes no poço simples se incluem o a altura $V_{b}$ e largura $L_{b}$ da barreira. A altura $V_{b}$ pode ser maior ou menor do que a altura da barreira lateral $V_{0}$

\subsection{Materiais}

A expressão de $\eta$ envolve, como visto, parâmetros da estrutura de bandas bulk dos materiais em questão como o gap, a energia de split-off e os off-sets das diferentes bandas. Percebe-se na Eq.(3.13) duas condições importantes dependentes unicamente do material usado e não da heteroestrutura para aumentar o valor de $\eta$ : gap pequeno e energia de split-off grande. Essas mesmas condições valem para o acoplamento Rashba [17, 32, 34] e se aplicam aqui devido à semelhança na origem dos dois acoplamentos. Dessa forma espera-se que o material que apresente o maior $\eta$ sejam as ligas que contém InSb, que do grupo III-V. Por exemplo substituição de Ga por Si no GaAs. 
possui o menor gap e o maior $\Delta_{S O}$ entre os as ligas binárias semicondutoras do grupo $I I I-V^{4}$. Por essa razão simulamos heteroestruturas de $\mathrm{Al}_{\mathrm{x}} \operatorname{In}_{1-\mathrm{x}} \mathrm{Sb} / \mathrm{InSb} / \mathrm{Al}_{\mathrm{x}} \mathrm{In}_{1-\mathrm{x}} \mathrm{Sb}$. Outro material escolhido para simulação para efeito de comparação foram heteroestruturas de $\mathrm{Al}_{\mathrm{x}} \mathrm{Ga}_{1-\mathrm{x}} \mathrm{As} / \mathrm{GaAs} / \mathrm{Al}_{\mathrm{x}} \mathrm{Ga}_{1-\mathrm{x}} \mathrm{As}$, por ser o material mais bem caracterizado experimentalmente. Trabalhos experimentais recentes $[60,61]$ mostram que o efeito do acoplamento

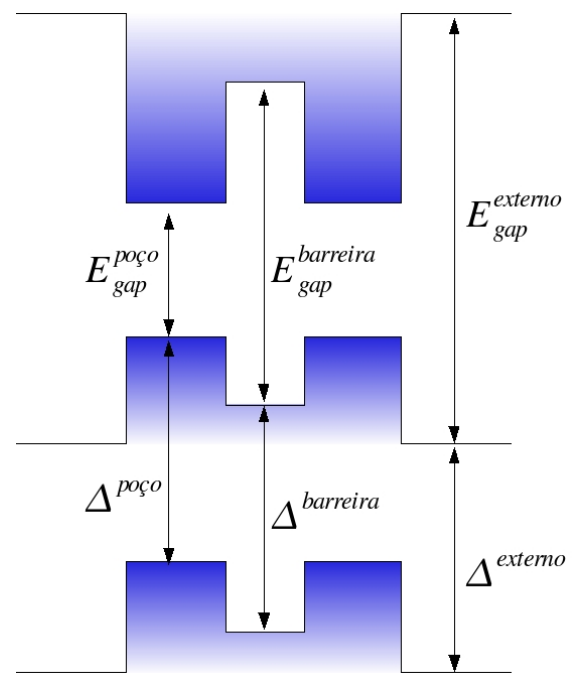

Figura 10: Estrutura de banda de um poço duplo mostrando os valores do gap e da energia split-off ao longo da estrutura.

Rashba é dominante sobre o acoplamento Dresselhaus em poços quânticos de GaAs e InAs. Não vamos portanto considerar a segunda forma de acoplamento neste trabalho por questão de simplicidade.

\subsubsection{Parâmetros de Estrutura de Banda}

Todos os valores de gap's, energias de split-off, off-sets de bandas, massas efetivas e outros parâmetros relacionados à estrutura de bandas dos materiais selecionados usados nas simulações, são os recomendados pela referência [53]. Para ligas ternárias foi usado o procedimento indicado na mesma referência: para o material $A_{x} B_{1-x} C$, o valor do gap e da energia split-off é dado pela interpolação:

$$
E\left[A_{x} B_{1-x} C\right]=x E[A C]+(1-x) E[B C]-x(1-x) C_{E}[A B C]
$$

\footnotetext{
${ }^{4}$ Ver referência [53] - o gap do InSb é de apenas $235 \mathrm{meV}$ e a energia de split-off é de $810 \mathrm{meV}$
} 
onde $C_{E}[A B C]$ é uma parâmetro que depende de cada material, denominado parâmetro de abaulamento de banda (bowing parameter). Na tabela 3 são apresentados os valores dos parâmetros necessários à simulação recomendados pela referência [53] para as ligas binárias citadas. Os parâmetros de abaulamento para as ligas ternárias são dados na tabela 4 . O

Tabela 3: Parâmetros de estrutura de bandas de ligas binárias III-V.

\begin{tabular}{lccc} 
Materiais: & & InSb & GaAs \\
\hline \hline Tipo de Gap: & & direto & direto \\
$E_{\text {gap }}^{\Gamma}$ & $(\mathrm{eV})$ & 0.235 & 1.519 \\
$\Delta$ & $(\mathrm{eV})$ & 0.810 & 0.341 \\
$E_{P}$ & $(\mathrm{eV})$ & 23.3 & 28.8 \\
$m^{*} / m_{0}$ & adim & 0.0135 & 0.067 \\
Materiais: & & AlSb & AlAs \\
\hline \hline Tipo de Gap: & & indireto & indireto \\
$E_{\text {gap }}^{\Gamma}$ & $(\mathrm{eV})$ & 1.696 & 3.099 \\
$\Delta$ & $(\mathrm{eV})$ & 0.676 & 0.28
\end{tabular}

parâmetro $E_{P}$ dado na tabela tem a forma como normalmente é informado na literatura o valor do elemento de matriz de Kane $P$. Esse parâmetro tem unidade de energia e é dado por:

$$
E_{P}=\frac{2 m}{\hbar^{2}} P^{2}
$$

Todos os materiais considerados nesse trabalho pertencem ao grupo III-V, possuem gap direto $^{5}$ e estrutura cristalina do tipo zincblende.

Tabela 4: Parâmetros de abaulamento e Valence Band Offset(VBO) para ligas ternárias

\begin{tabular}{lccc}
\multicolumn{2}{c}{ Materiais: } & $A l_{x} I n_{1-x} S b$ & $A l_{x} G a_{1-x} A s$ \\
\hline \hline$C_{\text {gap }}$ & $(\mathrm{eV})$ & 0.43 & $-0.127+1.310 x$ \\
$C_{\Delta}$ & $(\mathrm{eV})$ & 0.25 & 0 \\
VBO & adim & 0.19 & 0.34
\end{tabular}

Para determinar o potencial estrutural é preciso ainda saber como as posições relativas

\footnotetext{
${ }^{5}$ Alguns dos materiais da tabela 3 tem gap indireto. Eles foram inclusos na tabela porque são usados na determinação dos parâmetros de ligas ternárias de gap direto. Para esses materiais o gap fornecido na tabela corresponde sempre à diferença de energia entre as bordas da banda de valência e de condução no ponto $\Gamma$. Apenas são informados para esses materiais os parâmetros usados na interpolação.
} 
em energia dos extremos das bandas variam com a concentração do material A na liga $\mathrm{A}_{\mathrm{x}} \mathrm{B}_{1-\mathrm{x}} \mathrm{C}$. O parâmetro que determina essa grandeza é conhecido como Valence Band Offset(VBO) e é definido como a razão entre o deslocamento da banda de valência com relação ao caso $x=0$ e a variação do gap. Denotando a posição do topo da banda de valência em função de $x$ como $E_{v}(x)$, temos:

$$
\mathrm{VBO}=\frac{E_{v}(x)-E_{v}(0)}{E_{g}(x)-E_{g}(0)}
$$

É um fato experimental[53] que para muitas ligas o valor de VBO é uma constante que depende apenas dos elementos $A, B$ e $C$, e é independente da concentração $x$. O valor para os VBO's das ligas usadas nesse trabalho são dados na tabela 4 


\section{Cálculo Autoconsistente e Aspectos Computacionais}

A inclusão do potencial de Hartree no cálculo da estrutura eletrônica do poço quântico exige a implementação de um método iterativo conhecido como cálculo autoconsistente. Nesse capítulo introduzimos o conceito de autoconsistência e os métodos numéricos usados para a solução do problema.

\subsection{Formulação do Problema}

A parte que varia lentamente do potencial de Hartree $V(z)$ que aparece na equação $(3.9)^{1}$ ainda não foi definida precisamente. Vamos assumir que esse potencial é resultante de uma densidade de cargas associada às funções envelope da banda de condução ${ }^{2}$ :

$$
\begin{aligned}
\frac{d^{2} V}{d z^{2}} & =-\frac{4 \pi e^{2}}{\kappa}\left[\rho_{e}(z)+\rho_{d}(z)\right] \\
\rho_{e}(z) & =-2 e \sum_{n, \vec{k}_{\|}} \frac{\left|\Psi_{n, \vec{k}_{\|}}(\vec{x})\right|^{2}}{1+e^{\beta\left(\epsilon_{n, \vec{k}_{\|}}-\mu\right)}}
\end{aligned}
$$

Onde as funções envelope ${ }^{3}$ são dadas por $\Psi_{n, \vec{k}_{\|}}(\vec{x})=\frac{1}{\sqrt{A}} \psi_{n}(z) e^{i \vec{k}_{\|} \cdot \vec{x}}$, as energias dadas por $\epsilon_{n, \vec{k}_{\|}}=\frac{\hbar^{2} k_{\|}^{2}}{2 m^{*}}+E_{n}$ e a densidade de cargas $\rho_{d}(z)$ que descreve os doadores ionizados é uma função degrau, com um valor positivo $N_{d}$ na região dos doadores e nula fora dessa região. $N_{d}$ é o número de elétrons fornecidos pelos doadores por volume de região dopada.

\footnotetext{
${ }^{1}$ Implicito na definição de $\Phi_{c}(z)$

${ }^{2}$ Procurar referência [48], capítulo $\mathrm{V}$

${ }^{3} A$ é a área do gás de elétrons no plano $x y$.
} 
Simultaneamente a essas equações deve-se resolver a equação de Schrödinger para a função envelope $\psi_{n}(z)^{4}$ :

$$
-\frac{\hbar^{2}}{2 m^{*}} \frac{d^{2} \psi_{n}(z)}{d z^{2}}+\left[V_{c}(z)+V(z)\right] \psi_{n}(z)=E_{n} \psi_{n}(z)=\left(\epsilon_{n, \vec{k}_{\|}}-\frac{\hbar^{2} k_{\|}^{2}}{2 m^{*}}\right) \psi_{n}(z)
$$

A soma em $\vec{k}_{\|}$da equação (5.2) converte-se em uma integral introduzindo a densidade de pontos no espaço recíproco bidimensional, como de praxe ${ }^{5}$, e substituido a função envelope $\Psi_{n, \vec{k}_{\|}}$pela expressão dada acima:

$$
\rho_{e}(z)=-\frac{2 e A}{(2 \pi)^{2}} \sum_{n} \frac{1}{A}\left|\psi_{n}(z)\right|^{2} \iint \frac{1}{1+e^{\beta\left(\epsilon_{n, \vec{k}}-\mu\right)}} d^{2} k
$$

Uma vez que a energia depende apenas do módulo do vetor de onda, a integral angular é trivial:

$$
\rho_{e}(z)=-\frac{e}{\pi} \sum_{n}\left|\psi_{n}(z)\right|^{2} \int_{0}^{\infty} \frac{1}{1+\exp \left[\beta\left(\frac{\hbar^{2} k^{2}}{2 m^{*}}+E_{n}-\mu\right)\right]} k d k
$$

Essa integral resolve-se exatamente ${ }^{6}$ e seu resultado é:

$$
\rho_{e}(z)=-\frac{e}{\beta \hbar^{2} \pi} \sum_{n}\left|\psi_{n}(z)\right|^{2} \log \left[1+e^{\beta\left(\mu-E_{n}\right)}\right]
$$

\subsubsection{Condições de Contorno na Equação de Poisson}

A equação (5.1) demanda duas condições de contorno para ser resolvida. Para simular a aplicação de potenciais externos que possam quebrar a simetria de inversão da estrutura, vamos implementar condições de contorno de Dirichlet:

$$
\begin{aligned}
& V\left(+\frac{L}{2}\right)=V_{\text {front }} \\
& V\left(-\frac{L}{2}\right)=V_{\text {back }}
\end{aligned}
$$

\footnotetext{
${ }^{4}$ Equação (3.9)

${ }^{5}$ Ver referência [49]

${ }^{6}$ Através da transformação de variáveis $u=\frac{\hbar^{2} k^{2}}{2 m^{*}}$, e notando que $\int[1+\exp (\beta(u-X))]^{-1} d u=$ $\frac{1}{\beta} \log [1+\exp \beta X]$
} 
Amostras reais são muito maiores do que apenas a região de interesse em torno dos poços quânticos e dos doadores. Seria impraticável para o atual trabalho simular toda a estrutura dado o grau de precisão com que é necessário obter a função de onda na região de interesse. Dessa forma os potenciais $V_{\text {front }}$ e $V_{\text {back }}$ não correspondem aos potenciais aplicados nos eletrodos externos (gates). Não é possível prever exatamente no modelo aqui apresentado as quedas de potencial dentro das regiões que não são incluídas na simulação. Portanto esses potenciais apenas simulam de forma qualitativa a aplicação de um campo elétrico cuja função é quebrar a simetria de inversão.

\subsection{Potencial Químico e Densidade Eletrônica}

O potencial químico $\mu$ na equação (5.4) não está ainda determinado. É preciso aplicar alguma condição para determiná-lo. Dois modelos foram usados no presente trabalho:

- Modelo de reservatório

- Modelo da carga total constante

\subsubsection{Modelo de reservatório}

Na fabricação de estruturas é possível criar um reservatório de elétrons distante do poço que fixa o potencial químico eletrônico em toda a amostra. Assim é possível regular a densidade de elétrons dentro do poço através dos gates externos. Usaremos esse modelo para simular a aplicação de um potencial externo. O potencial químico será inicialmente calculado assumindo que o poço é neutro na situação simétrica e esse mesmo potencial químico será mantido constante durante a simulação variando-se o potencial dos eletrodos.

\subsubsection{Modelo da Carga total constante}

Caso não hajam esses reservatórios externos de elétrons e a camada de doadores tenha sido completamente depletada, passamos a um outro regime: a carga total dentro do poço quântico é constante e o potencial químico é determinado de tal modo a satisfazer essa 
quantidade de carga. Caso os potenciais $V_{\text {front }}$ e $V_{\text {back }}$ sejam iguais e não haja assimetria alguma, assumiremos que a carga total dentro do sistema é constante. Na simulações consideramos a carga total nula, ou seja, a situação em que todos os elétrons que ocupam os estados dentro do poço são provenientes da região dopada com doadores e não há nenhuma outra fonte de portadores. Dessa forma teremos:

$$
\begin{gathered}
\int\left(\rho_{e}+\rho_{d}\right) d^{3} x=0 \\
A \int \rho_{e}(z) d z+A \int \rho_{d}(z) d z=0
\end{gathered}
$$

A integral da densidade de cargas positiva dos doadores é facilmente realizada se notarmos que a densidade é constante e positiva nas duas regiões definidas pelos doadores de largura, digamos, $w_{d}$. Assim, substituindo a expressão (5.4), temos:

$$
-\frac{e}{\beta \hbar^{2} \pi} \sum_{n} \log \left[1+e^{\beta\left(\mu-E_{n}\right)}\right] \int\left|\psi_{n}(z)\right|^{2} d z+2 N_{d} w_{d}=0
$$

Assumindo que as funções $\psi_{n}(z)$ são normalizadas, ficamos finalmente com:

$$
\sum_{n} \log \left[1+e^{\beta\left(\mu-E_{n}\right)}\right]=2 N_{d} w_{d} \frac{\beta \hbar^{2} \pi}{e}
$$

Essa equação pode ser usada para determinar numericamente o valor de $\mu$.

\subsection{Cálculo Autoconsistente}

Todas as equações até agora estão acopladas de maneira inevitável. É preciso saber as funções envelope $\psi_{n}(z)$ para se calcular a densidade de carga e é preciso saber as densidades de carga para se determinar o potencial de Hartree e em última instância, as próprias funções envelope. Essa situação é ilustrada no diagrama da figura 11. Não é possível resolver essas equações simultaneamente em um método numérico único. É preciso implementar um método de aproximações sucessivas conhecido como Cálculo Autoconsistente. Em primeiro lugar é feita uma estimativa inicial para o potencial de Hartree $V(z)$. No nosso caso, escolhemos $V(z)=0$ inicialmente. Essa estimativa então é levada à equação de Schrödinger (5.3) e são determinadas as funções envelope e energias. Essas funções 


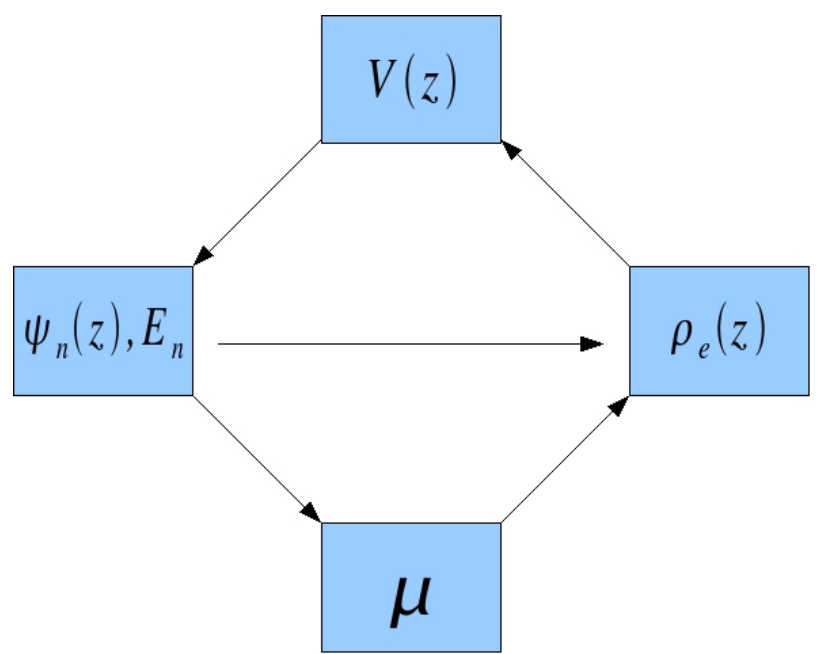

Figura 11: Diagrama mostrando a interdependência das variáveis. Uma variável na base de uma seta é necessária para determinar a variável na ponta da mesma seta. A solução para esse problema é um método de aproximações sucessivas conhecido como Cálculo Autoconsistente

e energias são usadas para se determinar o potencial químico e a densidade de cargas e, a partir da densidade de cargas, um novo potencial $V(z)$. O novo potencial é inserido na equação de Schrödinger e o procedimento é seguido sucessivamente até que se atinja a convergência, ou seja, até que as energias e funções de onda no próximo passo sejam muito próximas das energias e funções de onda do passo anterior. O critério usado para convergência na simulação feita é:

$$
\begin{aligned}
\int\left|\psi_{0}^{i}(z)-\psi_{0}^{i-1}(z)\right|^{2} d z & <10^{-10} \\
\mu^{i}-\mu^{i-1} & <10^{-10} \mathrm{meV}
\end{aligned}
$$

Isso garante que tanto as funções envelope como as energias estão convergidas com precisão suficiente.

\subsection{Métodos Numéricos}

Para resolver a equação de Schrödinger (5.3), a equação de Poisson (5.1) e a equação para o potencial químico (5.6) implementou-se diversos métodos numéricos. Nesta seção descreveremos cada um deles e suas peculiaridades. 


\subsubsection{Equação de Schrödinger - Método de Numerov}

O método de Numerov é um esquema para solução de equações diferenciais de segunda ordem do tipo:

$$
\frac{d^{2} \psi}{d z^{2}}=G(\psi, z)
$$

cujo erro é proporcional à sexta potência do passo de discretização. Discretizando o intervalo real por um passo constante h, o esquema para propagar a equação ao longo da discretização é dado por:

$$
\psi(z \pm h)=\frac{2[1+5 g(z)] \psi(z)-[1-g(z \mp h)] \psi(z-h)}{1-g(z \pm h)}
$$

Onde $g(z)=\frac{h^{2}}{12} G(\psi(z), z)$. No caso da equação de Schrödinger, a função $G(\psi(z), z)$ é dada por $[62]$ :

$$
G(\psi(z), z)=-\frac{2 m}{\hbar^{2}}[E-V(z)] \psi(z)
$$

e além de determinar $\psi(z)$, é necessário determinar as energias $E$ para as quais a função satizfaça as condições de contorno:

$$
\psi\left(-\frac{L}{2}\right)=\psi\left(+\frac{L}{2}\right)=0
$$

Além disso $\psi(z)$ e $\frac{d \psi}{d z}$ devem ser contínuas ao longo de todo o intervalo de integração. O procedimento para resolver essa equação pelo método de Numerov tem duas partes. Em primeiro lugar devemos selecionar qual autoenergia nos interessa. É sabido que a equação de Schrödinger unidimensional possui várias soluções satisfazendo (5.10)e que se ordenarmos as suas soluções em ordem crescente de energia, a n-ésima função $\psi(z)$ possui n-1 raízes reais (que chamaremos 'nós') no intervalo $-\frac{L}{2}<z<\frac{L}{2}$. Portanto é necessário inicialmente repartir o intervalo de energias de interesse em regiões que nos fornecem soluções com 0 nós, 1 nó, 2 nós,... assim sucessivamente. Posteriormente seleciona-se uma estimativa para a energia $E$ dentro do intervalo correspondente ao número de nós desejado e duas funções são criadas: a função $\psi_{d}(z)$ é obtida propagando-se a equação através do esquema 5.9 a partir de $z=-\frac{L}{2}$ para a direita, a função $\psi_{e}(z)$ é obtida propagando-se a equação a partir de $z=\frac{L}{2}$ para a esquerda. Ambas as funções são normalizadas para que em um certo 
ponto $^{7} z_{m}$ tenha-se $\psi_{e}\left(z_{m}\right)=\psi_{d}\left(z_{m}\right)=1$. Se a estimativa inicial para a energia estivesse correta, teríamos:

$$
\frac{d \psi_{e}}{d z}\left(z_{m}\right)=\frac{d \psi_{d}}{d z}\left(z_{m}\right)
$$

entretanto, como a estimativa inicial normalmente é grosseira, essa condição não é satisfeita. É possível mostrar que a diferença entre a energia correta e a estimativa dada é proporcional à diferença na derivada das duas funções no ponto $z_{m}$ :

$$
\Delta E \approx \frac{\hbar^{2}}{2 m}\left(\int_{-L / 2}^{L / 2} \psi(z)^{2} d z\right)^{-1}\left(\frac{d \psi_{e}}{d z}\left(z_{m}\right)-\frac{d \psi_{d}}{d z}\left(z_{m}\right)\right)
$$

Assim, somando a correção ${ }^{8}$ dada por $\Delta E$ à estimativa inicial para a energia temos uma estimativa melhorada. Pode-se repetir esse processo até que a energia e a função $\psi(z)$ tenham convergido para valores dentro de uma certa tolerância exigida, de maneira semelhante ao critério da equação (5.7). Observa-se que é preciso tomar cuidado para que a correção $\Delta E$ não leve a energia para fora do intervalo correspondente ao número de nós desejado. Para isso, normalmente escala-se a correção dada pela equação 5.11 por um fator da ordem de um décimo.

Discussões mais detalhadas e um pacote algébrico que implementa a solução da equação de Schrödinger usando o método de Numerov podem ser encontradas na referência [62].

\subsubsection{Equação de Poisson - Método de Numerov adaptado}

O método discutido na seção anterior pode ser adaptado para a solução da equação de Poisson (5.1) com condições de contorno de Dirichlet dadas por (5.5). A dedução do esquema de propagação da solução é dado na referência [63]. Dada uma equação do tipo:

$$
\begin{gathered}
\frac{d^{2} \phi}{d z^{2}}=-\rho(z) \\
\phi(-L / 2)=C_{1}, \phi(L / 2)=C_{2}
\end{gathered}
$$

\footnotetext{
${ }^{7}$ É desejável que se escolha $z_{m}$ coincidindo com um máximo de uma das duas funções. Isso torna o algoritmo mais estável.

${ }^{8} \mathrm{~A}$ integral $\int_{-L / 2}^{L / 2} \psi(z)^{2} d z$ pode ser estimada como $\int_{-L / 2}^{z_{m}} \psi_{d}(z)^{2} d z+\int_{z_{m}}^{L / 2} \psi_{e}(z)^{2} d z$
} 
Discretizando o intervalo em $N+1$ pontos igualmente espaçados por um passo h, e usando a notação $f_{i}=f\left(x_{i}\right)$, a solução é obtida através do esquema:

$$
\begin{aligned}
& U_{i}=\rho_{i}+10 \rho_{i-1}+\rho_{i-2} \\
& V_{i}=V_{i-1}+U_{i} \\
& Y_{i}=i U_{i} \\
& Z_{i}=Z_{i-1}+Y_{i} \\
& \phi_{2}=\frac{C_{2}}{N}+\left(1-\frac{1}{N}\right) C_{1}+h^{2} \sum_{j=3}^{N+1}(N+2-j) \rho_{j-1} \\
& \phi_{i}=(i-1) \phi_{2}-(i-2) C_{1}-\frac{h^{2}}{12}\left[(i+1) V_{i}-Z_{i}\right]
\end{aligned}
$$

O erro associado ao método continua sendo de sexta ordem no passo $h$ e o tempo de computação é linear com o número de pontos.

\subsubsection{Equação para o potencial químico}

A equação (5.6) pode ser escrita na forma:

$$
F\left(\mu, a_{1}, a_{2}, \ldots\right)=0
$$

onde $a_{i}$ são parâmetros conhecidos (energias, massa efetiva, constantes fundamentais, etc...). O potencial químico desejado é então a única raíz dessa função. Para encontrar essa raíz foi usado o código disponível na referência [64] para o algoritmo de Van Wijngaarden-Dekker-Brent de busca de raízes de equações não-lineares. O método converge de maneira garantida caso se conheça um intervalo que contém a raíz desejada e o faz no mínimo tão rapidamente quanto o algoritmo de bisecção, tendo melhor desempenho que o mesmo caso a função seja bem comportada. 


\section{$6 \quad$ Resultados}

A partir do modelo teórico apresentado nas seções anteriores, determinou-se as constantes de acoplamento inter-subbanda e de Rashba para diversas estruturas, a procura de valores experimentalmente mensuráveis. Simulamos poços quânticos simples (SQW - Single Quantum Well) e duplos (DQW - Double Quantum Well), e os materiais considerados foram GaAs e InSb e suas ligas com Al. Variou-se os parâmetros estruturais disponíveis na tentativa de encontrar valores ótimos para $\eta$ e os maiores valores possíveis para a razão $\frac{\epsilon_{S O}}{E_{o}-E_{e}}{ }^{1}$. Nas seções seguintes apresentam-se gráficos das constantes de acoplamento em função de parâmetros como largura e altura dos poços quânticos e barreiras, densidades de portadores, e potenciais elétricos externos. Escolhemos na apresentação dos gráficos unificar a descrição de SQW's e DQW's, uma vez que ambos os tipos de poços são descritos usando-se a concentração do elemento de liga² na barreira, que em essência determina a altura da mesma, como parâmetro.

\subsection{Poços simétricos simples e duplos}

\subsection{1 $\eta$ vs. tamanho do poço quântico}

A Fig.12 traz o resultado da variação de $\eta$ com o tamanho do poço para três poços duplos simétricos com barreiras centrais de diferentes tamanhos e um poço sem barreira central. Os parâmetros relevantes da simulação são dados na tabela 5. O gráfico nos mostra a tendência da constante de acoplamento em diminuir em módulo com o aumento da largura do poço. Na Fig. 13, podemos ver as diversas componentes de $\eta$ para os sistemas

\footnotetext{
${ }^{1} \mathrm{Na}$ tentativa de encontrar um efeito substancial de renormalização das massas das subbandas.

${ }^{2} \mathrm{Em}$ todos os casos se trata de alumínio.
} 


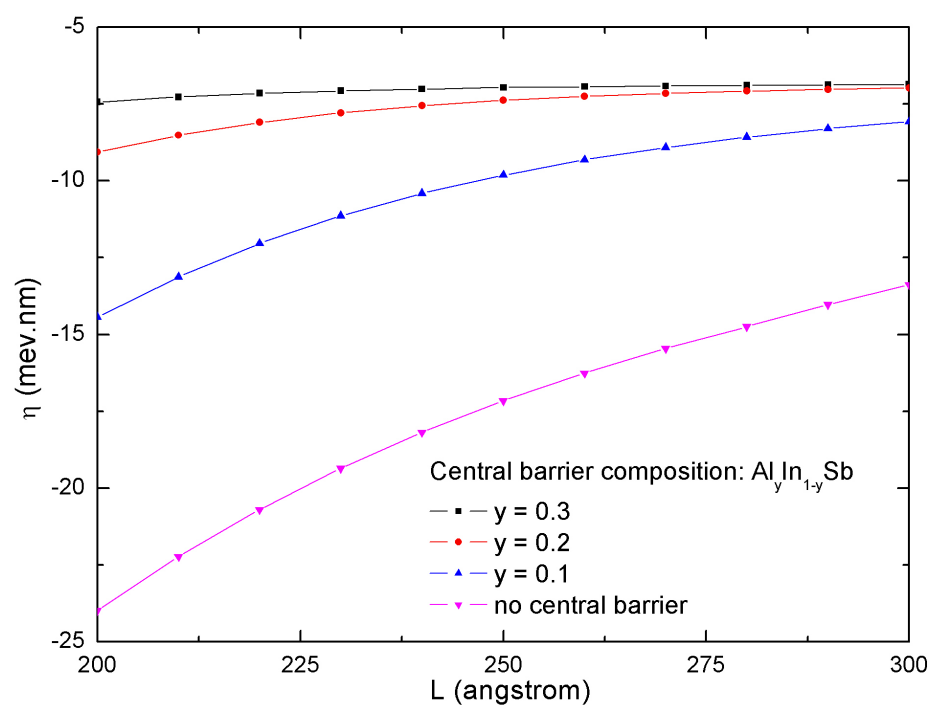

Figura 12: Variação de $\eta$ com relação ao tamanho do poço para três poços quânticos duplos e um simples. A legenda indica a composição da barreira central. Nos casos $y=0.1 \mathrm{e}$ $y=0.2$ temos $V_{b}<V_{0}$, e no caso $y=0.3$ temos $V_{b}=V_{0}$. Para o caso sem barreira central temos um poço simples. Nota-se a diminuição em módulo da constante de acoplamento inter-subbanda conforme o confinamento é maior.

da Fig. 12 (ver equação 3.13). A contribuição para $\eta$ devido ao potencial estrutural do poço é essencialmente proporcional à amplitude da função envelope na borda do próprio poço, uma vez que a derivada desse potencial se aproxima de uma soma de funções delta. Um aumento no tamanho do poço leva a uma menor penetração da função envelope nas barreiras externas, naturalmente reduzindo o valor dessa contribuição em módulo. Essa queda deve ser aproximadamente exponencial, visto que a função de onda na aproximação de elétrons não interagentes tem essa forma funcional na dependência com o tamanho do poço. Nota-se ainda na Fig. 13(b) que a contribuição devido ao potencial de Hartree

Tabela 5: Parâmetros da simulação da figura 12

\begin{tabular}{lrl}
\hline \hline Material & $\mathrm{InSb}$ & \\
$L_{b}$ & 100 & $\AA$ \\
Concentração de Al no Espaçador & 30 & $\%$ \\
Densidade Eletrônica & $1 \times 10^{11}$ & $\mathrm{~cm}^{-2}$ \\
Temperatura & 1.0 & $\mathrm{~K}$ \\
\hline \hline
\end{tabular}




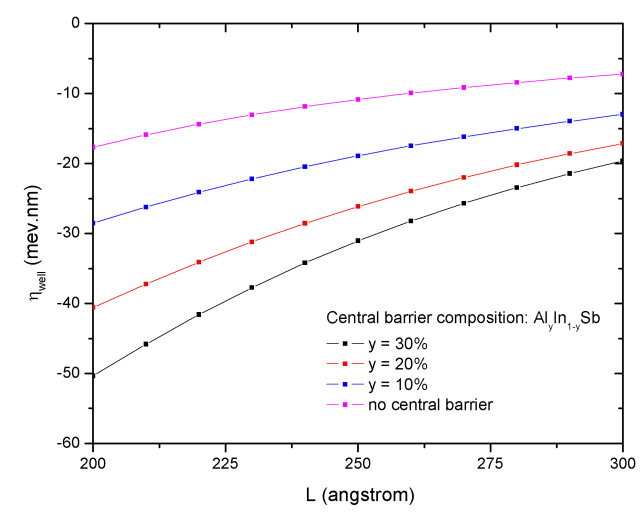

(a) Contribuição devido ao potencial estrutural do (b) Contribuição devido ao potencial autoconsispoço

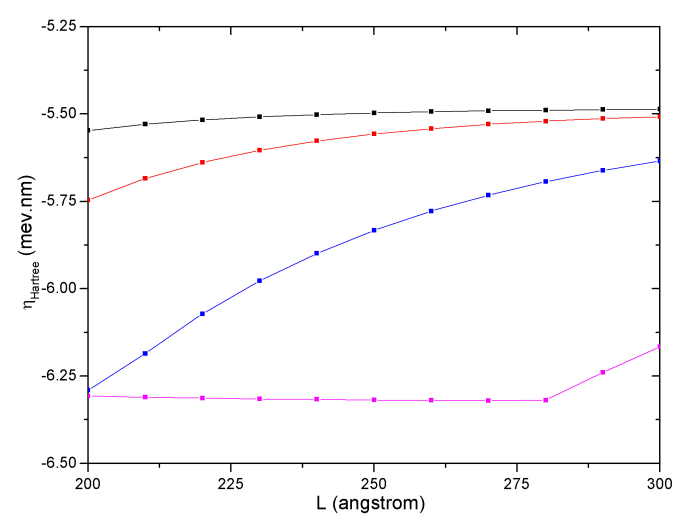

tente (Hartree)

Figura 13: Contribuições a $\eta$ referentes à Fig. 12 devido aos diferentes termos. Nota-se que a escala de variação do termo oriundo do potencial de Hartree é bem menor do que a do termo estrutural.

(elétrons + dopantes) é bem menos sensível à mudanças no tamanho do poço que a parte devido ao potencial estrutural. Apenas uma característica da Fig. 13(b) chama a atenção nesse gráfico: um pequeno salto que acontece em torno de $L=280 \AA$ para a constante de acoplamento do poço sem barreira (poço quântico simples). Naquele ponto o estado correspondente à função de onda ímpar deixa de ser populado, fazendo com que a densidade de carga se concentre mais no centro do poço e levando a um aumento da derivada do potencial de Hartree. Assim, aumenta-se subitamente o ritmo com que essa fração da constante de acoplamento aumenta com relação ao aumento do tamanho do poço.

\subsection{2 $\quad \eta$ vs. densidade bidimensional de portadores}

A Fig. 14 mostra os resultados para $\eta$ em função da densidade areal de portadores dentro do poço quântico. Apresentam-se 3 poços duplos com $L=300 \AA$, 2 poços duplos com $L=200 \AA$, todos com diferentes alturas para as barreiras e, finalmente, um poço simples com $L=300 \AA$. Nota-se a tendência, para uma grande variedade de poços, do valor de $\eta$ crescer em função da densidade areal. Esse comportamento pode ser explicado pelo aumento da amplitude do potencial de Hartree, e portanto dos campos elétricos internos 


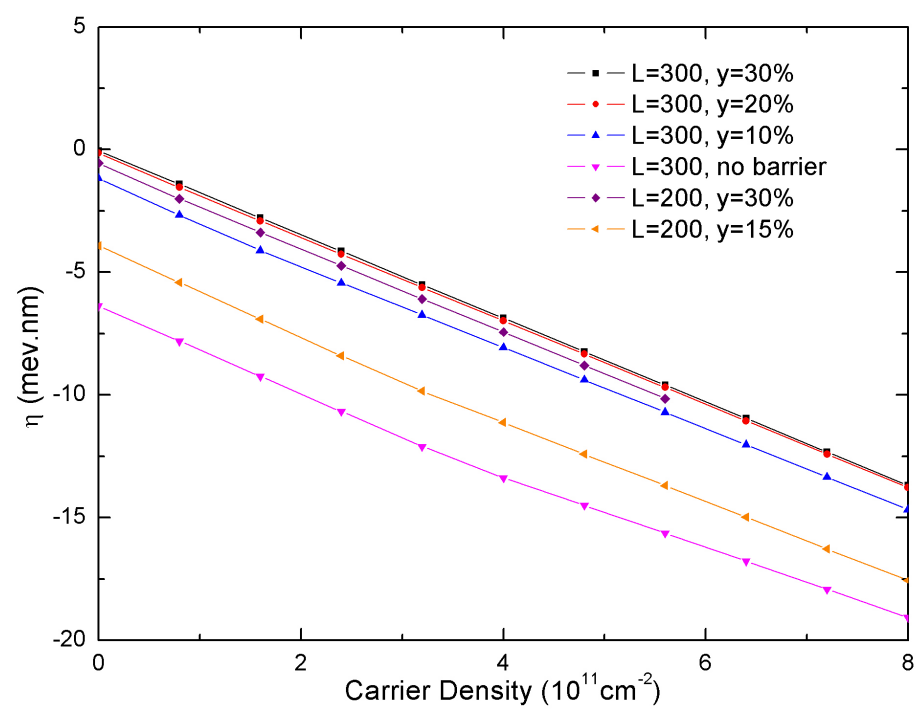

Figura 14: Variação de $\eta$ com relação à densidade de portadores. A legenda indica a composição da barreira central e o tamanho do poço. Nota-se a dependência praticamente linear da constante inter-subbanda com relação à densidade areal de elétrons. O aumento de $\eta$ em módulo deve-se ao aumento da amplitude do potencial autoconsistente provocada pelo aumento da quantidade de carga no poço.

associados a esse potencial ${ }^{3}$. Na Fig. 16, vemos o comportamento de $\eta$ mais detalhada-

Tabela 6: Parâmetros da simulação da Fig. 14

\begin{tabular}{lrl}
\hline \hline Material & InSb & \\
$L_{b}$ & 100 & $\AA$ \\
Concentração de Al no Espaçador & 30 & $\%$ \\
Temperatura & 1.0 & $\mathrm{~K}$ \\
\hline \hline
\end{tabular}

mente para a simulação com $L=300 \AA$ e sem barreira central mostrada na Fig. 14. Na Fig. 16 mostramos também os níveis de energia e o potencial químico, explicitando o valor da densidade de portadores para o qual o potencial químico ultrapassa o valor suficiente para começar a preencher a segunda sub-banda. Nota-se que a dependência entre $\eta$ e a densidade de portadores é muito próxima de uma dependência linear para uma grande gama de geometrias do poço quântico, exceto para o caso da Fig. 16, onde existe uma mudança no coeficiente angular da reta exatamente no ponto onde a segunda sub-banda

\footnotetext{
${ }^{3}$ Note que a condição de contorno é potencial autoconsistente nulo nas bordas do poço.
} 


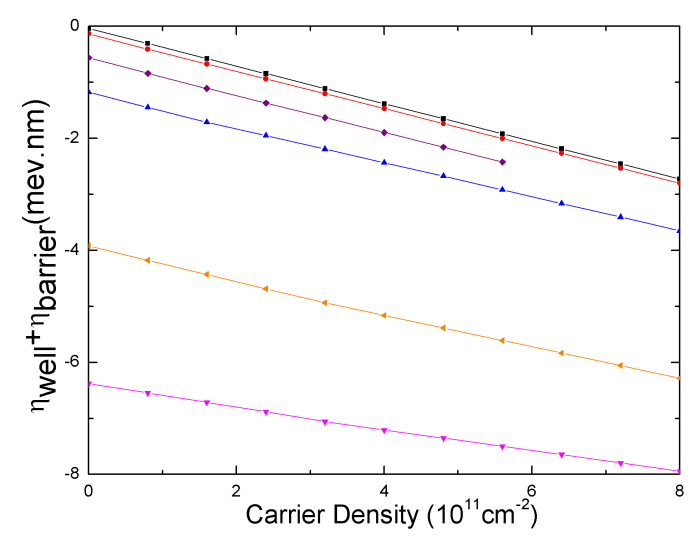

(a) Contribuição devido ao potencial estrutural (poço+barreira)

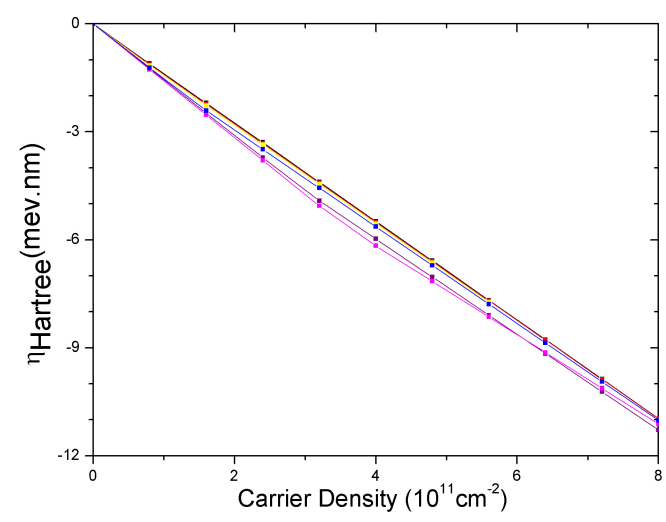

(b) Contribuição devido ao potencial de Hartree

Figura 15: Contribuições a $\eta$ devido aos diferentes termos para os poços quânticos (duplos e simples) da Fig. 14. O código de cores é o mesmo utilizado naquela figura. Nota-se o comportamento aproximadamente linear da constante de acoplamento inter-subbanda em função da densidade. Nota-se ainda que a variação do termo associado ao potencial autoconsistente (Hartree+dopantes) é, desta vez, mais sensível que a do termo estrutural com relação à densidade de portadores.

começa a ser preenchida. O fato pode ser explicado pela diminuição da densidade de cargas no centro do poço conforme a segunda subbanda começa a ser preenchida ${ }^{4}$. Isso leva à diminuição da amplitude do potencial de Hartree no centro do poço, e a uma diminuição dos campos elétricos internos, diminuindo a taxa com que $|\eta|$ cresce com relação a $n_{2 D}$.

\footnotetext{
${ }^{4}$ A função de onda na direção z para a segunda subbanda tem um nó no centro do poço.
} 


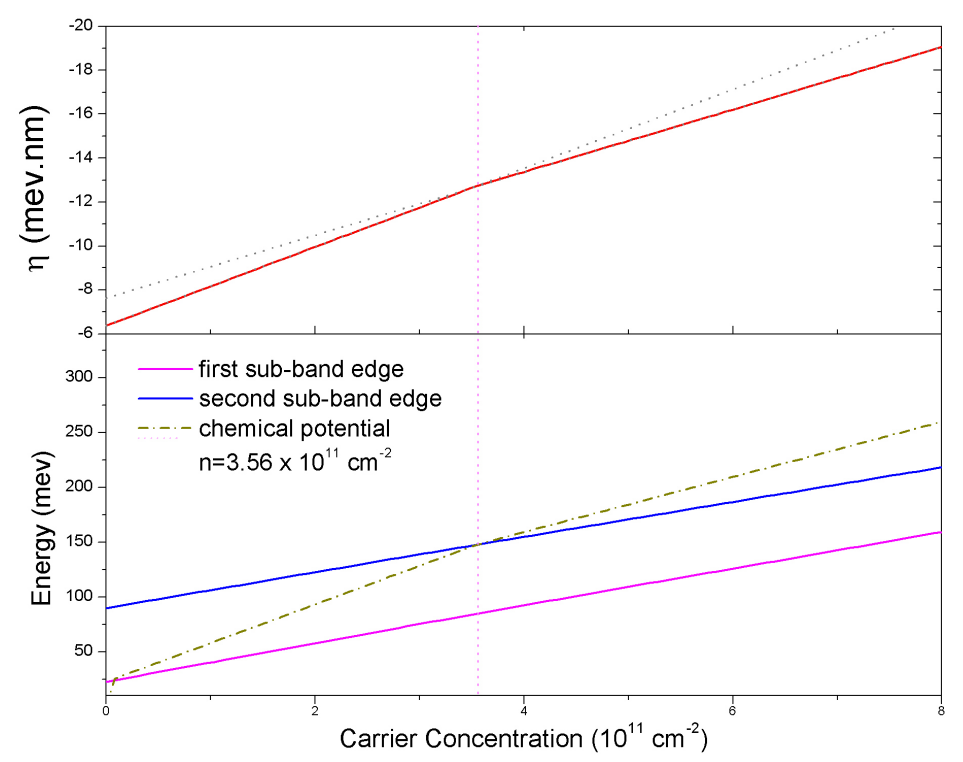

Figura 16: Comparação da variação de $\eta$ e o preenchimento das subbandas. No gráfico superior observa-se $\eta$ vs. $n$. As linhas pontilhadas em azul e magenta são as melhores retas via regressão linear para os dois diferentes coeficientes angulares observados no gráfico. A linha vertical pontilhada indica a densidade para qual a segunda sub-banda começa a ser preenchida $\left(n=3.56 \times 10^{11} \mathrm{~cm}^{-2}\right)$. Na parte inferior observa-se a evolução da energia dos estados fundamental e $1^{\circ}$ excitado e também do potencial químico. O ponto que marca a mudança no coeficiente angular no gráfico superior é exatamente o ponto em que $\mu$ alcança a energia da segunda subbanda e esta começa a ser preenchida com elétrons.

A Fig. 15 apresenta as parcelas correspondentes ao potencial estrutural (poço+barreira) e ao potencial de Hartree separadamente ${ }^{5}$. Nota-se que a contribuição advinda do potencial de Hartree é bem mais sensível à variação da densidade, conforme esperado.

\footnotetext{
${ }^{5} \mathrm{O}$ mesmo código de cores da legenda da Fig. 14 se aplica à Fig.15
} 


\subsection{3 $\eta$ vs. offset estrutural na banda de condução, largura da barreira e altura da barreira}

A Fig. 17 mostra o valor da constante inter-subbanda $\eta$ em função dos parâmetros da barreira, altura e largura. Nota-se que a influência da variação na largura da barreira é tanto maior quando mais "alta" ela for, ou seja, quanto maior a concentração de alumínio na mesma. O efeito contrário, como esperado, também se verifica. A constante diminui em módulo com o aumento da barreira, seja em largura ou altura. O aumento da largura ou da altura da barreira tem efeitos diferentes nas funções envelope do estado fundamental e do primeiro estado excitado e nas diferentes regiões do poço quântico. Nas bordas do poço, a amplitude de ambas as funções envelope aumenta, pois aumenta a penetração da função envelope no espaçador. Isso faz com que $\eta_{\text {poço }}$ aumente em módulo. Na região da borda da barreira, as funções envelope têm comportamentos diferentes: o aumento da barreira faz com que a amplitude da função do estado fundamental diminua na borda da barreira, enquanto a amplitude da função envelope do $1^{\circ}$ estado excitado aumenta nessa mesma região. Esse efeito é exemplificado na Fig. 18 para o caso do aumento da largura da barreira. Uma vez que $\eta_{\text {barreira }}$ é proporcional ao produto das amplitudes de ambas as funções envelopes no ponto correspondente à borda da barreira, a competição entre essas duas variações determinará o comportamento de $\eta_{\text {barreira }}$ contra a largura ou altura da barreira. Nos casos simulados na Fig. 17, $\eta_{\text {barreira }}$ aumenta com relação ao aumento da barreira. Quanto mais alta a barreira, maior a penetração da função envelope na região do espaçador. Isso faz com que $\eta_{\text {poço }}$ aumente em módulo. Essas duas contribuições apresentam, geralmente, sinais opostos. No caso das convenções de sinais usadas para as funções de onda nas simulações aqui apresentadas, em geral temos:

$$
\begin{aligned}
\eta_{\text {poço }} & <0 \\
\eta_{\text {barreira }} & >0 \\
\eta_{\text {Hartree }} & <0
\end{aligned}
$$

A competição entre o aumento em $\eta_{\text {barreira }}$ e $\eta_{\text {poço }}$ determinará o deslocamento de $\eta$ na direção positiva ou negativa. Nos casos apresentados na Fig. 17, a variação em $\eta_{\text {barreira }}$ foi mais determinante e $\eta$ se desloca para o lado positivo. Escolhendo-se uma barreira sufici- 


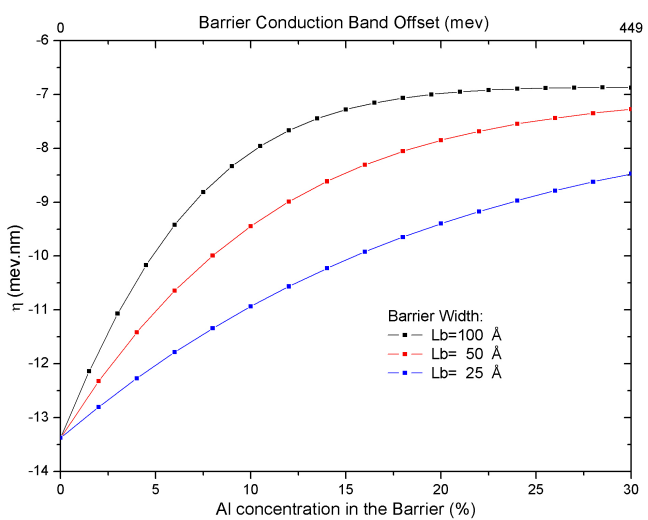

(a) Variação de $\eta$ com relação à concentração de Alumínio na barreira/altura da barreira.

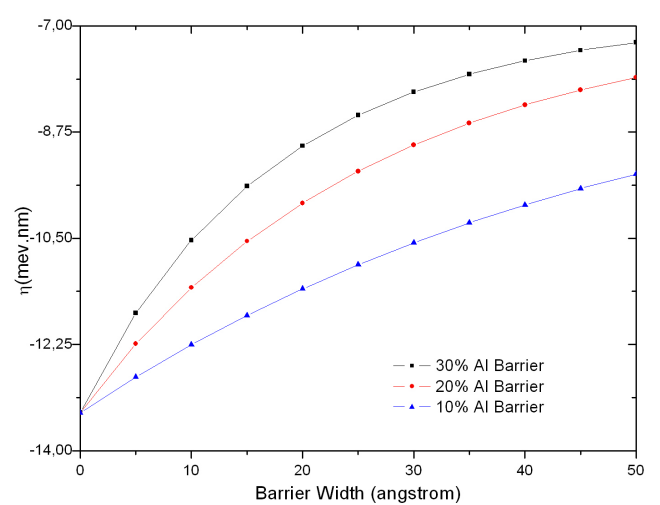

(b) Variação de $\eta$ com relação à largura da barreira central.

Figura 17: Variação de $\eta$ com relação aos parâmetros da barreira: altura e largura. Nota-se que o efeito do alargamento da barreira é tanto maior quanto maior a concentração de $\mathrm{Al}$ e vice-versa. O aumento da altura ou da largura da barreira implica em um aumento de $\eta_{\text {poço }}$ e $\eta_{\text {barreira }}$, contribuições de sinais diferentes. A competição entre essas duas contribuições determina a direção de variação de $\eta$. Nas situações simuladas, a variação de $\eta_{\text {barreira }}$ foi maior.

entemente alta e uma densidade de portadores pequena para que se $\eta_{\text {Hartree }}$ seja pequeno, é possível portanto atingir valores cada vez menores de $\eta$, e até mesmo anulá-lo em alguns casos com uma engenharia adequada dos perfis de potencial. O gráfico da Fig. 19 mostra a variação de $\eta$ com relação ao offset estrutural na banda de condução ${ }^{6}$. Ao aumentar a altura do poço, a função envelope se localiza cada vez mais no centro, diminuindo a largura ocupada por ela e a sua amplitude nas bordas do poço, e aumentando sua amplitude no centro. Isso leva simultaneamente ao aumento de $\eta_{\text {Hartree }}$ e à diminuição de $\eta_{\text {poço }}$. Uma vez que ambas as contribuições são positivas, a competição entre esses dois efeitos produzirá o gráfico da Fig. 19. No caso do poço simples (no central barrier na Fig. 19(a)), a inexistência de $\eta_{\text {barreira }}$ para contrabalançar o aumento de $\eta_{\text {poço }}$ faz com que seja praticamente imperceptível os efeitos de $\eta_{\text {Hartree }}$ Então nesse caso observa-se apenas a diminuição no valor de $\eta$. A Fig. 19(b) mostra o mesmo gráfico sem a curva referente ao poço simples para permitir ver as outras curvas em uma escala mais favorável. Nota-se que há um diminuição de $\eta$ até um valor mínimo e um posterior aumento. Esse comportamento se verifica por causa da competição entre o aumento de $\eta_{\text {Hartree }}$ e a diminuição de $\eta_{\text {poço }}$.

\footnotetext{
6 "Altura do Poço".
} 


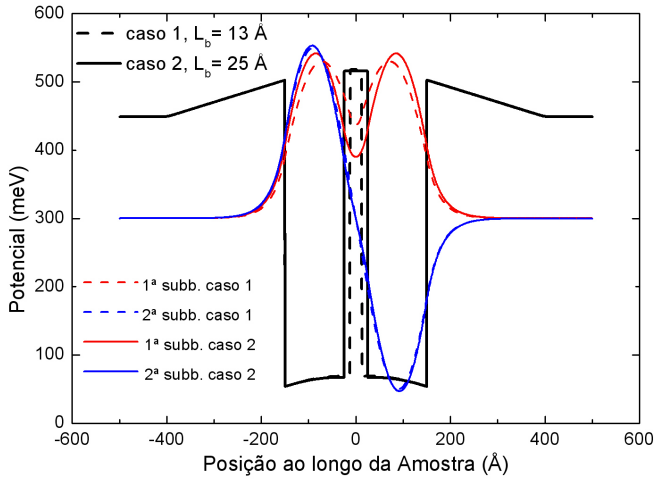

(a) Funções envelope e perfis de potencial.

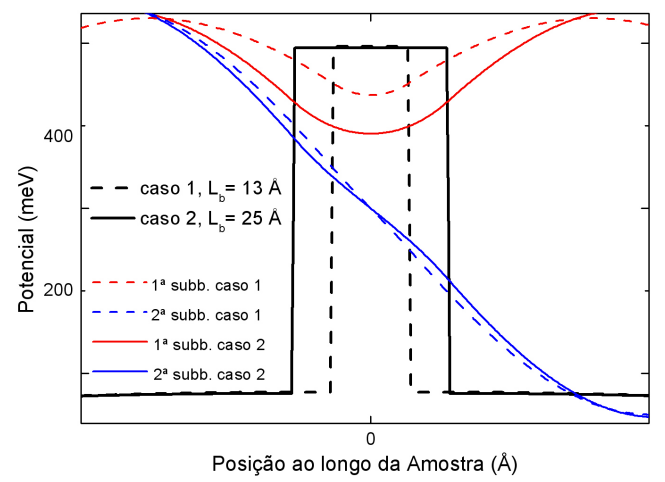

(b) Detalhe da Fig. 18(a) em torno da barreira.

Figura 18: Perfis de potencial e funções envelope para duas situações idênticas porém com barreiras de larguras diferentes. Nota-se que as amplitudes das funções envelope do estado fundamental e do primeiro excitado têm tendências diferentes. A combinação dessas duas variações em direções diferentes determinará a direção da variação de $\eta_{\text {barreira }}$.

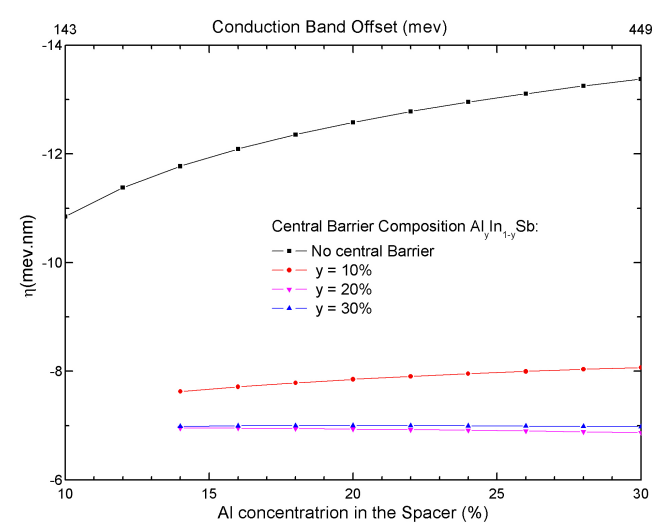

(a) Variação de $\eta$ com relação à concentração de alumínio no espaçador/altura do poço para um poço simples e três poços duplos com diferentes alturas de barreira.

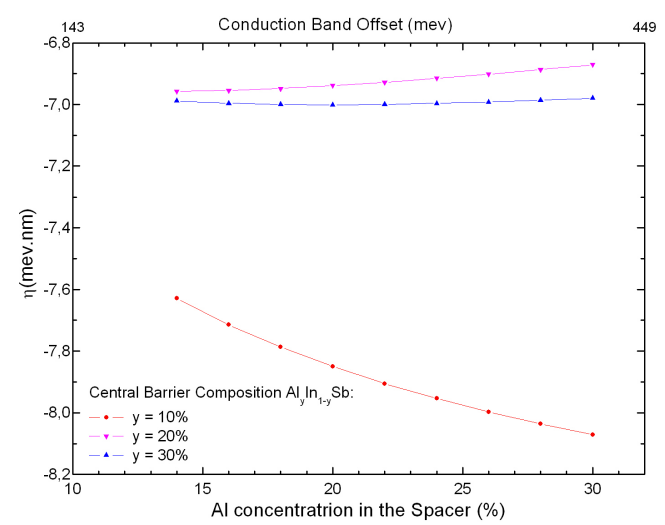

(b) Mesmo gráfico da Fig. 19(a) excluindo a curva referente ao poço simples (no central barrier)

Figura 19: Variação de $\eta$ com relação à concentração de alumínio no espaçador/altura do poço. A competição entre o aumento de $\eta_{\text {Hartree }}$ e a diminuição de $\eta_{\text {poço }}$ provoca a existência de um ligeiro mínimo em $\eta$. 


\subsubsection{Variando o Material do Poço}

Para exemplificar o efeito de parâmetros da estrutura de bandas sobre o valor de $\eta$, apresentamos nas figuras abaixo resultados para poços de ligas AlGaAs/GaAs/AlGaAs. Por possuir um gap bem maior e uma energia de split-off substancialmente menor que a do InSb, os resultados para a constante de acoplamento para o GaAs são em torno de duas ordens de grandeza menores do que os do InSb. Na Fig. 20(a), é apresentado um gráfico de $\eta$ versus a densidade de portadores. Nota-se o mesmo comportamento já antes verificado para o InSb, linear com eventuais mudanças de coeficiente angular quando a segunda sub-banda passa a ser preenchida. Na Fig. 20(b), apresenta-se um gráfico de $\eta$

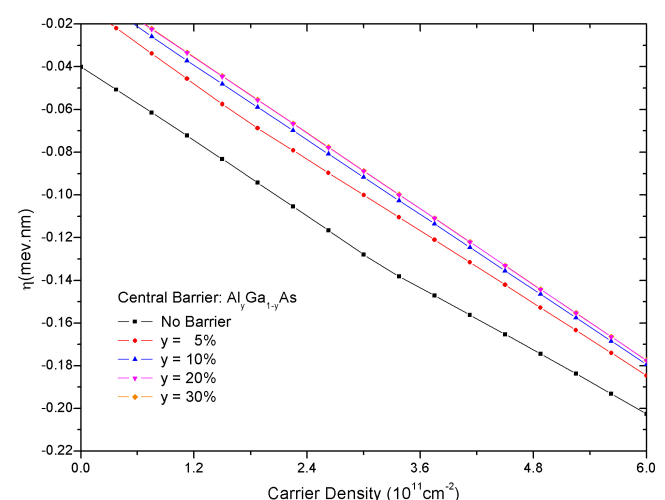

(a) Gráfico de $\eta$ contra a densidade areal de por- (b) Variação de $\eta$ com relação à concentração de tadores.

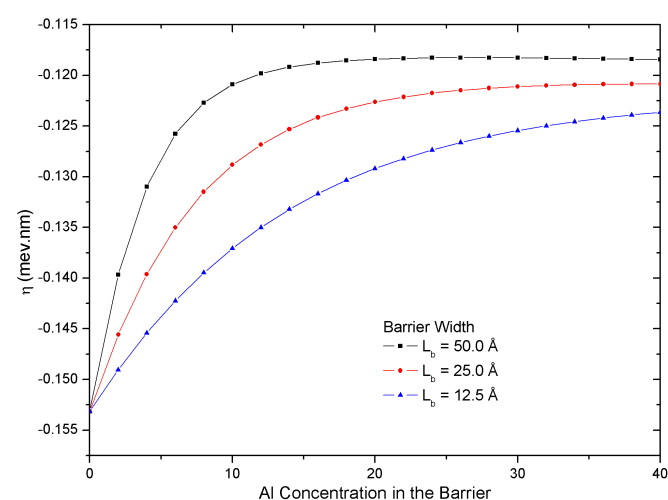

Al na barreira para poços feitos de GaAs.

Figura 20: Gráficos de $\eta$ vs. altura da barreira central e densidade areal de portadores em poços de GaAs. Nota-se um comportamento similar aos dos poços de InSb, com uma diminuição em fatores de até ordem 100 no valor de $\eta$.

contra a altura da barreira central (concentração de Al na barreira) também para poços de GaAs. Os valores da constante inter-subbanda calculados até agora estão na mesma ordem de grandeza dos experimentalmente medidos para a constante de Rashba[3, 4].

\subsection{Poços não simétricos e constante de Rashba}

Simulamos também poços originalmente simétricos submetidos a um campo elétrico externo através de eletrodos. A disposição dos eletrodos está representada na Fig.21. A 


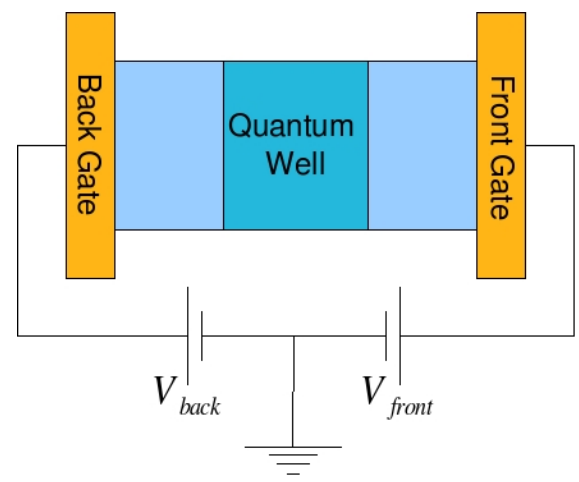

Figura 21: Descrição da posição dos eletrodos relativamente aos poços quânticos simulados. As voltagens aplicadas no front e noback gate foram variadas e foram determinadas a constante inter-subbanda e as constantes de Rashba associadas a cada sub-banda.

quebra da simetria de inversão devido a esses campos elétricos faz com que as constantes de Rashba ${ }^{7}$ não sejam mais iguais a zero. O deslocamento do perfil do poço com relação ao potencial químico de um reservatório externo permite controlar a densidade areal de portadores no poço. Nas Fig. 22 e 23, são mostrados os valores das constantes de acoplamento intersubbanda e de Rashba para as duas subbandas em função do potencial elétrico externo. Em ambas as figuras, o potencial do back gate foi escolhido como $100 \mathrm{mV}$ e o front gate foi variado de 0 até $200 \mathrm{mV}$. O potencial químico do reservatório foi escolhido de tal forma que a carga total do poço (regiões dopadas depletadas + elétrons dentro do poço) fosse nula na situação simétrica. Na Fig. 22 temos um poço sem barreira central e na Fig. 23 um poço idêntico ao primeiro a menos de uma barreira central. Nas duas figuras pode-se ver claramente que as constantes de Rashba se tornam nulas na situação simétrica (front gate $=$ back gate) e podem ser ajustadas através da aplicação de um campo elétrico externo - fato que já foi experimentalmente determinado. A constante de acoplamento inter-subbanda também responde à variação do campo elétrico externo, aproximadamente na mesma escala de variação que as constantes de Rashba.

\footnotetext{
${ }^{7}$ Para uma e outra sub-banda
} 


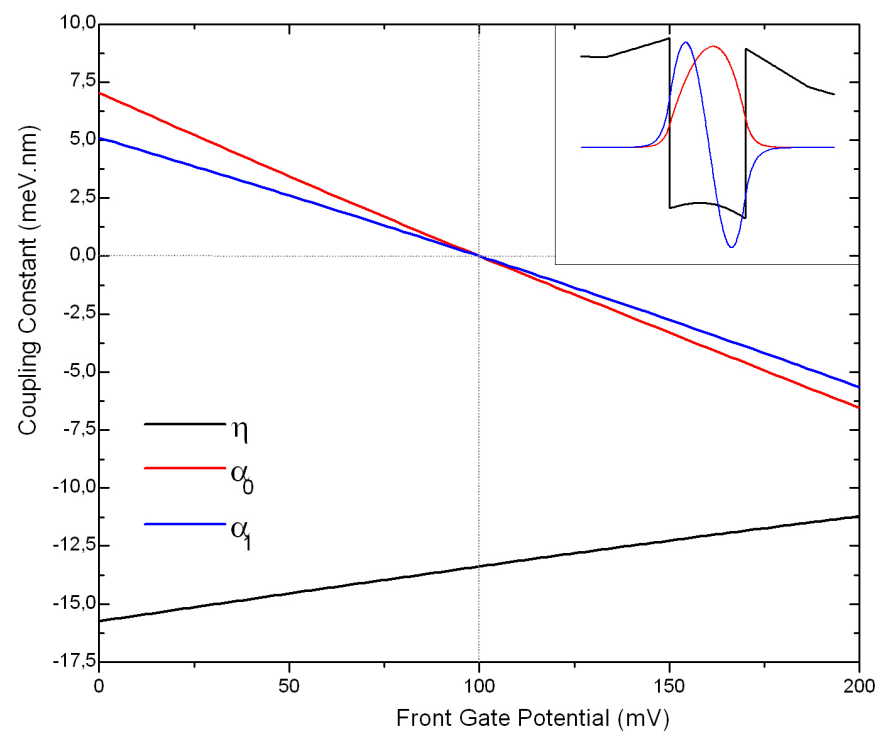

Figura 22: Constantes de Acoplamento em um poço quântico duplo em função do potencial elétrico no front gate para um potencial de back gate de $100 \mathrm{mV}$. Nota-se, como antes, um comportamento aproximadamente linear das constantes de acoplamento com relação ao potencial dos eletrodo.

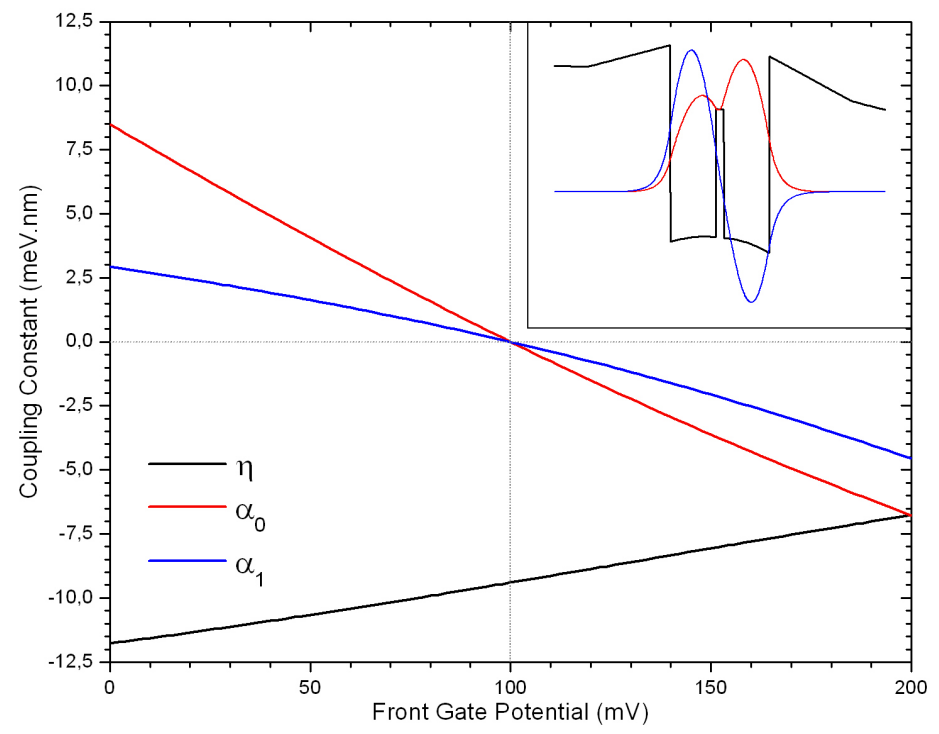

Figura 23: Constantes de Acoplamento em um poço quântico simples em função do potencial elétrico no front gate para um potencial de back gate de $100 \mathrm{mV}$ para um poço com uma barreira central.Nota-se que a ordem de grandeza das constantes é similar, e parecidas com as das referências $[3,4]$. Nota-se ainda o comportamento aproximadamente linear com relação ao potencial do eletrodo. 


\subsection{Massas efetivas}

Como visto na página 51, para pequenos valores da constante de acoplamento intersubbanda, seu efeito pode ser visto como uma redefinição das massas efetivas para cada sub-banda dada pela Eq.(3.15). A razão entre a diferença das massas efetivas nas subbandas devido a esse efeito e a massa efetiva do material é dada por:

$$
\frac{\Delta m^{*}}{m^{*}}=\frac{4 \epsilon_{S O}}{\epsilon_{-}}
$$

Na Fig. 24, este cálculo foi feito para os sistemas das Figs. 22 e 23. Nota-se que o efeito é pequeno para a maioria das estruturas observadas. Entretanto alguns sistemas particulares

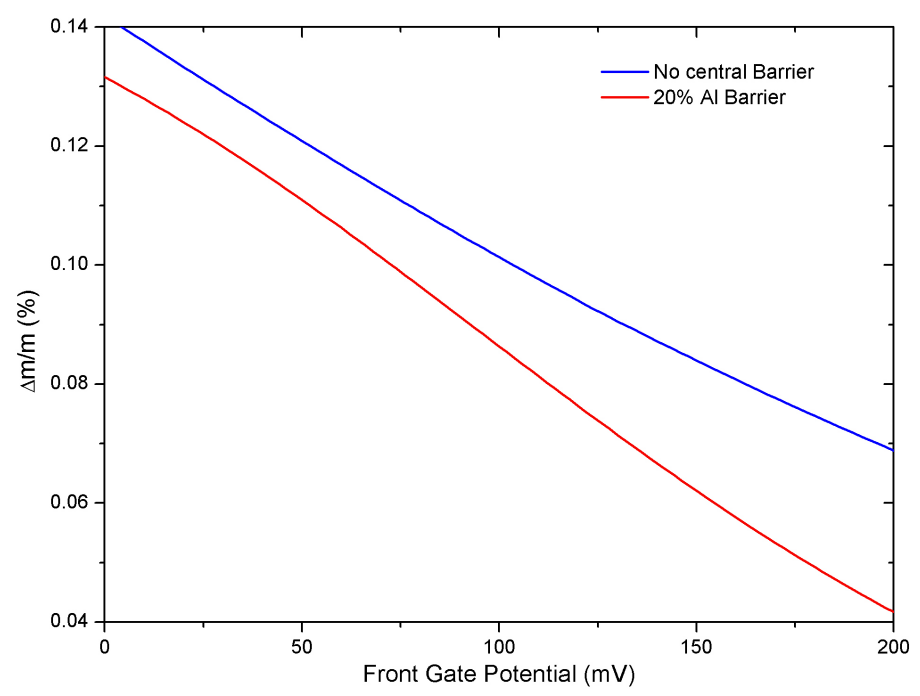

Figura 24: Razão entre a diferença das massas efetivas nas subbandas e a massa efetiva do material para as simulações das Figs. 22 e 23. A diminuição em $\eta$ observada no gráfico contra a diferença de potencial leva a uma diminuição ainda mais acentuada na diferença entre as massas efetivas que é proporcional à $\eta^{2}$.

fogem dessa regra. Na Fig. 25 apresenta-se um sistema cujo resultado para a renormalização de massa ultrapassa os 3\%. Na referência [1], Bernardes et al.[1] mostram cálculos que resultaram numa renormalização da ordem de 5\%. Esses resultados são possíveis em estruturas em que a diferença de energia entre os fundos das duas subbandas (chamada no texto acima de $\epsilon_{-}$) é muito pequena, da ordem de $1 \mathrm{meV}$, ou menores. Assim, a razão 
descrita na Eq. 3.15 é maximizada. Para diminuir $\epsilon_{-}$pode-se aumentar a largura ou altura
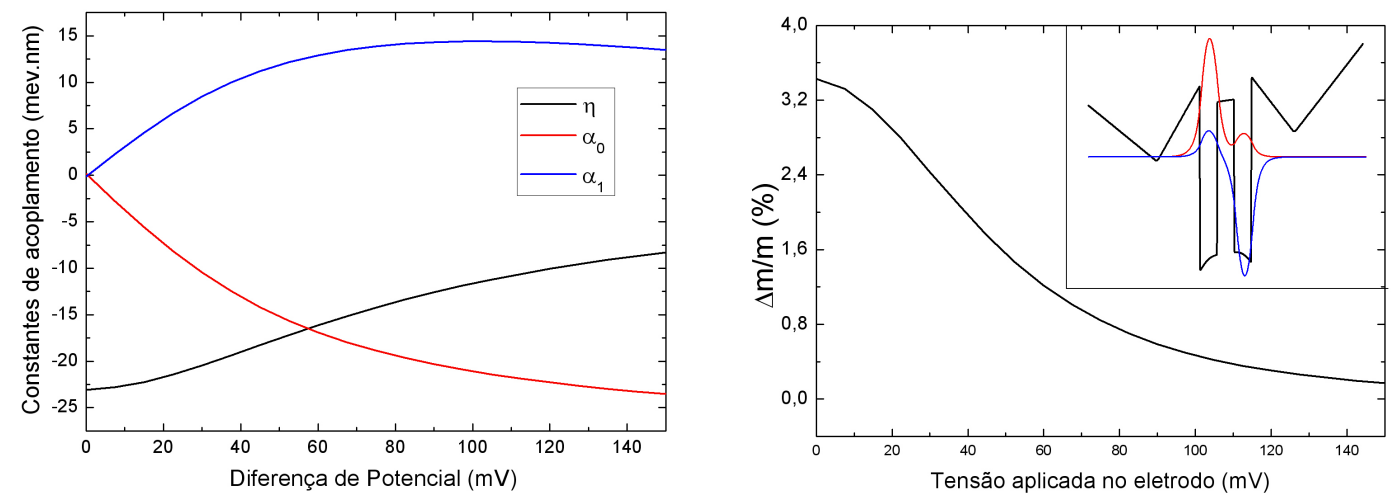

(a) Valores das constantes de acoplamento de (b) Razão entre a diferença das massas efetivas Rashba para ambas as subbandas e da constante nas subbandas e a massa efetiva bulk inter-subbanda.

Figura 25: Cálculo das constantes de acoplamento de Rashba e inter-subbanda e da razão entre as massas das diferentes subbandas para um poço quântico duplo sujeito a um potencial externo. A estrutura em questão não é neutra na situação simétrica e possui uma densidade de doadores na região dopada da ordem de 10 vezes maior do que as simuladas anteriormente graças e uma densidade de elétrons da mesma ordem de grandeza. Nota-se um efeito de renormalização de massa da ordem de 3\% a $4 \%$ na situação simétrica e uma grande faixa de variação para as constantes de acoplamento em função da tensão do gate.

da barreira, isolando mais os poços entre si, ou aumentar a densidade de portadores ou a constante dielétrica, elevando assim a amplitude do potencial de Hartree e causando um efeito similar. 


\section{Conclusões}

Fizemos o cálculo autoconsistente da constante de acomplamento spin-órbita intersubbanda e da constante de Rashba para uma grande gama de poços quânticos duplos e simples variando diversos parâmetros estruturais, materiais diferentes, densidade de portadores e potenciais elétricos aplicados. O modelo usado para escrever a expressão das constantes de acoplamento é o modelo de Kane $8 \times 8$ tradicional para estruturas do tipo zincblende através do qual se obteve, via Folding Down, um hamiltoneano efetivo para a banda de condução que contém termos de acoplamento spin-órbita inter-subbanda e de Rashba. Nas expressões obtidas é interessante observar que o acoplamento spin-órbita na banda de condução é proporcional a uma espécie de campo elétrico médio proveniente das bandas de valência, dirimindo portanto a questão da objeção de Ando[28, 30, 31]. O fato das forças médias serem nulas em um estado ligado não implica em constantes de acoplamento nula ainda que não se considere o efeito das massas efetivas diferentes ao longo da amostra, uma vez que o campo elétrico médio relevante para o caso é proveniente das bandas de valência e não da própria banda de condução. Esse fato, já discutido na literatura para a constante de Rashba, é reforçado pela dedução teórica aqui exposta e se aplica também à constante de acoplamento inter-subbanda.

Os resultados dos cálculos autoconsistentes indicam que a magnitude da interação spin-órbita inter-subbanda deve ser da mesma ordem de grandeza da interação Rashba já conhecida. Seus efeitos sobre a relação de dispersão são mais sutis - por exemplo não quebra a degenerescência de spin e o efeito mais visível é uma alteração das massas efetivas nas diferentes subbandas bastante modesta na maioria das estruturas. Tendo isso em mente, deve ser mais difícil de observar experimentalmente os efeitos desse novo acoplamento. Entretanto existem regimes em que a renormalização das massas efetivas é substancial, o 
que deve ter um efeito experimental observável. A possibilidade de se fazer medidas resolvidas em subbandas [65] aumenta as chances de que hajam efeitos que possam identificar um possível acoplamento inter-subbanda. Outra via para a possível medida experimental desse acoplamento é a condutividade Hall de spin, calculada na referência [1]. Os valores numéricos para as constantes de acoplamento apresentados neste trabalho serão úteis na determinação da intensidade da condutividade Hall de spin e também do Zitterbewegung não usual relatado também em [1].

O valor da constante de acoplamento calculado varia substancialmente com a densidade de portadores, podendo ser mesmo anulada, controlando-se a densidade de portadores através da aplicação de campos elétricos externos. A faixa de variação da constante de acoplamento inter-subbanda é a mesma da constante de Rashba, sendo possível controlá-la experimentalmente e mesmo anulá-la em alguns casos. O mesmo ocorre para o efeito de renormalização das massas efetivas, sendo possível variá-lo de virtualmente zero até valores em torno de 3\%-4\% nos cálculos realizados aqui e até 5\% em alguns casos mais extremos.

Nota-se ainda que a influência potencial autoconsistente fornece uma parcela substancial do valor das constante de acoplamento inter-subbanda e de Rashba. Entretanto não é possível prever de antemão que efeitos possivelmente surgiriam com a inclusão de efeitos de troca e correlação em sistemas que apresentam ferromagnetismo de efeito Hall ou outras situações em que os efeitos do princípio de Pauli nos portadores são importantes. Por essa razão sugerimos como possível direção de expansão dos cálculos aqui realizados a implementação de um cálculo via Teoria do Funcional Densidade, aplicada recentemente no contexto de heteroestruturas semicondutoras nas referências $[57,58]$. Esta direção é importante tanto na determinação da influência desses efeitos no valor das constantes de acoplamento quanto na necessidade de entender os efeitos da interação spin-órbita nesses sistemas ${ }^{1}$. Os resultados apresentados aqui sugerem que é essencial que a determinação das constantes de interação spin-órbita seja feita de forma autoconsistente.

\footnotetext{
${ }^{1} \mathrm{O}$ interplay entre interação de troca e acoplamento Rashba foi recentemente estudado experimentalmente em [66]
} 


\section{Referências}

1 BERNARDES, E. S. et al. Spin-orbit interaction in symmetric wells with two subbands. Physical Review Letters, v. 99, n. 7, p. 076603, 2007.

2 BERNARDES, E. S. et al. Spin orbit interaction and zitterbewegung in symmetric wells. Phys. Stat. Sol. (c), v. 3, n. 12, p. 4330-4333, 2007.

3 KOGA, T.; NITTA, J.; VEENHUIZEN, M. van. Ballistic spin interferometer using the rashba effect. Phys. Rev. B, v. 70, n. 3, p. 161302, 2004.

4 HU, C.-M. et al. Zero-field spin splitting in an inverted $I n_{0.53} G a_{0.47} A s / \operatorname{In}_{0.52} A l_{0.48} A s$ heterostructure: Band nonparabolicity influence and the subband dependence. Phys. Rev. $B$, v. 60, n. 11, p. $7736-7739,1999$.

5 KLitZING, K. von. The quantized hall effect. Rev. Mod. Phys., v. 58, n. 3, p. 519-531, 1986.

6 TSUI, D. C.; STORMER, H. L.; GOSSARD, A. C. Two-dimensional magnetotransport in the extreme quantum limit. Phys. Rev. Lett., v. 48, n. 22, p. 1559-1562, 1982.

7 KATO, Y. K. et al. Observation of the spin hall effect in semiconductors. Science, v. 306, p. 1910, 2004.

8 D'AMICO, I.; VIGNALE, G. Theory of spin coulomb drag in spin-polarized transport. Phys. Rev. B, v. 62, n. 8, p. 4853-4857, 2000.

9 KROEMER, H. The thomas precession factor in spin-orbit interaction. Am. J. Phys., v. 72, n. 1, p. 51.

10 SAKURAI, J. J. Advanced Quantum Mechanics. Menlo Park, California: Addison-Wesley.

11 FOLDY, L. L.; WOUTHUYSEN, S. A. On the Dirac theory of spin 1/2 particles and its non-relativistic limit. Phys. Rev., v. 78, n. 1, p. 29-36, 1950.

12 ZAWADZKI, W. On the $v^{2} / c^{2}$ expansion of the Dirac equation with external potentials. American Journal of Physics, v. 73, n. 8, p. 756-758, 2005.

13 DRESSELHAUS, G. Spin-orbit coupling effects in Zinc Blende structures. Phys. Rev., v. 100, n. 2, p. 580-586, 1955. 
14 JANCU, J. et al. Atomistic spin-orbit coupling and $\mathrm{k} \cdot \mathrm{p}$ in III-V semiconductors. Journal of Superconductivity: incorporating novel magnetism, v. 16, p. 237, 2003.

15 RASHBA, E. I.; Y.A.BYCHKOV. Sov. Phys. Solid State, v. 2, p. 1109, 1960.

16 BYCHKOV, Y. A.; RASHBA, E. I. Oscillatory effects and the magnetic susceptibility of carriers in inversion layers. J. Phys. C: solid state physics, v. 17, n. 33, p. 6039, 1984.

17 SILVA, E. A. A. e; ROCCA, G. C. L.; BASSANI, F. Spin-orbit splitting of electronic states in semiconductor asymmetric quantum wells. Phys. Rev. B, v. 55, p. 16293, 1997.

18 SILVA, E. A. de Andrada e. Conduction-subband anisotropic spin splitting in iii-v semiconductor heterojunctions. Phys. Rev. B, v. 46, n. 3, p. 1921-1924, 1992.

19 ERLINGSSON, S.; EGUES, J. C.; LOSS, D. Spin densities in parabolic quantum wires with rashba spin-orbit interaction. Phys. Stat. Sol. (c), v. 3, n. 12, p. 4317, 2006.

20 GREINER, W. Relativistic Quantum Mechanics: Wave Equations. New York: Springer Verlag, 2000.

21 DATTA, S.; DAS, B. Electronic analog of the electro-optic modulator. Appl. Phys. Lett., v. 56, n. 7, p. 665, 1990.

22 SCHLIEMANN, J.; EGUES, J. C.; LOSS, D. Nonballistic spin-field-effect transistor. Phys. Rev. Lett., v. 90, n. 14, p. 146801, 2003.

23 KOGA, T.; SEKINE, Y.; NITTA, J. Experimental realization of a ballistic spin interferometer based on the rashba effect using a nanolithographic defined square loop array. Phys. Rev. B, v. 74, n. 4, p. 041302, 2006.

24 BURKARD, G.; EGUES, J. C.; LOSS, D. Noise of spin-polarized currents at a beam splitter with local spin-orbit interaction. Journal of Superconductivity: incorporating novel magnetism, v. 16, n. 2, p. 237, 2003.

25 KOGA, T.; NITTA, J.; TAKAYANAGI, H. Spin-filter device based on the rashba effect using a nonmagnetic resonant tunneling diode. Phys. Rev. Lett., v. 88, n. 12, p. 126601, 2002.

26 WOLF, S. et al. Spintronics: A spin-based electronics vision for the future. Science, v. 294, n. 5546, p. 1488, 2001.

27 DARR, A.; KOTTHAYS, J.; ANDO, T. In: FUMI, F. G. (Ed.). Proceedings of the 13th International Conference on the Physics of Semiconductors, Rome, Italy, 1976. Amsterdaltl: North Holland, 1977. p. 774-778.

28 ZAWADZKI, W.; PFEFFER, P. Average forces in bound states and resonant quantum states. Phys. Rev. B, v. 64, n. 23, p. 235313, 2001. 
29 ZAWADZKI, W.; PFEFFER, P. Spin splitting of subband energies due to inversion asymmetry in semiconductor heterostructures. Semicond. Sci. Technol., v. 19, p. R1, 2004 .

30 WINKLER, R. Rashba spin splitting and Ehrenfest's theorem. Physica E, v. 22, p. $450,2004$.

31 SILVA, E. A. de Andrada e; ROCCA, G. C. L. Spin-orbit splitting in the conduction subband of semiconductor asymmetric heterostructures. Brazilian Journal of Physics, v. 26, n. 1, p. 144, 1996.

32 WINKLER, R. Spin-Orbit Coupling Effects in Two-Dimensional Electron and Hole Systems. Berlin/Heildelberg: Springer, 2003.

33 ZAWADZKI, W. Zitterbewegung and its effects on electrons in semiconductors. Phys. Rev. B, v. 72, p. 085217, 2005.

34 PFEFFER, P. Effect of inversion asymmetry on the conduction subbands in GaAs $-\mathrm{Ga}_{1-\mathrm{x}} \mathrm{Al}_{\mathrm{x}} \mathrm{As}$ heterostructures. Phys. Rev. B, v. 59, p. 15902, 1999.

35 KAINZ, J.; RöSSLER, U.; WINKLER, R. Anisotropic spin splitting and spin relaxation in algaas/gaas quantum structures. Journal of Superconductivity: Incorporating Novel Magnetism, v. 16, p. 323, 2003.

36 WINKLER, R. Rashba spin splitting in two-dimensional electron and hole systems. Phys. Rev. B, v. 62, p. 4245, 2000.

37 KAINZ, J.; RöSSLER, U.; WINKLER, R. Anisotropic spin splitting and spin relaxation in asymmetric zinc blende semiconductor quantum structures. Phys. Rev. Lett., v. 68, p. $075322,2003$.

38 HASEGAWA, M. M.; SILVA, E. A. de Andrada e. Spin-orbit-split subbands in IV-VI asymmetric quantum wells. Phys. Rev. B, v. 68, n. 20, p. 205309, 2003.

39 LITVINOV, V. I. Electron spin splitting in polarization-doped group-III nitrides. Phys. Rev. B, v. 68, p. 155314, 2003.

40 ARAÚJO, C. M.; SILVA, A. F. da; SILVA, E. A. de Andrada e. Electron spin $ّ$ orbit split minibands in semiconductor asymmetric superlattices. Phys. Rev. B, v. 65, n. 23, p. 235305, 2002.

41 SILVA, E. A. de Andrada e; ROCCA, G. C. L. Rashba spin splitting in semiconductor quantum wires. Phys. Rev. B, v. 67, n. 16, p. 165318, 2003.

42 NITTA, J.; AKAZAKI, T.; TAKAYANAGI, H. Gate control of spin-orbit in an inverted $\mathrm{In}_{0.53} \mathrm{Ga}_{0.47} \mathrm{As} / \mathrm{In}_{0.52} \mathrm{Ga}_{0.48}$ As heteroestructure. Phys. Rev. Lett., v. 78, p. 1335, 1997. 
43 ENGELS, G. et al. Experimental and theoretical approach to spin splitting in modulation-doped $\mathrm{In}_{\mathrm{x}} \mathrm{Ga}_{1-\mathrm{x}} \mathrm{As} / \mathrm{InP}$ quantum wells for $\mathrm{b} \rightarrow 0$. Phys. Rev. B, v. 55, p. R1958, 1997.

44 KOGA, T. et al. Rashba spin-orbit coupling probed by the weak antilocalization analysis in InAlAs/InGaAs/InAlAs quantum wells as a function of quantum well asymmetry. Phys. Rev. Lett., v. 89, p. 046801, 2002.

45 KOGA, T.; NITTA, J.; MARCET, S. Structural control of rashba spin-orbit coupling in $\mathrm{In}_{0.52} \mathrm{Ga}_{0.48} \mathrm{As} / \mathrm{In}_{0.53} \mathrm{Ga}_{0.47} \mathrm{As} / \operatorname{In}_{0.52} G a_{0.48}$ As quantum wells. Journal of Superconductivity: Incorporating Novel Magnetism, v. 16, p. 331, 2003.

46 STERN, F. Self-consistent results for n-type si inversion layers. Phys. Rev. B, v. 5, p. $4891,1972$.

47 VINTER, B. Subbands in back-gated heterojunctions. Solid State Communications, v. 48 , p. 151, 1983.

48 BASTARD, G. Wave Mechanics Applied to Semiconductor Heterostructures. Les Ulis Cedex: Les Èditions de Physique, 1988.

49 ASHCROFT, N. W.; MERMIN, N. D. Solid State Physics. New York: Saunders College Publishing, 1976.

50 YU, P. Y.; CARDONA, M. Fundamentals of semiconductors: physics and materials properties. Berlin: Springer, 2005.

51 KAnE, E. O. Band structure of indium antimonide. J. Phys. Chem. Solids., v. 1, p. 249-261, 1957.

52 COHEN-TANNOUDJI, C.; DIU, B.; LALOE, F. Quantum Mechanics. New York: John Wiley \& Sons, 1977.

53 VURGAFTMAN, I.; MEYER, J. R.; RAM-MOHAN, L. R. Band parameters for III-V compound semiconductors and their alloys. J. Appl. Phys., v. 89, n. 11, p. 5815-5875, 2001.

54 SILVA, E. A. de Andrada e; ROCCA, G. C. L.; BASSANI, F. Spin-split subbands and magneto-oscillations in iii-v asymmetric heterostructures. Phys. Rev. B, v. 50, n. 12, p. 8523-8533, 1994.

55 ZAWADZKI, W.; PFEFFER, P. Bychkov-rashba spin splitting and its dependence on magnetic field in $\mathrm{InSb} / \mathrm{In}_{0.91} \mathrm{Al}_{0.09} \mathrm{Sb}$ asymmetric quantum wells. Phys. Rev. B, v. 68, p. 035315, 2003.

56 ZAWADZKI, W.; PFEFFER, P. Influence of barrier material on spin splitting due to inversion asymmetry in heterostructures. Journal of Superconductivity: Incorporating Novel Magnetism, v. 16, p. 351, 2003. 
57 FREIRE, H. J. P.; EGUES, J. C. Hysteretic resistance spikes in quantum hall ferromagnets without domains. Phys. Rev. Lett., v. 99, n. 2, p. 026801, 2007.

58 FERREIRA, G. J.; FREIRE, H. J.; EGUES, J. Ringlike structures in the densitymagnetic-field $\rho_{x x}$ diagram of two-subband quantum hall systems. Phys. Stat. Sol. (c), v. 3, p. 4364, 2006.

59 MERZBACHER. Quantum Mechanics. 2nd.. ed. [S.l.]: John Wiley \& Sons, Inc., 1998.

60 GIGLBERGER, S. et al. Rashba and dresselhaus spin splittings in semiconductor quantum wells measured by spin photocurrents. Phys. Rev. B, v. 75, n. 3, p. 035327, 2007.

61 GANICHEV, S. D. et al. Experimental separation of rashba and dresselhaus spin splittings in semiconductor quantum wells. Phys. Rev. Lett., v. 92, p. 256601, 2004.

62 BERNARDES, E. O Método Numérico Numerov Via Computação Algébrica. Disponivel em: 〈www.lia.if.sc.usp.br〉. Acesso em 20/07/2006.

63 BERNARDES, E. A direct numerov sixth order numerical scheme to accurately solve the unidimensional poisson equation with dirichlet boundary conditions. [mensagem pessoal] Mensagem recebida por〈rafael.calsaverini@gmail.com〉 em 10/01/2007.

64 PRESS, W. H. et al. Numerical Recipes in Fortran 77\%: The art of scientific computing. New York: Cambridge University Press, 1992.

65 FLETCHER, R. et al. Two-band electron transport in a double quantum well. Phys. Rev. B, v. 71, p. 155310, 2005.

66 LIU, J. et al. Competition between rashba effect and exchange interaction in a $\mathrm{Hg}_{0.98} \mathrm{Mn}_{0.02}$ Te magnetic 2DEG. Journal of Superconductivity: Incorporating Novel Magnetism, v. 16, p. 365, 2003. 


\section{APÊENICE A - Digressão sobre integrais de produtos de funções que variam em escalas diferentes}

Na seção 2.4, encontramos, na dedução da Aproximação da Função Envelope, diversas integrais do tipo:

$$
I=\int f(\vec{x}) g(\vec{x}) d^{3} x
$$

em que a função $f(\vec{x})$ varia lentamente, ou seja, varia pouco na escala do parâmetro de rede do crista, e a função $g(\vec{x})$ varia rapidamente: tem a periodicidade da rede de Bravais. Neste apêndice desenvolveremos uma aproximação útil[48] nesses casos que foi usada no cálculo dos elementos de matriz da equação (2.22).

Toda função periódica pode ser expandida numa série de Fourier ${ }^{1}$ :

$$
g(\vec{x})=\sum_{\vec{K} \in \text { R.R. }} e^{i \vec{K} \cdot \vec{x}} \tilde{g}(\vec{K}),
$$

onde o vetor $\vec{K}$ percorre os vetores da rede recíproca ${ }^{2}$. Substituindo essa série na integral original:

$$
I=\sum_{\vec{K} \in \text { R.R. }} \tilde{g}(\vec{K}) \int f(\vec{x}) e^{i \vec{K} \cdot \vec{x}} d^{3} x .
$$

Essa integral em todo espaço pode ser separada em uma soma de integrais sobre celas

\footnotetext{
${ }^{1}$ Somas sobre a rede direta e a rede recíproca são respectivamente identificadas por R.D. e R.R.

${ }^{2}$ Para o conceito de rede recíproca ver a referência [49]
} 
unitárias centradas em cada vetor $\vec{R}$ da rede direta:

$$
I=\sum_{\vec{K} \in \text { R.R. }} \tilde{g}(\vec{K}) \sum_{\vec{R} \in \text { R.D. }} \int_{\Omega_{\vec{R}}} f(\vec{x}) e^{i \vec{K} \cdot \vec{x}} d^{3} x
$$

onde denotamos por $\Omega_{\vec{R}}$ a cela unitária centrada no vetor $\vec{R}$. Podemos fazer nessa integral a substituição de variáveis $\vec{x}=\vec{R}+\vec{r}$ e ficar com:

$$
I=\sum_{\vec{K} \in \text { R.R. }} \sum_{\vec{R} \in \text { R.D. }} \tilde{g}(\vec{K}) \int_{\Omega} e^{i \vec{K} \cdot(\vec{r}+\vec{R})} f(\vec{R}+\vec{r}) d^{3} r
$$

Vamos agora usar a propriedade $e^{3} e^{i \vec{K} \cdot \vec{R}}=1$ e o fato de que estamos considerando $f(\vec{x})$ aproximadamente constante dentro de uma cela unitária. Uma vez que $\vec{r}$ varia apenas dentro da cela unitária, vamos fazer a aproximação:

$$
f(\vec{R}+\vec{r}) \cong f(\vec{R})+\vec{r} \cdot \nabla f(\vec{R})
$$

Substituindo essa aproximação na equação (A.3), temos:

$$
I=\sum_{\vec{K} \in \text { R.R. }} \sum_{\vec{R} \in \text { R.D. }} \tilde{g}(\vec{K}) \int_{\Omega} e^{i \vec{K} \cdot \vec{r}} d^{3} r f(\vec{R})+\sum_{\vec{K} \in \text { R.R. }} \sum_{\vec{R} \in \text { R.D. }} \tilde{g}(\vec{K}) \int_{\Omega} e^{i \vec{K} \cdot \vec{r}} \vec{r} d^{3} r \cdot \nabla f(\vec{R}) .
$$

Reidentificando a função $g(\vec{r})$ através da expansão da equação (A.2), temos:

$$
I=\int_{\Omega} g(\vec{r}) d^{3} r \sum_{\vec{R} \in \text { R.D. }} f(\vec{R})+\int_{\Omega} \vec{r} g(\vec{r}) d^{3} r \cdot \sum_{\vec{R} \in \text { R.D. }} \nabla f(\vec{R}) .
$$

O termo que traz o gradiente de $f(\vec{R})$ é uma estimativa do erro cometido ao se aproximar a integral $I$ pela expressão:

$$
I=\int_{\Omega} g(\vec{r}) d^{3} r \sum_{\vec{R} \in \text { R.D. }} f(\vec{R}) .
$$

Se considerarmos que a escala de distâncias em que variações apreciáveis de $f(\vec{x})$ acontecem são ordens de grandeza maiores que o parâmetro de rede, podemos ficar com a expressão (A.7) e ainda usar aproximação:

$$
\sum_{\vec{R} \in \text { R.D. }} f(\vec{R}) \Omega \cong \int f(\vec{x}) d^{3} x
$$

\footnotetext{
${ }^{3}$ Válida sempre que $\vec{K}$ pertence à rede recíproca e $\vec{R}$ à rede direta.
} 
ficando com:

$$
I=\int f(\vec{x}) g(\vec{x}) d^{3} x \cong \frac{1}{\Omega} \int_{\Omega} g(\vec{r}) d^{3} r \int f(\vec{x}) d^{3} x
$$

\section{A.1 Cálculos dos elementos de matriz da Eq.(2.22)}

Na dedução da equação 2.24 da seção 2.4, encontramos 3 elementos de matriz que envolvem integrais do tipo da equação A.1:

$$
\begin{aligned}
& \left\langle m\left|F_{m}(\vec{x}) F_{n}(\vec{x})\right| n\right\rangle \\
& \left\langle m\left|F_{m}(\vec{x}) \phi(\vec{x}) F_{n}(\vec{x})\right| n\right\rangle \\
& \left\langle m\left|F_{m}(\vec{x}) \mathcal{H} F_{n}(\vec{x})\right| n\right\rangle
\end{aligned}
$$

onde $\mathcal{H}=-\frac{p^{2}}{2 m}+V(\vec{x})$. Os dois primeiros são facilmente convertidos em integrais do tipo da equação A.1. Para A.8, temos:

$$
\left\langle m\left|F_{m}(\vec{x}) F_{n}(\vec{x})\right| n\right\rangle=\int u_{m}^{\mathbf{0}}(\vec{x}) F_{m}(\vec{x}) F_{n}(\vec{x}) u_{n}^{0}(\vec{x}) d^{3} x
$$

Aplicando o resultado A.7, temos:

$$
\begin{aligned}
\left\langle m\left|F_{m}(\vec{x}) F_{n}(\vec{x})\right| n\right\rangle & =\frac{1}{\Omega} \int_{\Omega} u_{m}^{\mathbf{0}}(\vec{x}) u_{n}^{\mathbf{0}}(\vec{x}) d^{3} x \int F_{m}(\vec{x}) F_{n}(\vec{x}) d^{3} x \\
& =\frac{\delta_{m n}}{\Omega} \int F_{m}(\vec{x}) F_{n}(\vec{x}) d^{3} x
\end{aligned}
$$

Similarmente, para A.9 temos:

$$
\left.\langle m| F_{m}(\vec{x})\right) \phi(\vec{x}) F_{n}(\vec{x})|n\rangle=\int u_{m}^{0}(\vec{x}) F_{m}(\vec{x}) \phi(\vec{x}) F_{n}(\vec{x}) u_{n}^{0}(\vec{x}) d^{3} x
$$

Aplicando novamente A.7:

$$
\begin{aligned}
\left\langle m\left|F_{m}(\vec{x}) \phi(\vec{x}) F_{n}(\vec{x})\right| n\right\rangle & =\frac{1}{\Omega} \int_{\Omega} u_{m}^{0}(\vec{x}) u_{n}^{\mathbf{0}}(\vec{x}) d^{3} x \int F_{m}(\vec{x}) \phi(\vec{x}) F_{n}(\vec{x}) d^{3} x \\
& =\frac{\delta_{m n}}{\Omega} \int F_{m}(\vec{x}) \phi(\vec{x}) F_{n}(\vec{x}) d^{3} x
\end{aligned}
$$


Para o elemento de matriz A.10, é necessário antes usar o comutador da equação 2.22 para reduzí-lo à equação 2.23 :

$$
\left\langle m\left|F_{m} \mathcal{H} F_{n}\right| n\right\rangle=\left\langle m\left|F_{m} F_{n} \mathcal{H}\right| n\right\rangle-\frac{\hbar^{2}}{2 m}\left\langle m\left|F_{m} \nabla^{2} F_{n}(\vec{x})\right| n\right\rangle-i \frac{\hbar}{m}\left\langle m\left|F_{m} \nabla F_{n}(\vec{x}) \cdot \vec{p}\right| n\right\rangle
$$

Lembrando-se que $|n\rangle$ é autoestado de $\mathcal{H}$, podemos escrever a equação acima como:

$$
\begin{aligned}
\left\langle m\left|F_{m} \mathcal{H} F_{n}\right| n\right\rangle= & E_{n}^{0} \int u_{m}^{0}(\vec{x}) F_{m}(\vec{x}) F_{n}(\vec{x}) u_{n}^{\mathbf{0}}(\vec{x}) d^{3} x \\
& -\frac{\hbar^{2}}{2 m} \int u_{m}^{0}(\vec{x}) F_{m}(\vec{x}) \nabla^{2} F_{n}(\vec{x}) u_{n}^{\mathbf{0}}(\vec{x}) d^{3} x \\
& -i \frac{\hbar}{m} \int u_{m}^{\mathbf{0}}(\vec{x}) F_{m}(\vec{x}) \nabla F_{n}(\vec{x}) \cdot \vec{p} u_{n}^{0}(\vec{x}) d^{3} x
\end{aligned}
$$

Aplicando A.7 e a ortonormalidade dos $u_{m}^{\mathbf{0}}(\vec{x})$, temos:

$$
\begin{aligned}
\left\langle m\left|F_{m} \mathcal{H} F_{n}\right| n\right\rangle= & E_{n}^{0} \frac{\delta_{m n}}{\Omega} \int F_{m}(\vec{x}) F_{n}(\vec{x}) d^{3} x \\
& -\frac{\hbar^{2}}{2 m} \frac{\delta_{m n}}{\Omega} \int F_{m}(\vec{x}) \nabla^{2} F_{n}(\vec{x}) d^{3} x \\
& -i \frac{\hbar}{m} \frac{1}{\Omega} \int F_{m}(\vec{x}) \nabla F_{n}(\vec{x}) d^{3} x \cdot \int_{\Omega} u_{m}^{\mathbf{0}}(\vec{x}) \vec{p} u_{n}^{\mathbf{0}}(\vec{x}) d^{3} x
\end{aligned}
$$

Identificando os elementos de matriz $\vec{p}_{n m}=\int_{\Omega} u_{m}^{0}(\vec{x}) \vec{p} u_{n}^{\mathbf{0}}(\vec{x}) d^{3} x$ e agrupando termos semelhantes temos finalmente:

$$
\begin{gathered}
\left\langle m\left|F_{m} \mathcal{H} F_{n}\right| n\right\rangle=\frac{1}{\Omega} \int F_{m}(\vec{x})\left\{\delta_{m n}\left[E_{n}^{0} F_{n}(\vec{x})-\frac{\hbar^{2}}{2 m} \nabla^{2} F_{n}(\vec{x})\right]\right. \\
\left.-i \frac{\hbar \vec{p}_{n m}}{m} \cdot \nabla F_{n}(\vec{x})\right\} d^{3} x
\end{gathered}
$$

